\title{
ASSESSMENT OF THE GEOTHERMAL RESOURCES \\ OF KANSAS
}

Volume II - APPENDICES

Section 5

ASO7- $99=727204$

NOTICE

PORTIONS OF THIS REPORT ARE ILLEGIBLE. It has been reproduced from the best available copy to permit the broadest possible availability.

MN ONLY 


\section{DISCLAIMER}

This report was prepared as an account of work sponsored by an agency of the United States Government. Neither the United States Government nor any agency Thereof, nor any of their employees, makes any warranty, express or implied, or assumes any legal liability or responsibility for the accuracy, completeness, or usefulness of any information, apparatus, product, or process disclosed, or represents that its use would not infringe privately owned rights. Reference herein to any specific commercial product, process, or service by trade name, trademark, manufacturer, or otherwise does not necessarily constitute or imply its endorsement, recommendation, or favoring by the United States Government or any agency thereof. The views and opinions of authors expressed herein do not necessarily state or reflect those of the United States Government or any agency thereof. 


\section{DISCLAIMER}

Portions of this document may be illegible in electronic image products. Images are produced from the best available original document. 



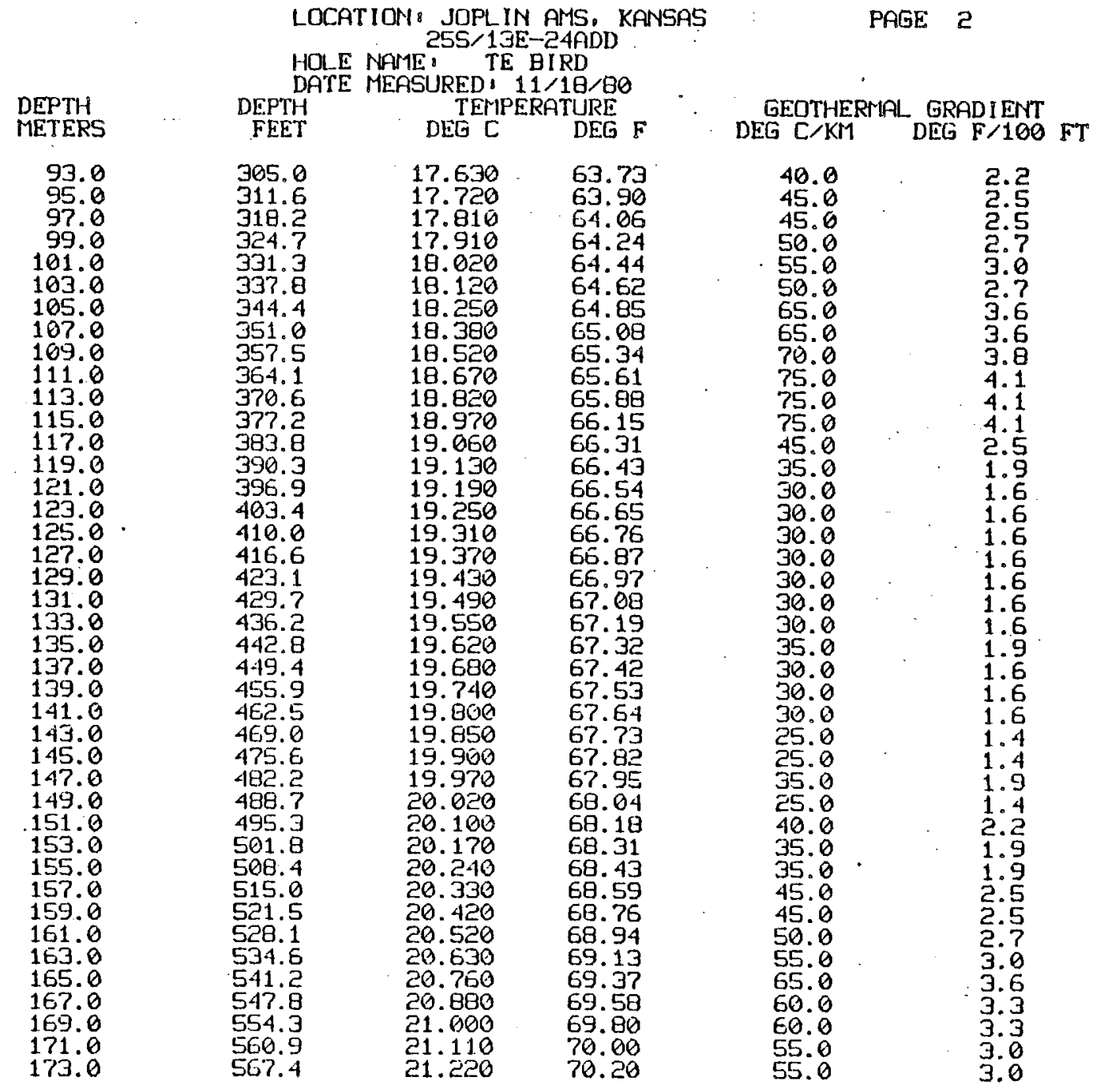




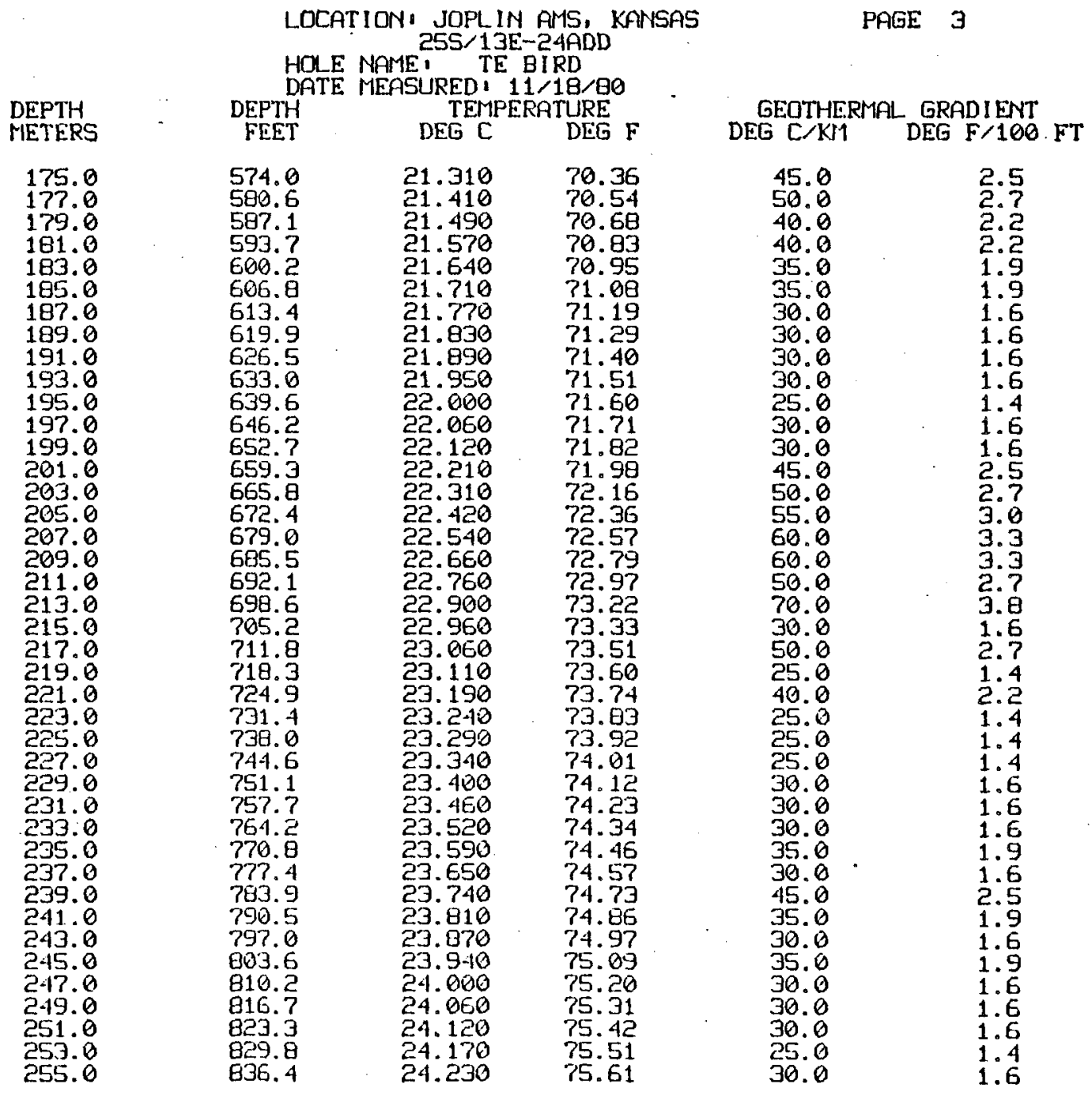




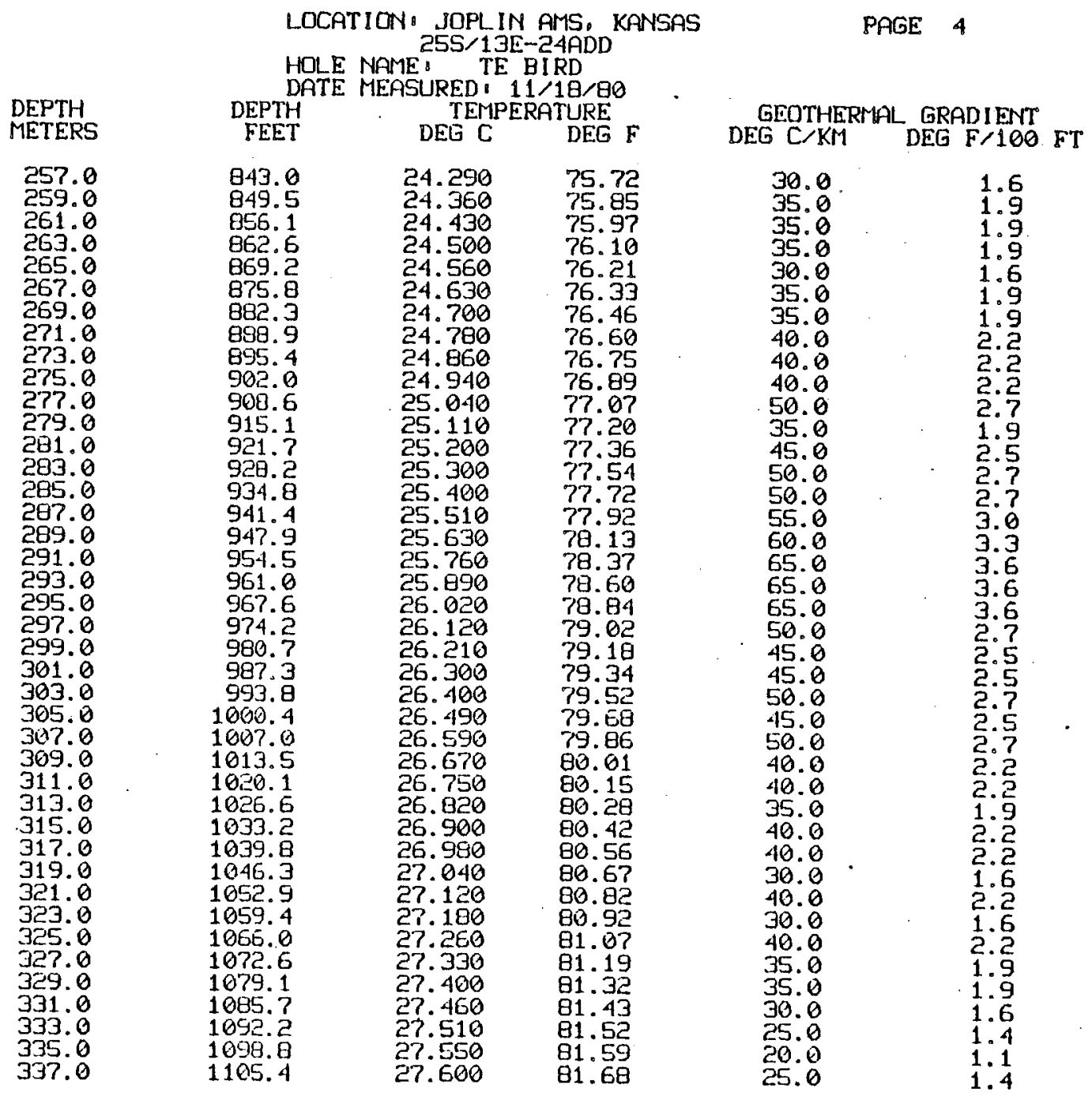


LOCATION J JOPLIN AMS,
2SS, $13 E-24 A D D$
HOLE NAME T TE BIRD
DATE MEASURED I $11 / 18 / 80$

DEPTH
METERS DEPTH

TEMPERATURE
DEG C DEG F

1111.9

339.0

27.660

81. 79

27.740

27.930

1125.0

345.0

347.0

1131.6

1139.2

1144.7

351.0

353.0

355.0

$35 ? .0$
359.0

361.0

363.0

365.0

369.0

371.0

375.0

377.0

379.0

383.0

385.0

387.0

405.0

407.0

409.0

411.0

415.0

417.0

419.0

421.0

425.0

427.8

431.0
433.0

1157.8

1164.4

1171.0

$117 ? .5$

1190.6

1197.2

1203.8

1210.3

1223.4

1230.0

1236.6

1243.1

1249.7

1256.2

20.010

28. 120

28. 240

28.340

28.430

20.600

28. 680

28.790

29.040

29.170

29.400

29.500

$29.6 B 0$

29.780

30.010

30.130

30.250

1269.4

1328.4

1335.0

134.5

1348.1

31.140

82.09

82. 26

ㄹ․ 42

92.62
82.63

63.01

63.1?

83. 32

83. 49

83.62

84.02

84.02
84.27

84.51

84.72

84.92

85. 10

85. 26

85.42

85.60

85.78
86.02

86. 02
86.23

86.45

88.05

31.370

83.29

88.47

31.550

88.47
88.79

88.93

31.750

31.880

1367.8

1374.3

1380.9

$138 ? .4$

1394.

1400.6

32.040

89. 15

89.30

89.53

89.6

32.130

32.370

32.510

1407.

$1413 . ?$

32.650
32.760

90.03
90.27

90.27

90.77

90.77
90.97

PAGE

$5:$

GEOIHERIAL GRADIENT
DEG C $/ K M$

$\begin{array}{ll}30.0 & 1.6 \\ 40.0 & 2.2 \\ 45.0 & 2.5 \\ 45.0 & 2.5 \\ 45.0 & 2.5 \\ 55.0 & 3.0 \\ 60.0 & 3.3 \\ 50.0 & 2.7 \\ 45.0 & 2.5 \\ 40.0 & 2.2 \\ 45.0 & 2.5 \\ 40.0 & 2.2 \\ 55.0 & 3.0 \\ 55.0 & 3.0 \\ 70.0 & 3.0 \\ 65.0 & 3.6 \\ 60.0 & 3.3 \\ 55.0 & 3.0 \\ 50.0 & 2.7 \\ 45.0 & 2.5 \\ 45.0 & 2.5 \\ 50.0 & 2.7 \\ 50.0 & 2.7 \\ 65.0 & 3.6 \\ 60.0 & 3.3 \\ 60.0 & 3.3 \\ 55.0 & 3.6 \\ 65.0 & 2.7 \\ 50.0 & 4.9 \\ 90.0 & 2.2 \\ 40.0 & 3.3 \\ 60.0 & 3.6 \\ 65.0 & 2.2 \\ 40.0 & 2.2 \\ 40.0 & 3.5 \\ 45.0 & 3.6 \\ 55.0 & 3.0 \\ 65.0 & 3.0 \\ 70.0 & \\ 70.0 & 5 \\ 55.0 & \end{array}$




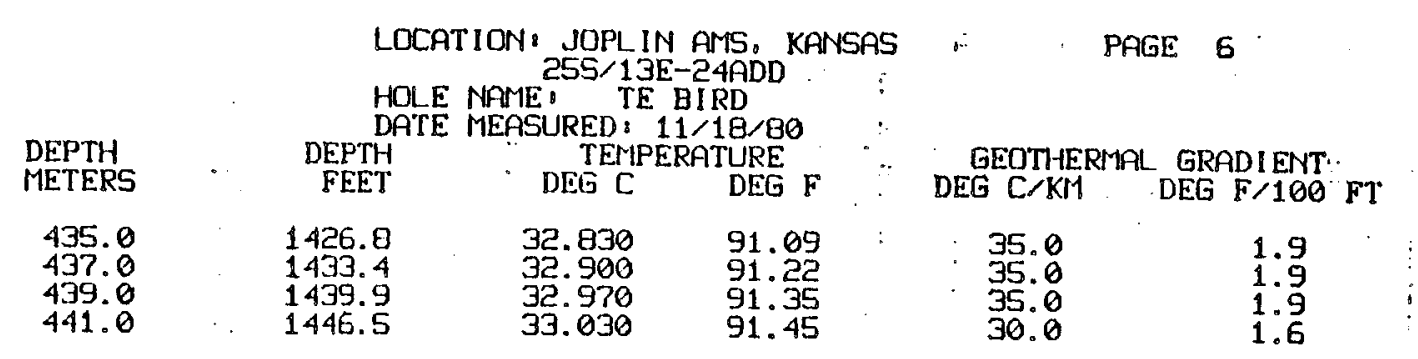




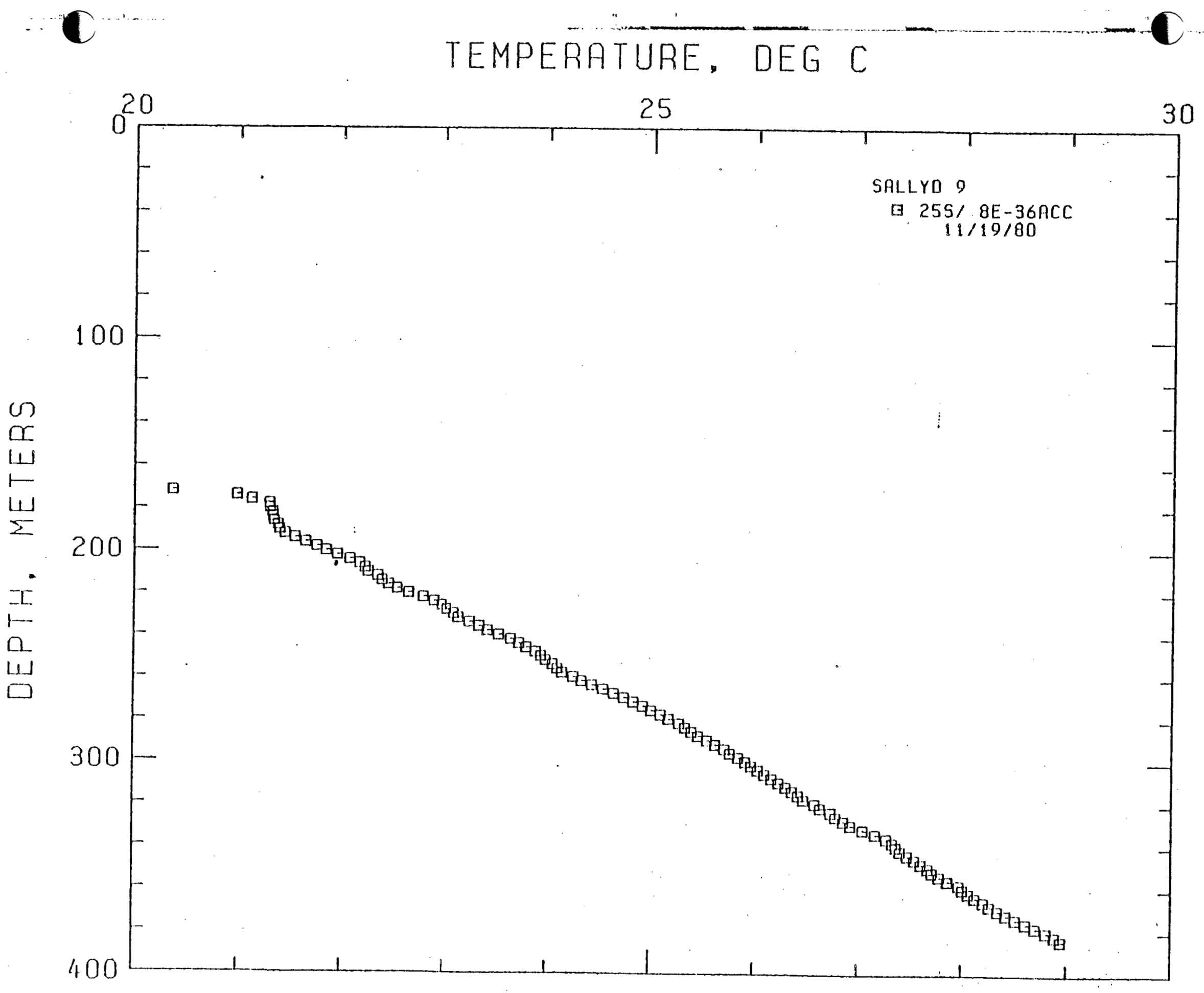



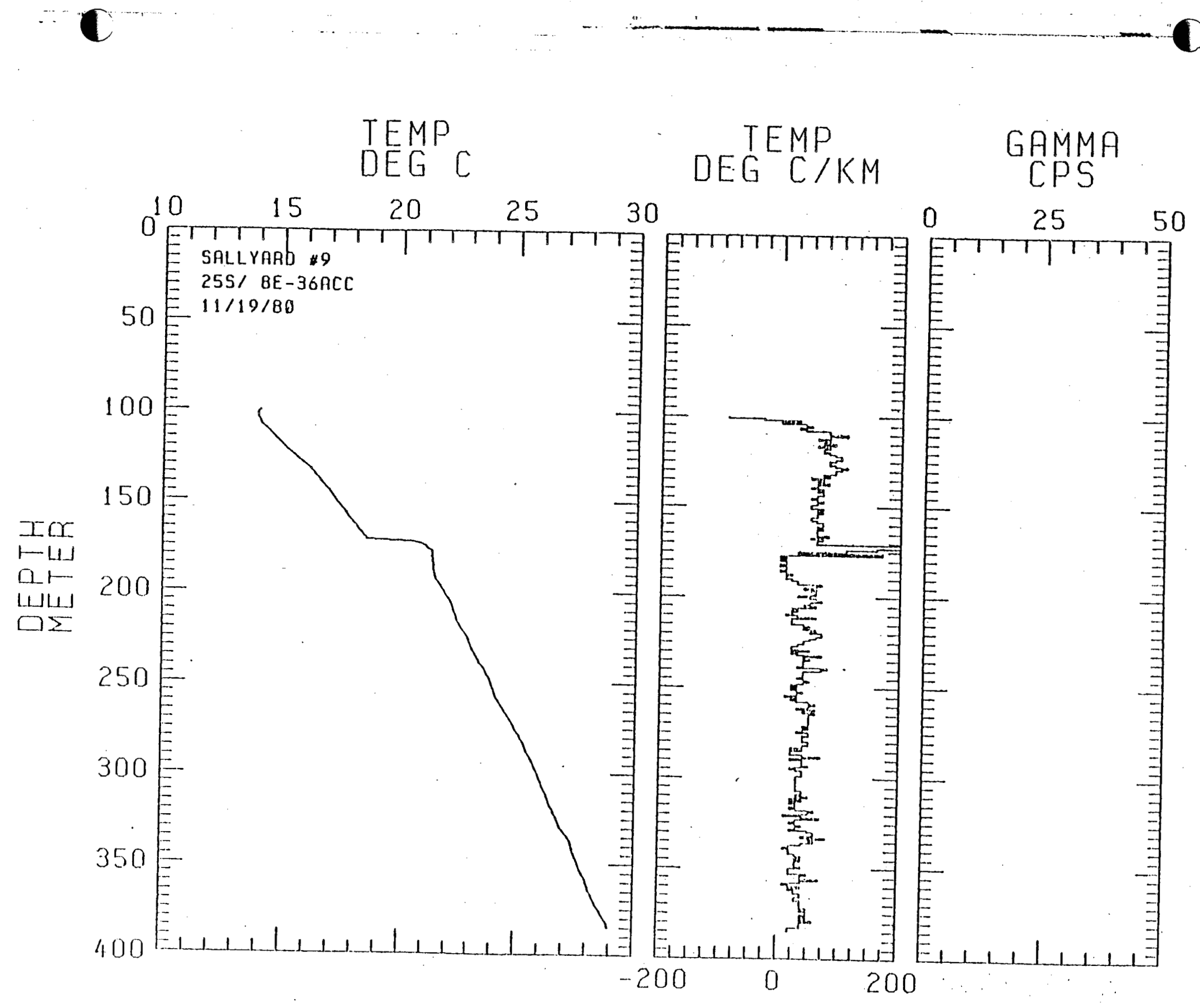

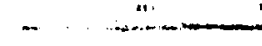

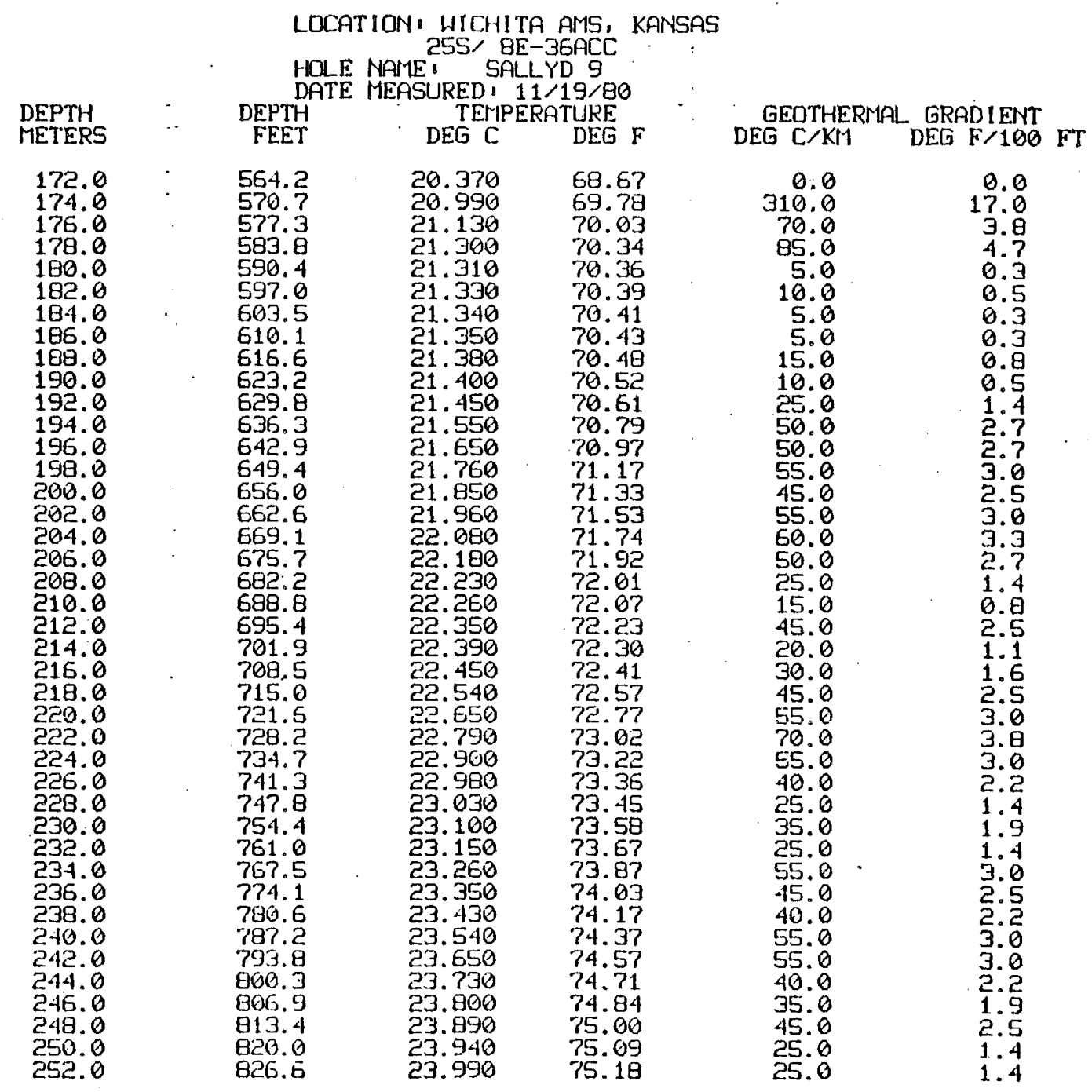


DEPTH

254.0

256.0

258.8

262.0

264.0

266.0

268.0

272.

274.0

276.0

200.0 .

282.0

284.0

288.0

290.0

292.0

294.0

298.0

300.0

302.0

304.0

308.0

310.0

314.8

316.0

118.0

320.0

324.0

326.0

330.9

334.0

DEG C
24.060
24.100
24.150
24.260
24.340
24.440
24.550
24.650
24.750
24.840
24.930
25.010
25.100
25.180
25.290
25.340
25.400
25.460
25.550
25.630
25.720
25.770
25.950
25.920
25.980
26.040
26.100
26.170
26.240
26.310
26.370
26.430
26.480
26.590
26.640
26.740
26.790
26.860
26.930
27.050
27.170

DEG $F$

GEOTHE

PAGE 2

833.1

75. 31

846.2

85c.

75.47

75.67

75.91

75.99

76.37

872.5

879.0

892. 2

898.?

911.8

918.4

925.0

931.5
938.1

944.6

$951 . ?$

957.8

964.3

970.9

984.

990.6

99?. 1

1010.2

1016.8

1023.4

1029.9

1036.5

1043.0

1049.6

1056.2

$10 E 2$ ?

1069.3

1075.8

(e⿰氵)

1095.5

27.170

76.71

76. 67

77.02

77.18

77.32

77.50

77.61

77.72

77.83

78.13

78.30

78. 39

78.53

78.66

78.75

28. 8 ?

79. 11

79.23

79.36

79.47

79.57

79.66

79.66

00.13

80.22

80.35

80.47

80.69

$$
\begin{aligned}
& 35.0 \\
& 20.0 \\
& 25.0 \\
& 55.0 \\
& 40.0 \\
& 50.0 \\
& 55.0 \\
& 50.0 \\
& 50.0 \\
& 45.0 \\
& 45.0 \\
& 40.0 \\
& 45.0 \\
& 40.0 \\
& 50.0 \\
& 30.0 \\
& 30.0 \\
& 30.0 \\
& 45.0 \\
& 40.0 \\
& 45.0 \\
& 25.0 \\
& 40.0 \\
& 35.0 \\
& 30.0 \\
& 30.0 \\
& 30.0 \\
& 35.0 \\
& 35.0 \\
& 35.0 \\
& 30.0 \\
& 30.0 \\
& 25.0 \\
& 55.0 \\
& 25.0 \\
& 50.0 \\
& 25.0 \\
& 35.0 \\
& 35.0 \\
& 60.0 \\
& 60.0 \\
&
\end{aligned}
$$

GRADIENT
DEG F/100 FT

1.9
1.1
1.4
3.9
2.2
2.7
3.0
2.7
2.7
2.5
2.5
2.2
2.5
2.2
2.7
1.6
1.6
1.6
2.5
2.2
2.5
1.4
2.2
1.9
1.6
1.6
1.6
1.9
1.9
1.9
1.6
1.6
1.4
3.0
1.4
2.7
1.4
1.9
1.9
3.3
3.3




\section{LOCATION: WICHITA AMIS, KANSAS}

HOLE NAMEI SAL/ BE-36ACC

DATE MEASURED : $11 / 19 / 90$

DEPTH
METERS DEPTH

DEG C DERPTURE $\mathrm{DEG}$

336.0

1102. 1

27.280

GEOTHERMAL GRADIENT

PAGE 3

338.

$1108.6 \quad 27.340$

1115.2

342.8

344.0

346.0

348.0

350.0

352.0

356.0

358.0

360.0

362.0

366.8

उEQ.

370.0

372.0

374.0

376.0

378.0

380.0

382.0

1121.8

$112 \mathrm{~g} 3$

1134.9

1141.4

1149.0

1154.6

1161.1

116?.?

1174.2

1180.8

118 ? 4

1193.9

1200.5

1207.0

1220.2

1226.7

1233.3

1246.4

1253.0

27. 370

27. 480

27.550

27. 610

27.680

27.790

27. 970

27.980

28.076

28.130

29.210

29.270

29.350

29. 430

29.620

28.710

28.810

28.890

91.21

Q1. 34

月1. 45

81.70

81.82

81. 90

82.02

르. $1 ?$

82. 42

82.53

62.63

르.

83.0.

63.1 ?

日3. 34

83. 52

83.68

83.06

⿶4.11

55.0
30.0
15.0
20.0
35.0
35.0
30.0
35.0
20.0
35.0
40.0
55.0
15.0
30.0
30.0
40.0
30.0
40.0
40.0
45.0
50.0
45.0
50.0
40.0
30.0

3.0

1.6

1.1

1.9

1.6

1.1

1.9

2.2

3.0

0.8

(5.8

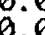

30.8

.

5.8

40.0

1. 6

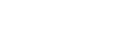

.

(1) 
TEMPERATURE, $\overline{D E G} \mathrm{C}-\mathrm{C}$

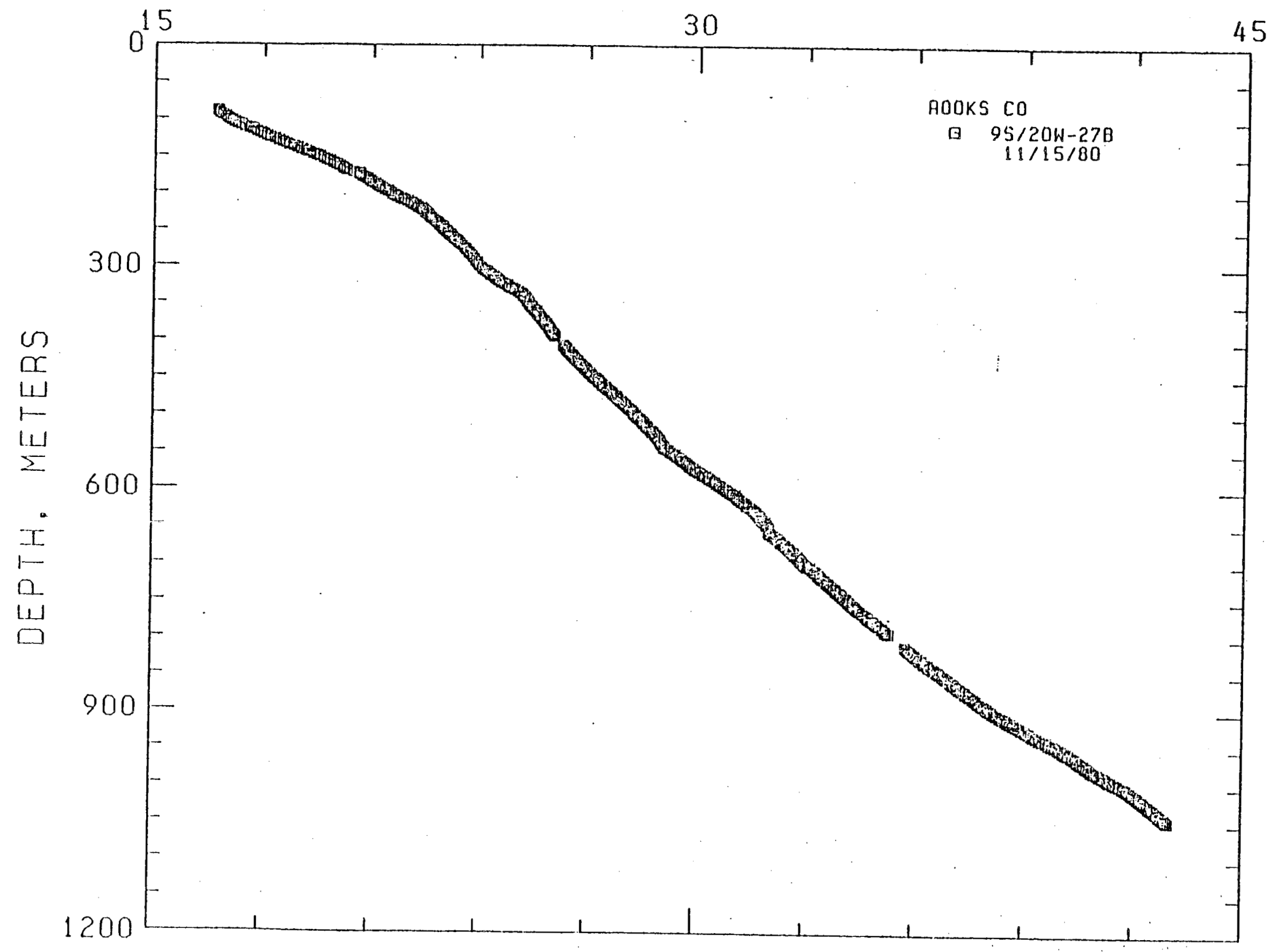




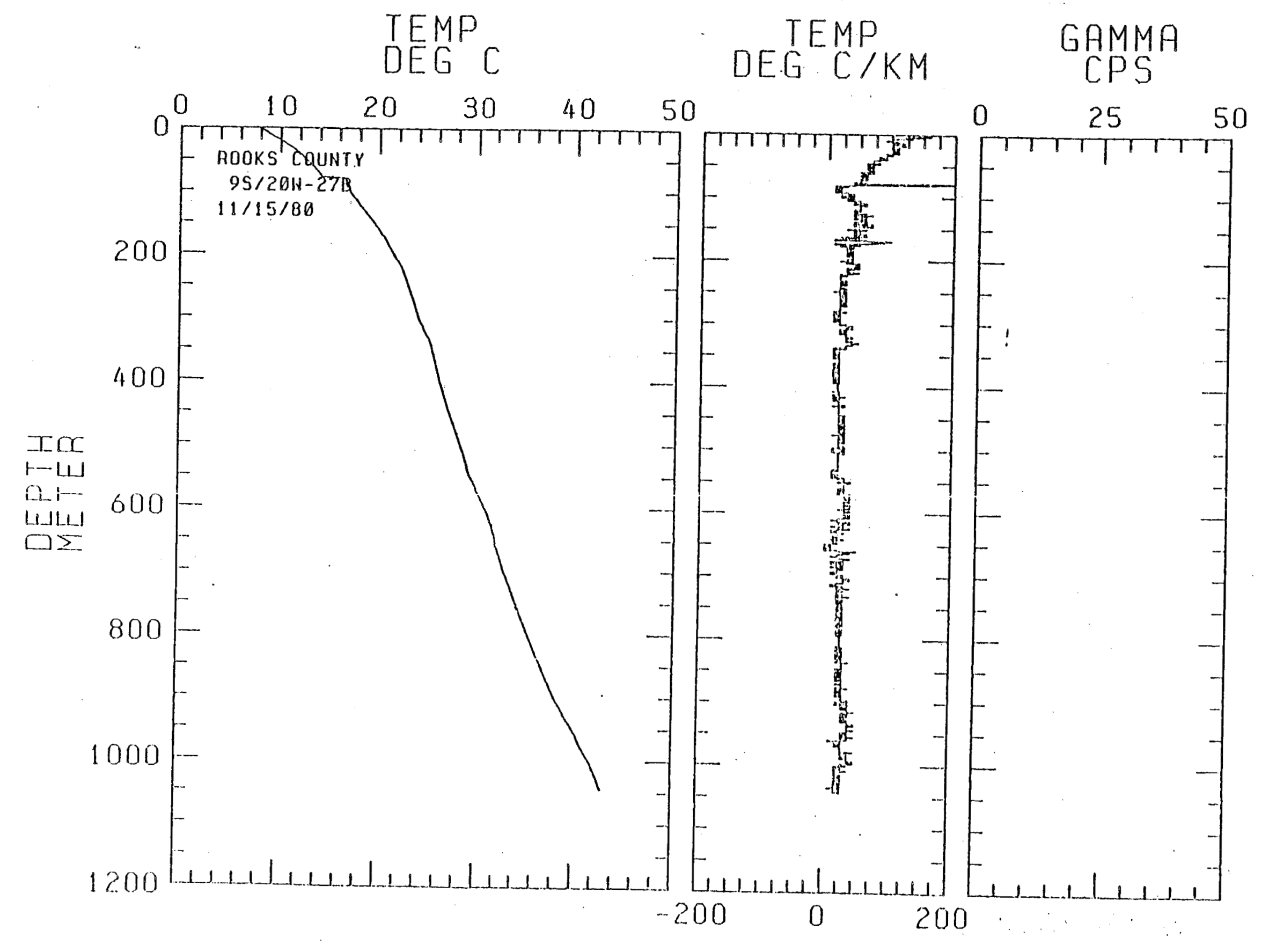




\begin{tabular}{|c|c|c|c|c|c|}
\hline $\begin{array}{l}\text { DEPTH } \\
\text { METERS }\end{array}$ & \multicolumn{3}{|c|}{ 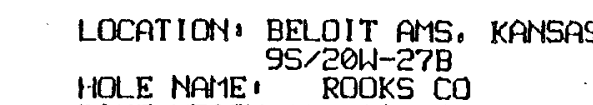 } & \multicolumn{2}{|c|}{$\begin{array}{l}\text { GEOTHERMAL GRADIENT } \\
\text { DEG } \mathrm{C} / \mathrm{KM}\end{array}$} \\
\hline & 291.9 & 16.730 & 62.11 & & \\
\hline $\begin{array}{l}91.0 \\
93.0 \\
95.9\end{array}$ & $\begin{array}{l}59.5 \\
395.0 \\
71.6\end{array}$ & $\begin{array}{l}16.720 \\
16.800 \\
16.879\end{array}$ & $\begin{array}{l}62.19 \\
62.24 \\
62.25\end{array}$ & $\begin{array}{l}20.8 \\
15: 0\end{array}$ & $\begin{array}{l}1.0 \\
0.8\end{array}$ \\
\hline $\begin{array}{l}95.0 \\
97.0 \\
99.0\end{array}$ & $\begin{array}{l}31.6 \\
318.2 \\
334 .\end{array}$ & $\begin{array}{l}16.870 \\
16.950 \\
17.910\end{array}$ & $\begin{array}{l}62.37 \\
62.51 \\
62\end{array}$ & $\begin{array}{l}35.0 \\
40.8\end{array}$ & $\begin{array}{l}1.9 \\
2.2\end{array}$ \\
\hline $\begin{array}{l}101: 0 \\
103: 0\end{array}$ & $\begin{array}{l}3<4.1 \\
331: 3 \\
332.8\end{array}$ & $\begin{array}{l}17.010 \\
17.060 \\
1730\end{array}$ & $\begin{array}{l}62.62 \\
62.71 \\
67\end{array}$ & $\begin{array}{l}30.0 \\
25.0 \\
35: 0\end{array}$ & $\begin{array}{l}1.6 \\
1.4 \\
1.4\end{array}$ \\
\hline $\begin{array}{l}105.8 \\
107.8\end{array}$ & $\begin{array}{l}33.8 \\
354: 4 \\
351.0\end{array}$ & $\begin{array}{l}17: 200 \\
17.290\end{array}$ & $\begin{array}{l}6.83 \\
6.96 \\
63\end{array}$ & $\begin{array}{l}35.8 \\
35: 0 \\
45.8\end{array}$ & $\begin{array}{l}1.9 \\
1: 9 \\
2.5\end{array}$ \\
\hline $\begin{array}{l}109.8 \\
111.8\end{array}$ & $\begin{array}{l}355.5 \\
364.1\end{array}$ & $\begin{array}{l}17.390 \\
17.490\end{array}$ & $\begin{array}{l}63.30 \\
63.48\end{array}$ & $\begin{array}{l}50.8 \\
50.0\end{array}$ & $\begin{array}{l}2.7 \\
2.7\end{array}$ \\
\hline 113.8 & $\begin{array}{l}370.6 \\
372: 5 \\
389.5\end{array}$ & $\begin{array}{l}17.610 \\
17.710\end{array}$ & $\begin{array}{l}63.70 \\
63.88 \\
64\end{array}$ & $\begin{array}{l}60.0 \\
50.8 \\
59.8\end{array}$ & $\begin{array}{l}3.9 \\
2 . ?\end{array}$ \\
\hline & $\begin{array}{l}390.3 \\
396.9\end{array}$ & $\begin{array}{l}17.910 \\
19.000\end{array}$ & $\begin{array}{l}64.24 \\
64.40\end{array}$ & $\begin{array}{l}56.0 \\
50.0 \\
45.8\end{array}$ & $\begin{array}{l}5.7 \\
2.7 \\
2.5\end{array}$ \\
\hline 3.8 & $\begin{array}{l}40.4 \\
410.8 \\
415.5\end{array}$ & $\begin{array}{l}18.090 \\
18.180\end{array}$ & $\begin{array}{l}64.56 \\
66.72\end{array}$ & $\begin{array}{l}45.0 \\
45.0 \\
69.0\end{array}$ & $\begin{array}{l}2.5 \\
2.5\end{array}$ \\
\hline & $\begin{array}{l}423.1 \\
429.7\end{array}$ & $\begin{array}{l}18.410 \\
18.510\end{array}$ & $\begin{array}{l}65.14 \\
65.32\end{array}$ & $\begin{array}{l}55.8 \\
50.8\end{array}$ & $\begin{array}{ll}3.0 \\
2.7\end{array}$ \\
\hline $\begin{array}{l}139.0 \\
135.8 \\
137.8\end{array}$ & $\begin{array}{l}-46.5 \\
442 . \mathrm{G} \\
449\end{array}$ & $\begin{array}{l}18.610 \\
18.710\end{array}$ & $\begin{array}{l}65.56 \\
65.68 \\
65.68\end{array}$ & $\begin{array}{l}50.8 \\
50.0 \\
55.0\end{array}$ & $\begin{array}{l}2 . ? \\
2 . ? \\
2 . ?\end{array}$ \\
\hline & 59 & $\begin{array}{l}18.93515 \\
19.030\end{array}$ & 65 & 55.0 & 30 \\
\hline & 9.0 & $\begin{array}{l}19.1 .10 \\
19.240\end{array}$ & 66.45 & 55.0 & 年. \\
\hline 149.0 & $\begin{array}{l}482.2 \\
4 \in 8.2\end{array}$ & $\begin{array}{l}19.360 \\
19.460\end{array}$ & $\begin{array}{l}66.85 \\
67.03\end{array}$ & $\begin{array}{l}60.0 \\
50.0\end{array}$ & $\begin{array}{l}3.9 \\
3 \\
3\end{array}$ \\
\hline & $\begin{array}{l}495.3 \\
501.8\end{array}$ & $\begin{array}{l}19.540 \\
19.640\end{array}$ & $\begin{array}{l}67.17 \\
6 ? .35\end{array}$ & $\begin{array}{l}40.0 \\
50.0 \\
50\end{array}$ & $\begin{array}{l}\text { a. } \\
2 . ? \\
2 . ?\end{array}$ \\
\hline $\begin{array}{l}155.8 \\
157.8\end{array}$ & $\begin{array}{l}500.4 \\
515.0\end{array}$ & $\begin{array}{l}19.730 \\
19.810\end{array}$ & $\begin{array}{l}67.51 \\
67.66\end{array}$ & $\begin{array}{l}45.0 \\
40.0\end{array}$ & 2. 2 \\
\hline & $\begin{array}{l}520.1 \\
534.6\end{array}$ & $\begin{array}{l}19.010 \\
20.090\end{array}$ & $\begin{array}{l}-02 \\
68.02 \\
6 \notin .16\end{array}$ & $\begin{array}{l}55.6 \\
45.6 \\
40.0\end{array}$ & \\
\hline & 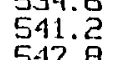 & $\begin{array}{l}20.170 \\
20.170\end{array}$ & 60.31 & 40 & \\
\hline & 554.3 & 20.280 & 5 & 35 & $\begin{array}{l}1.1 \\
1.9\end{array}$ \\
\hline
\end{tabular}




\section{- ,}

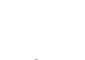

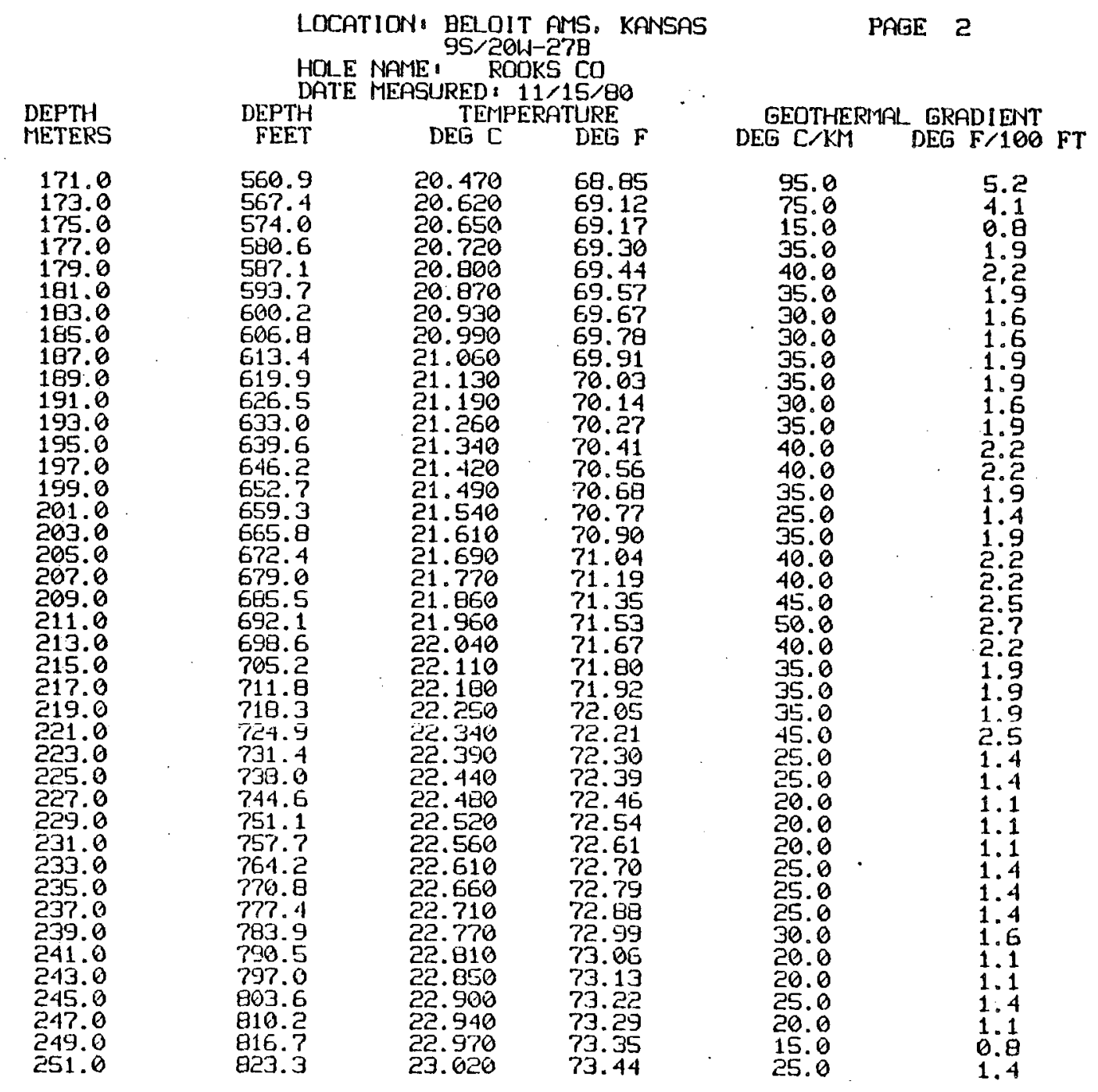




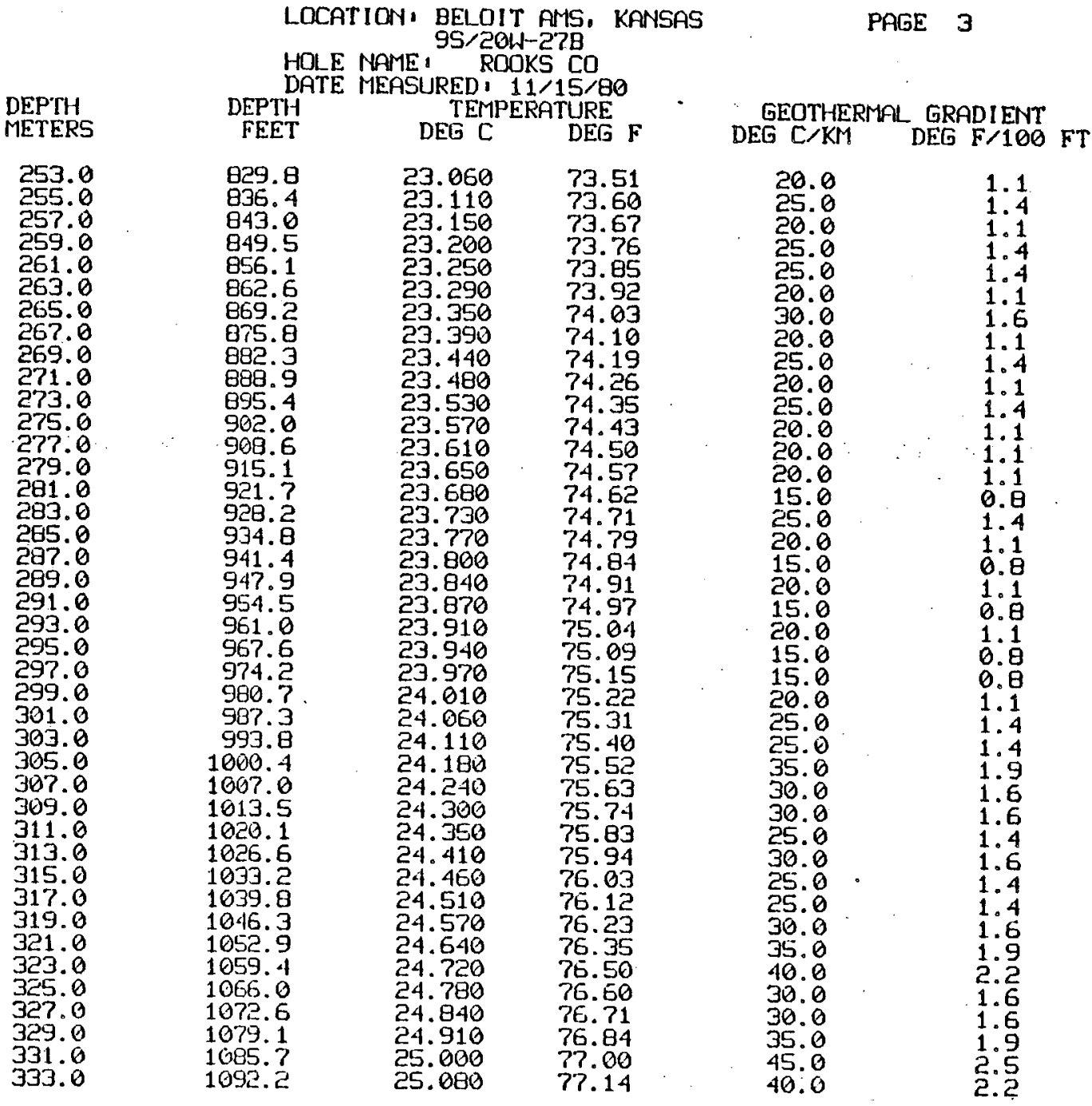


LOCATION. BELOIT AMS, KANSAS

HOLE NAME, 95/20W-27B

HOLE NAME' ROOKS CO

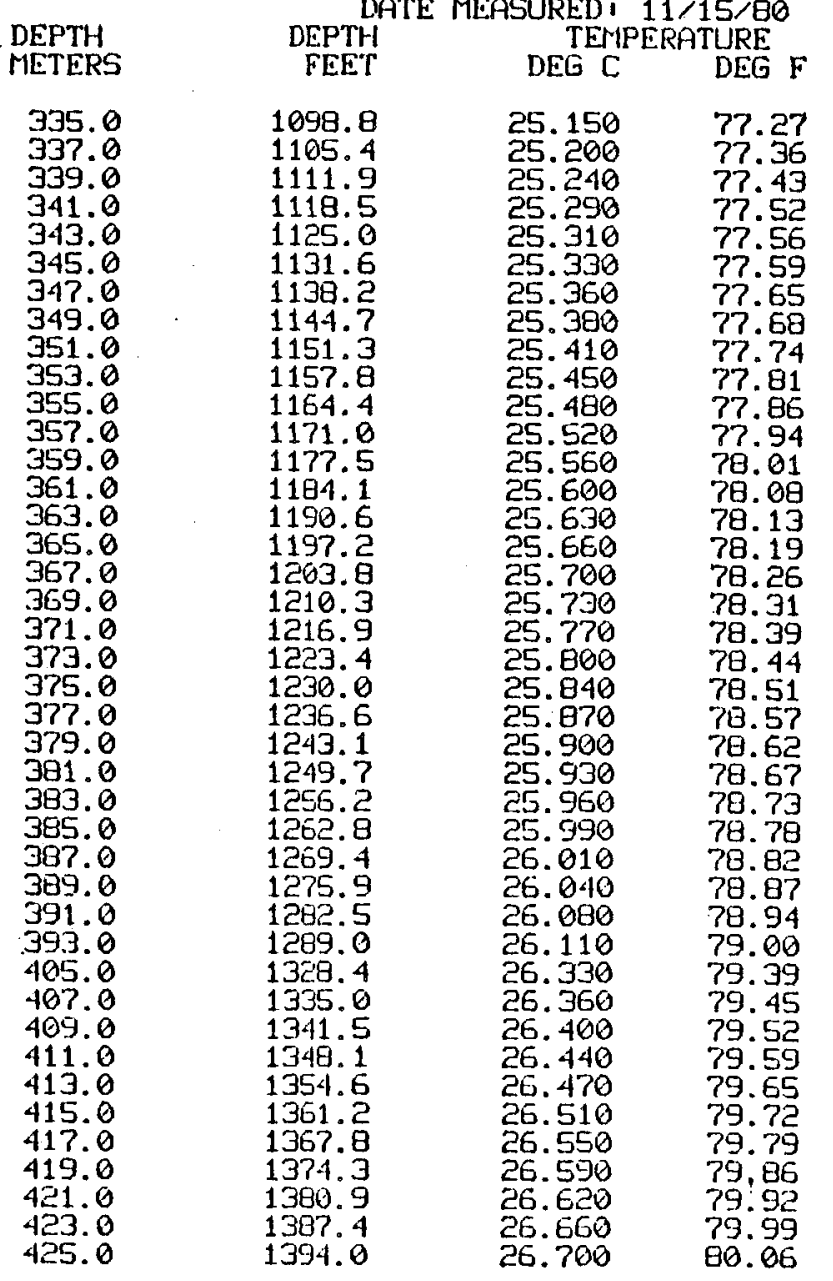

PAGE 4

GETHERIAL GRADIENT DEG $\mathrm{C} / \mathrm{KM}$ DEG F/100 FT

\begin{tabular}{|c|c|}
\hline $\begin{array}{l}35.0 \\
25.0 \\
20.0 \\
25.0 \\
10.0 \\
10.0 \\
15.0 \\
10.0 \\
15.0 \\
20.0 \\
15.0 \\
20.0 \\
20.0 \\
20.0 \\
15.0 \\
15.0 \\
20.0 \\
15.0 \\
20.0 \\
15.0 \\
20.0 \\
15.0 \\
15.0 \\
15.0 \\
15.0 \\
15.0 \\
10.0 \\
15.0 \\
20.0 \\
15.0 \\
10.0 \\
15.0 \\
20.0 \\
20.0 \\
15.0 \\
20.0 \\
20.0 \\
20.0 \\
15.0 \\
20.0 \\
20.0\end{array}$ & 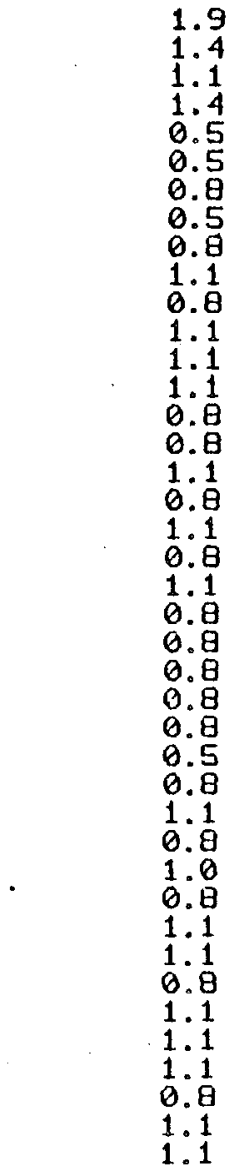 \\
\hline
\end{tabular}




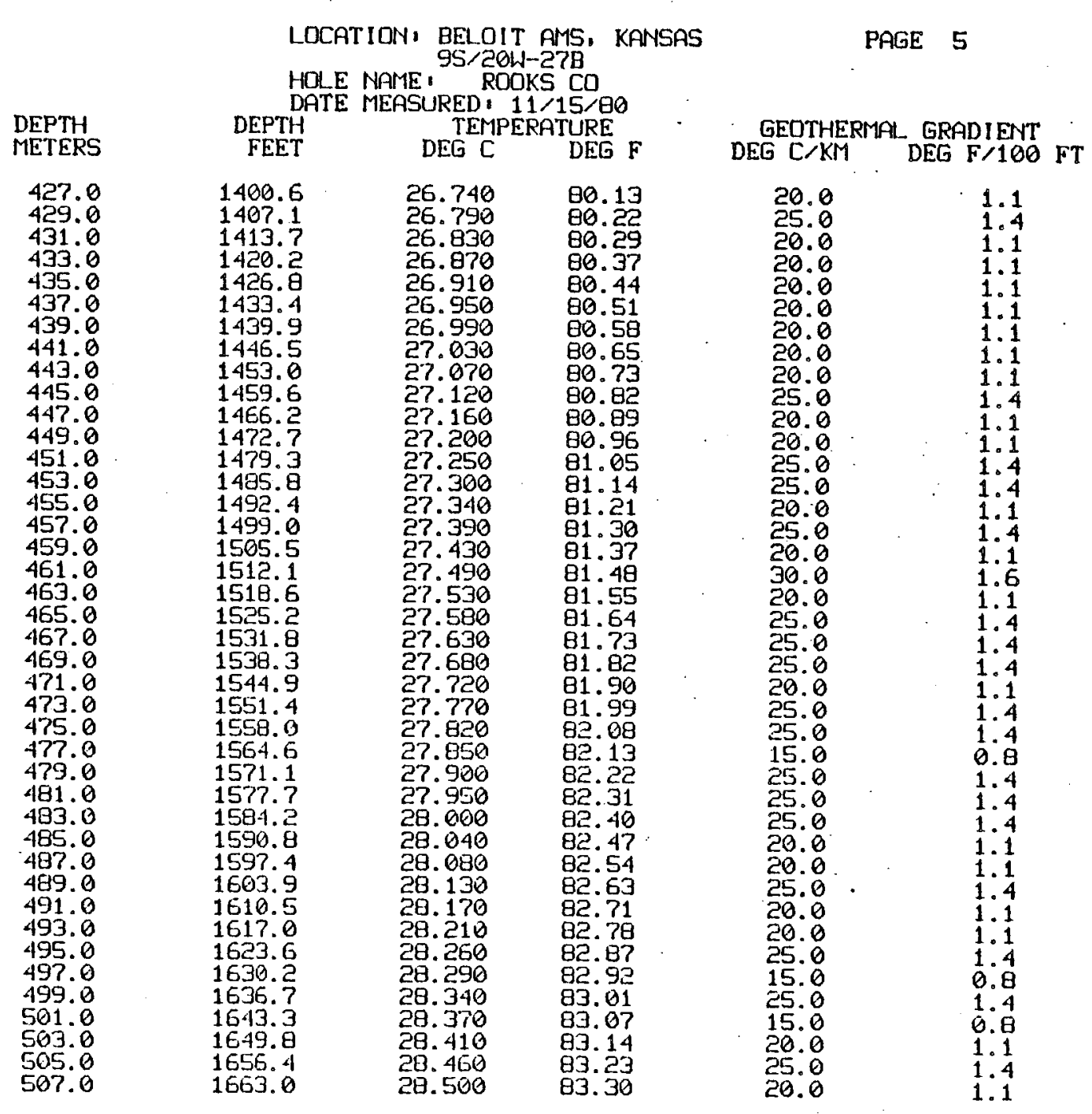




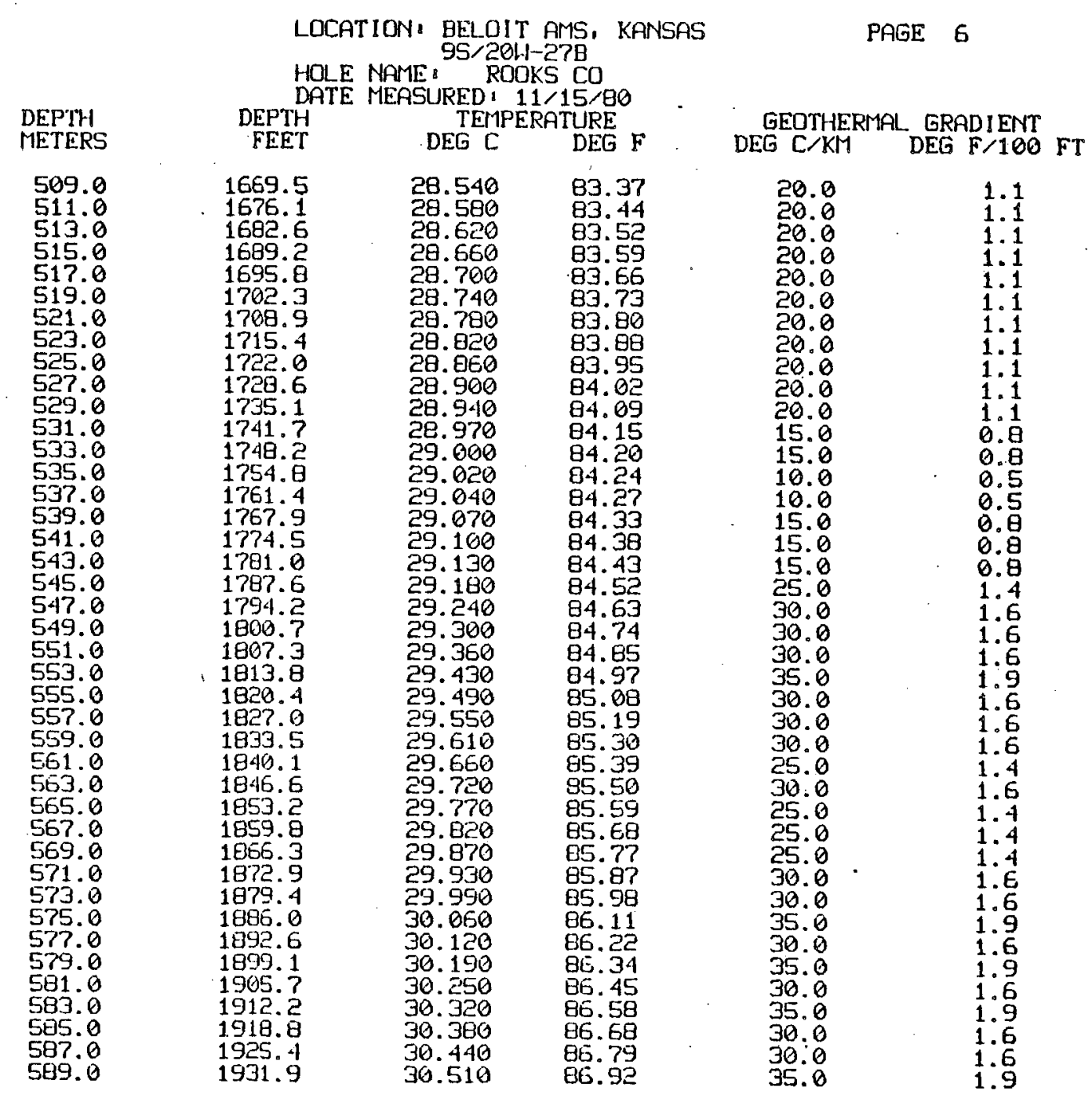




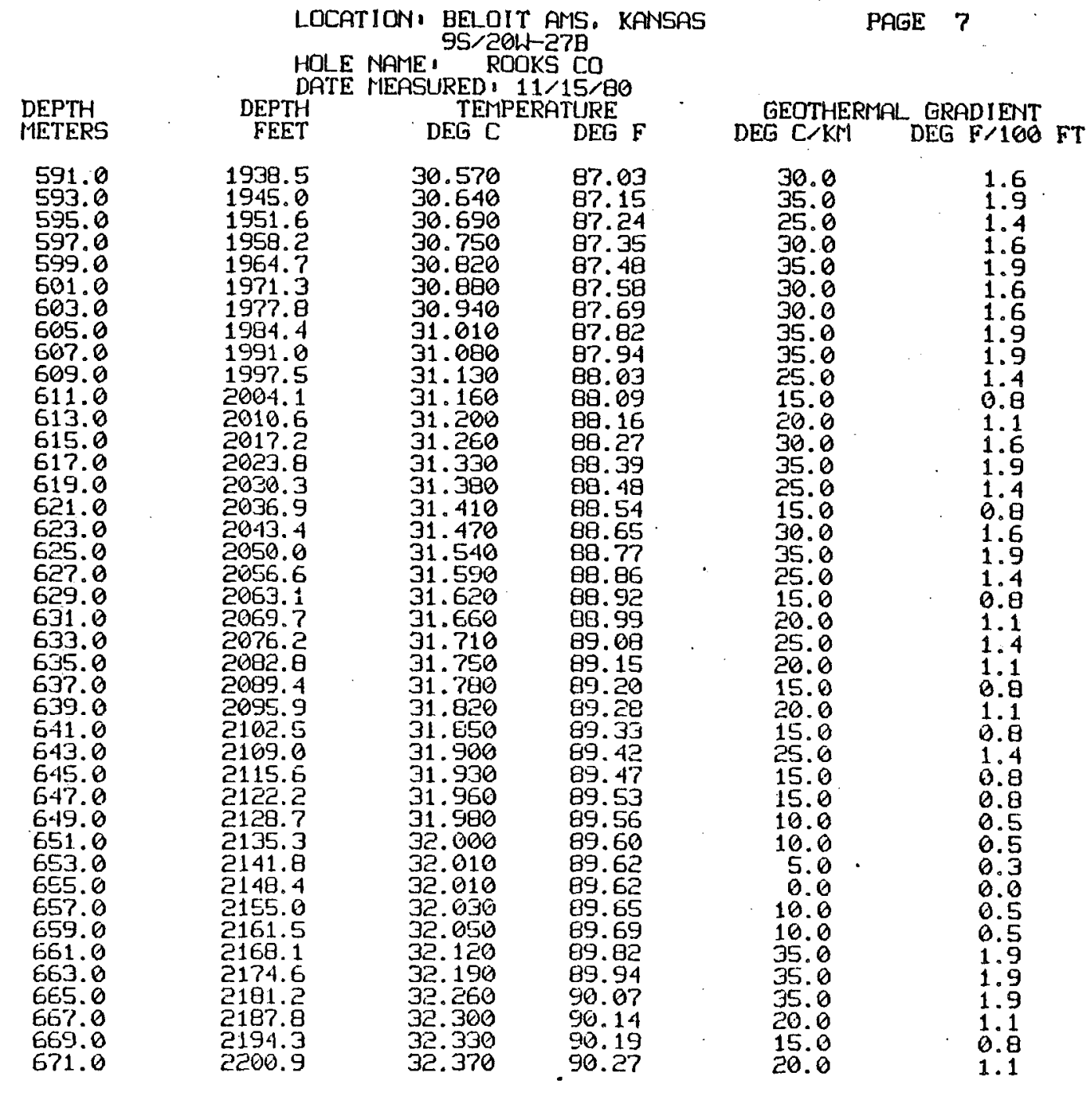




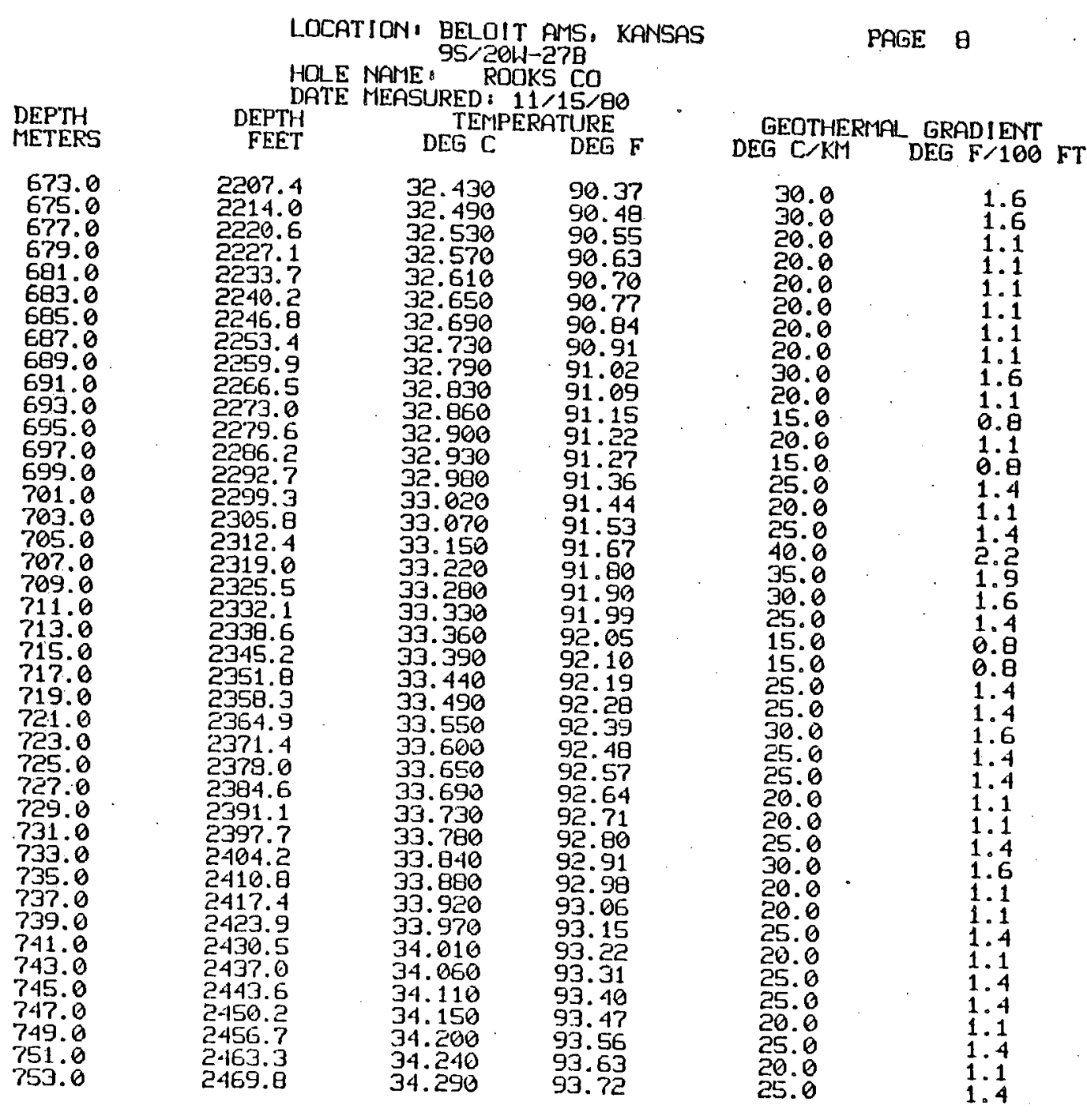




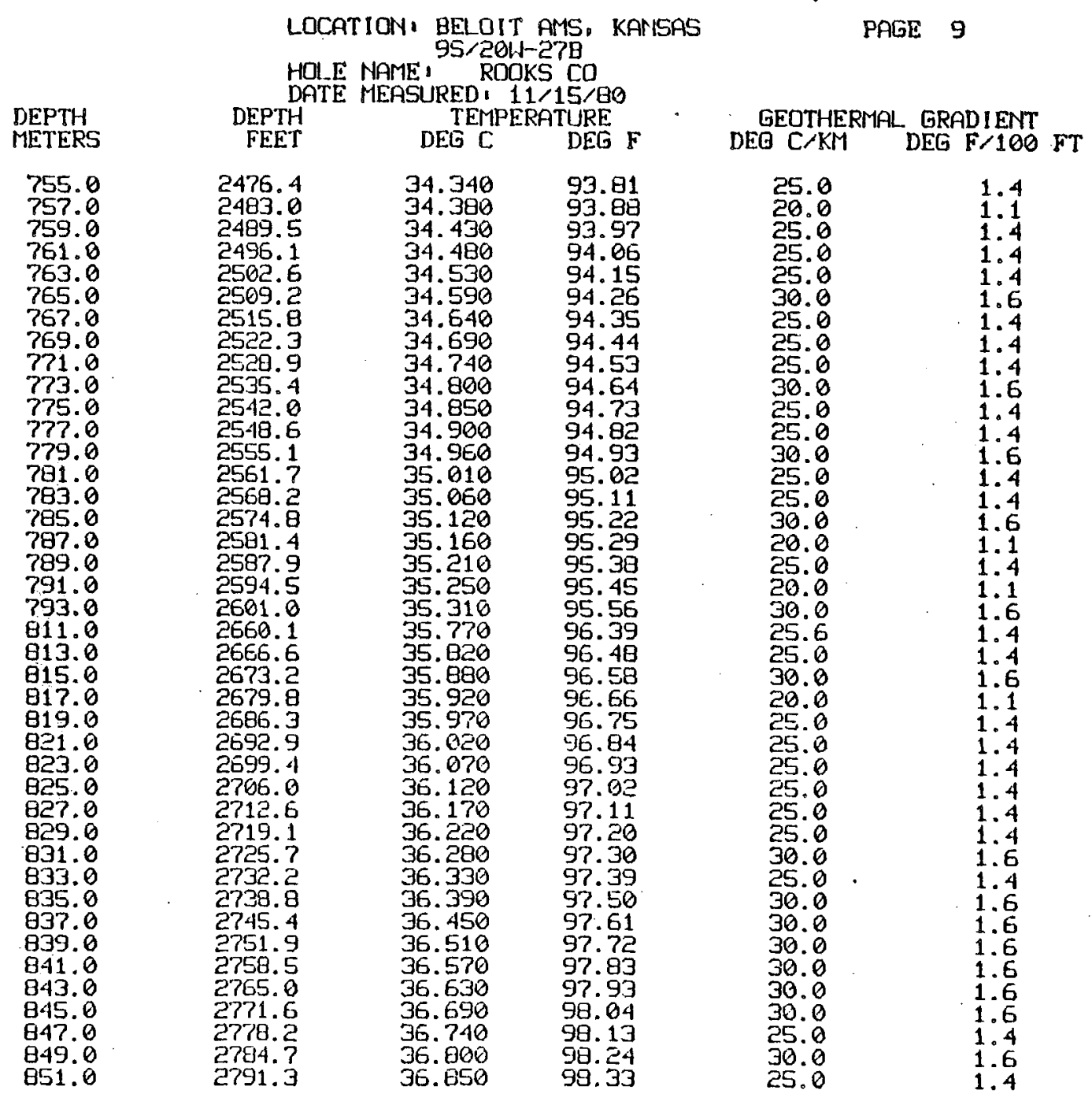




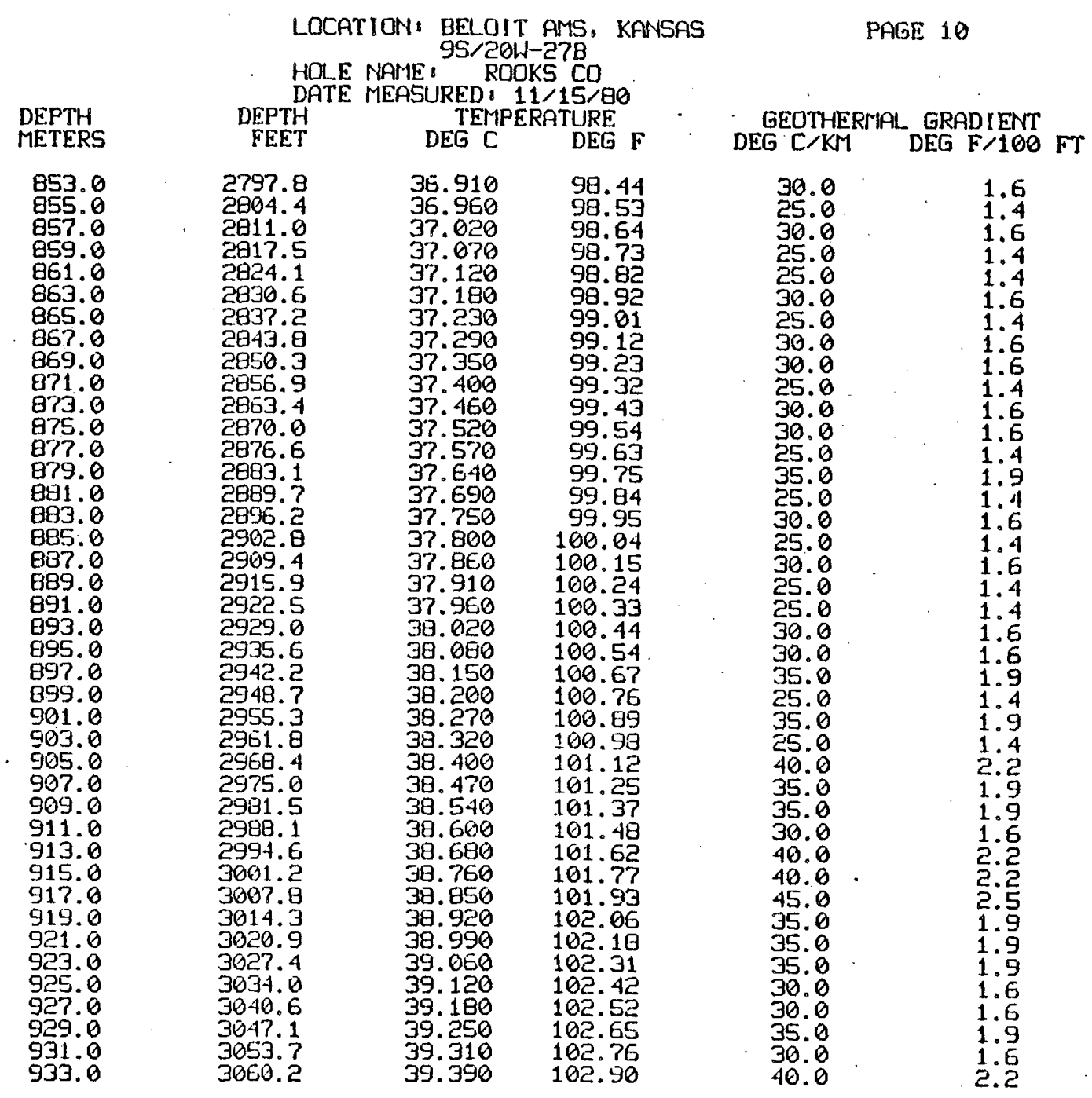




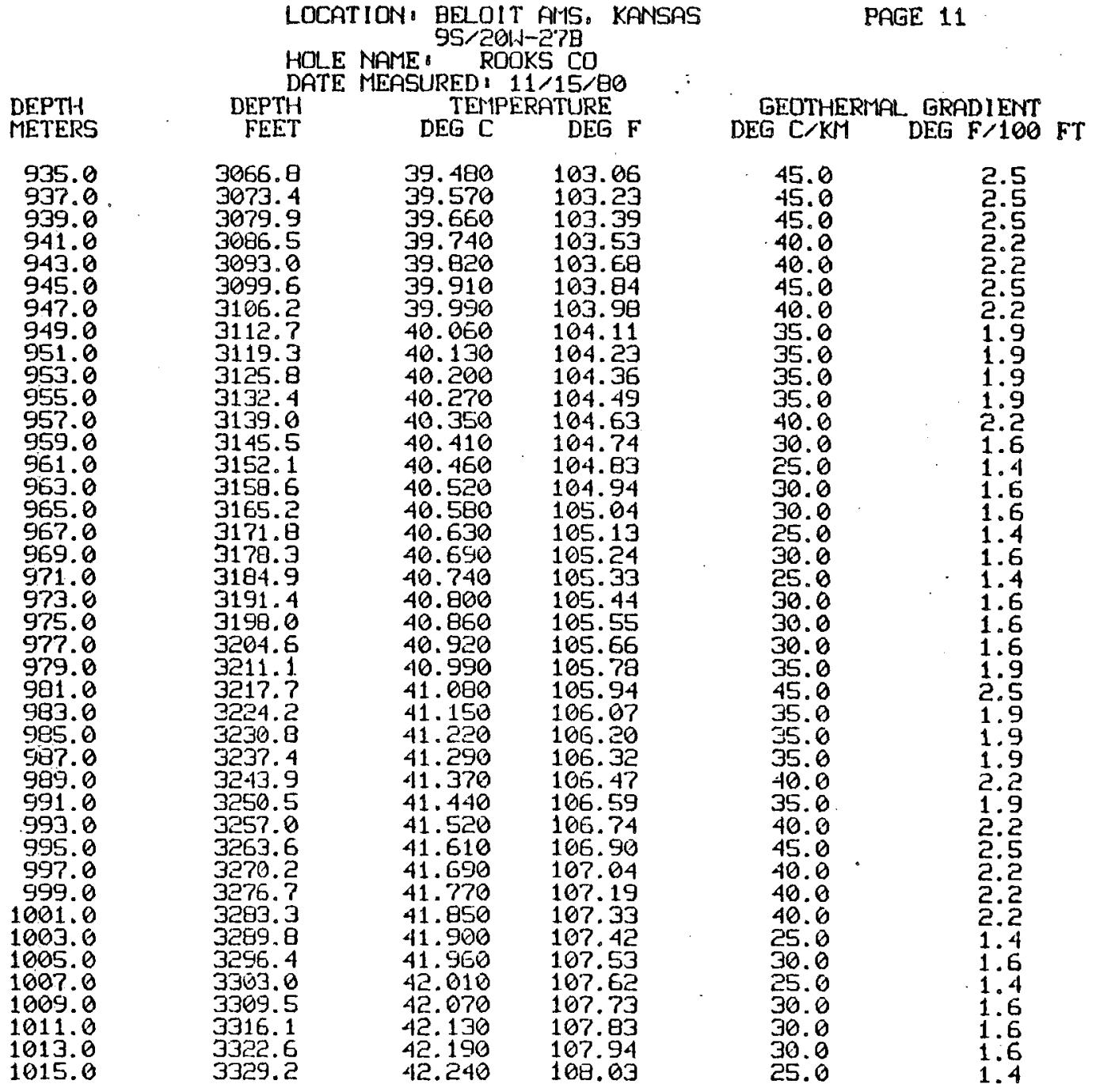




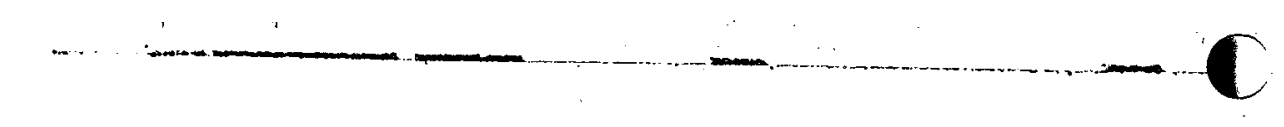

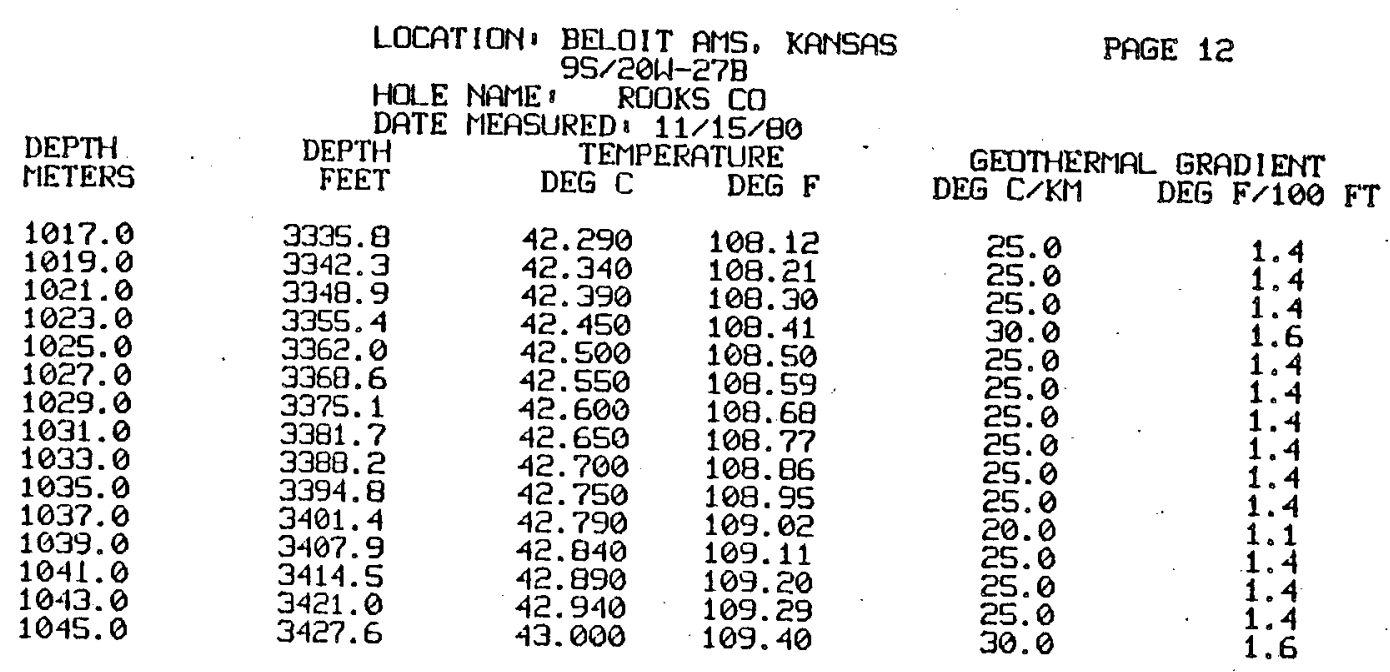




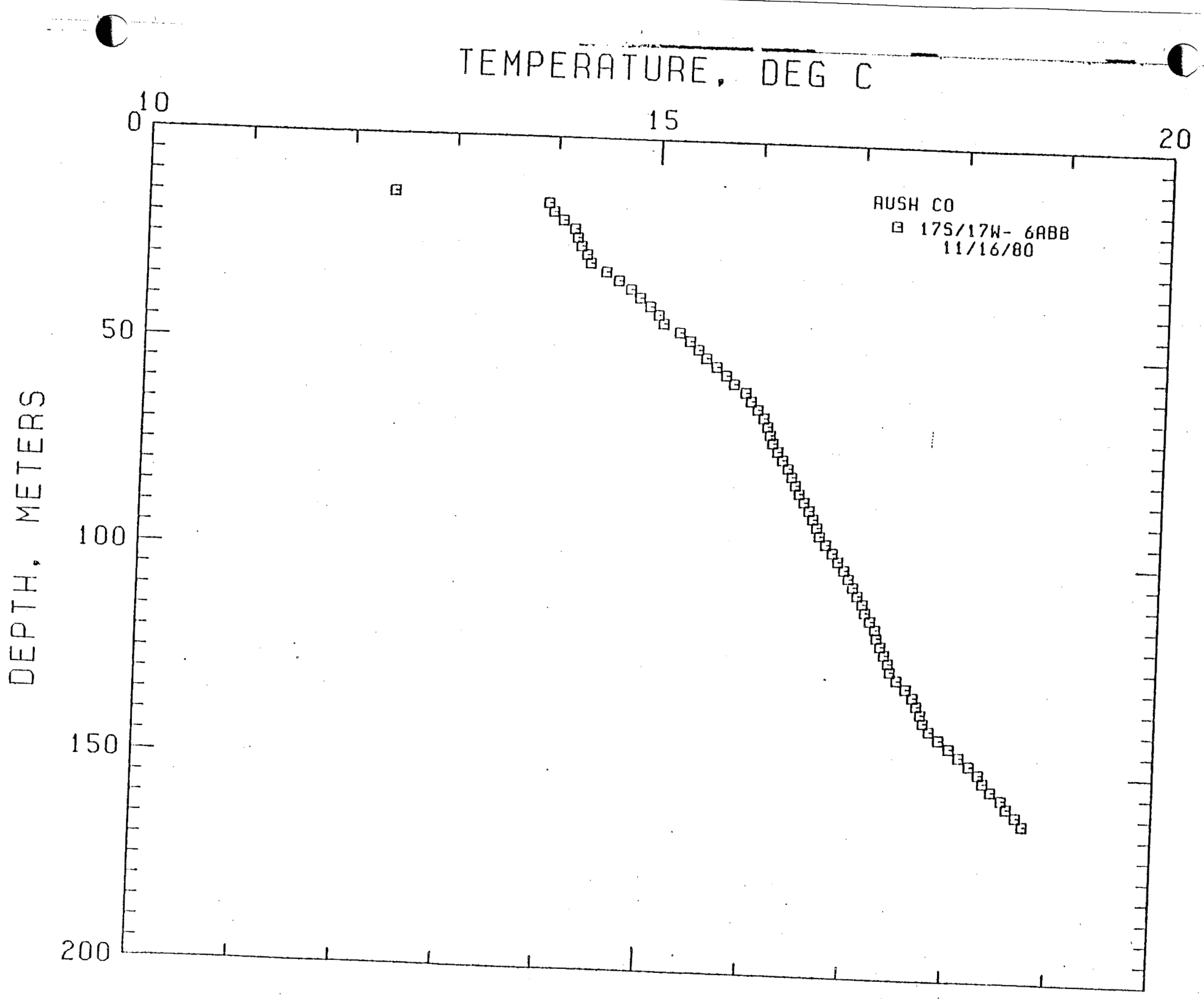




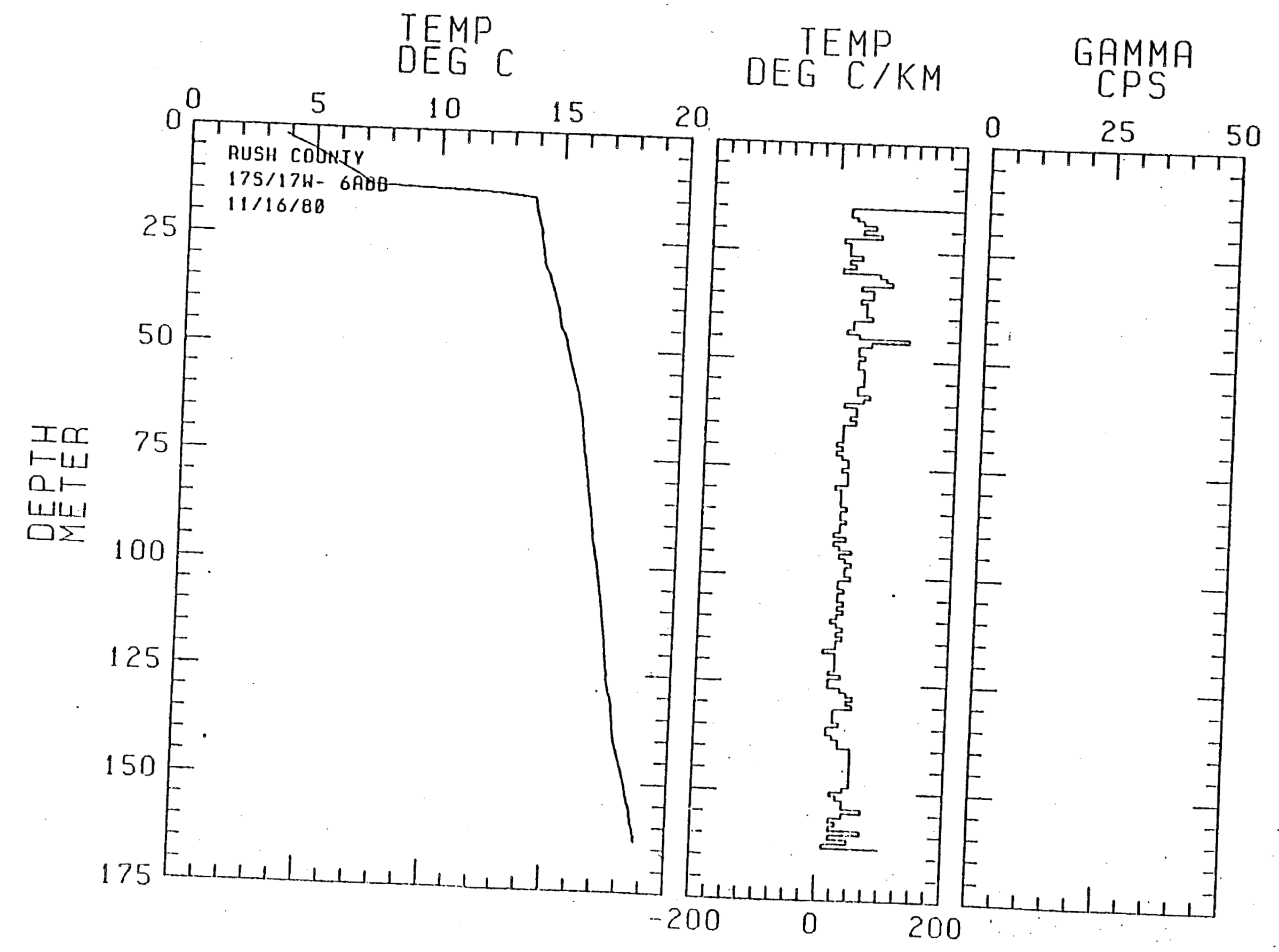




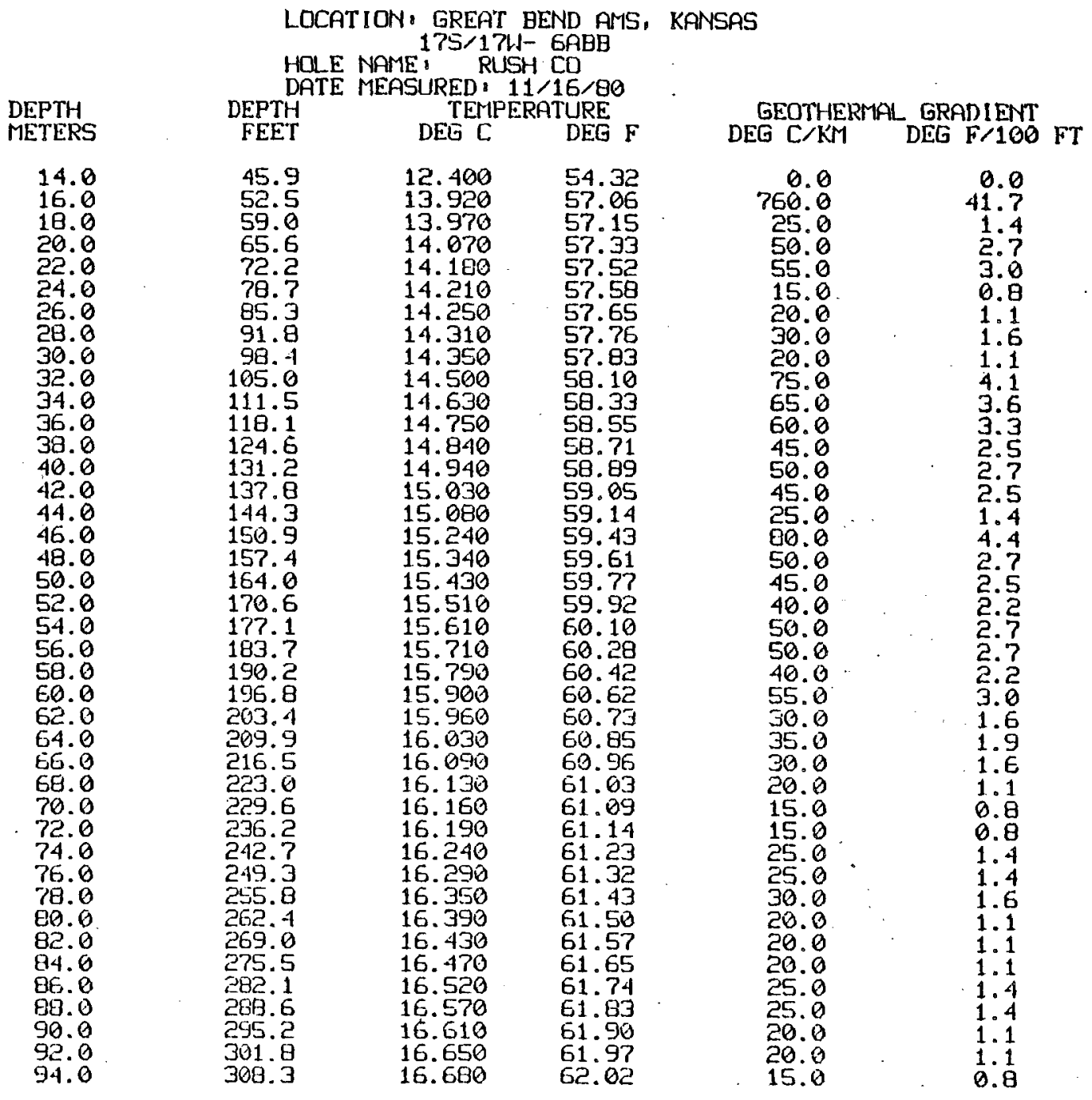




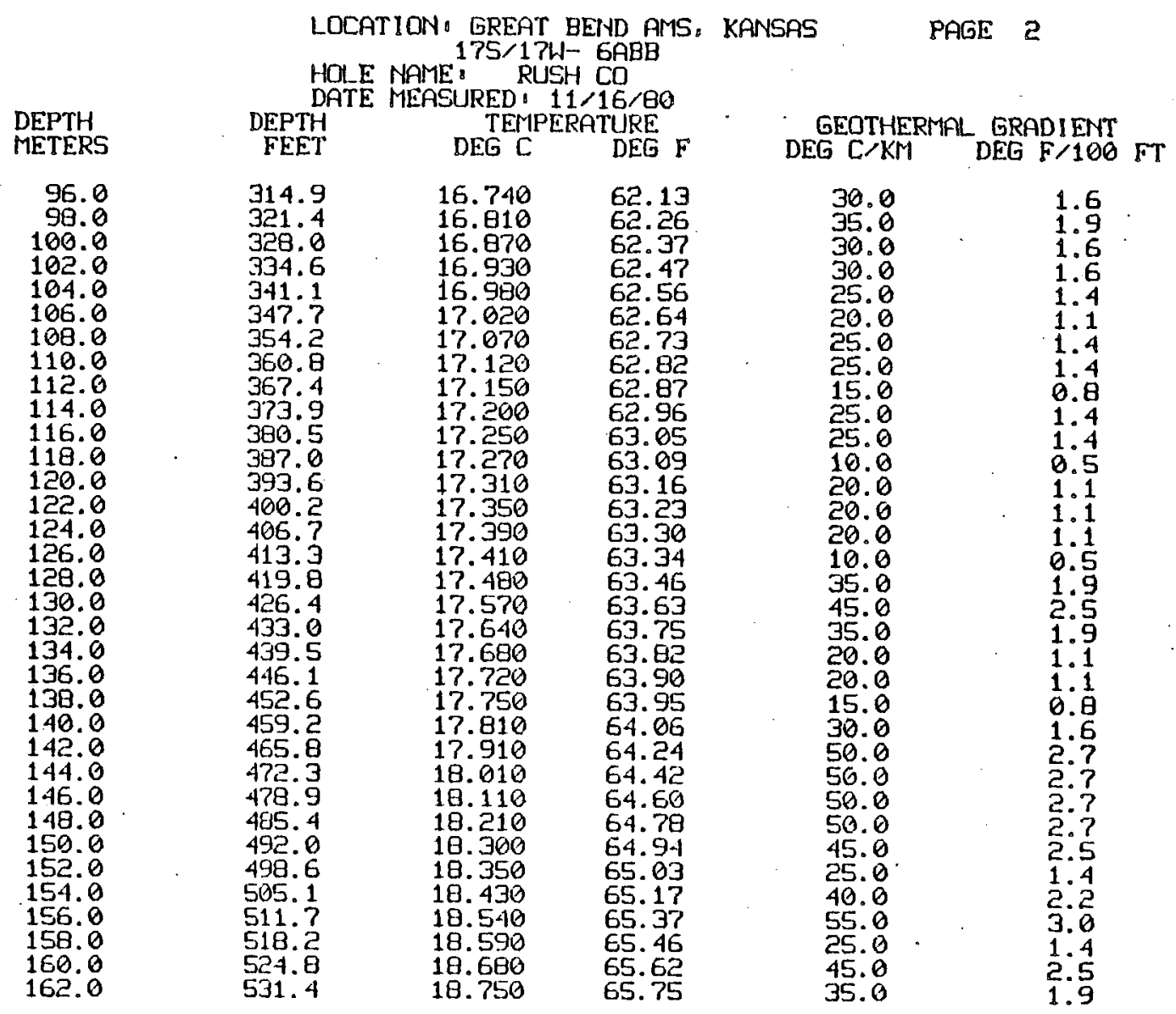




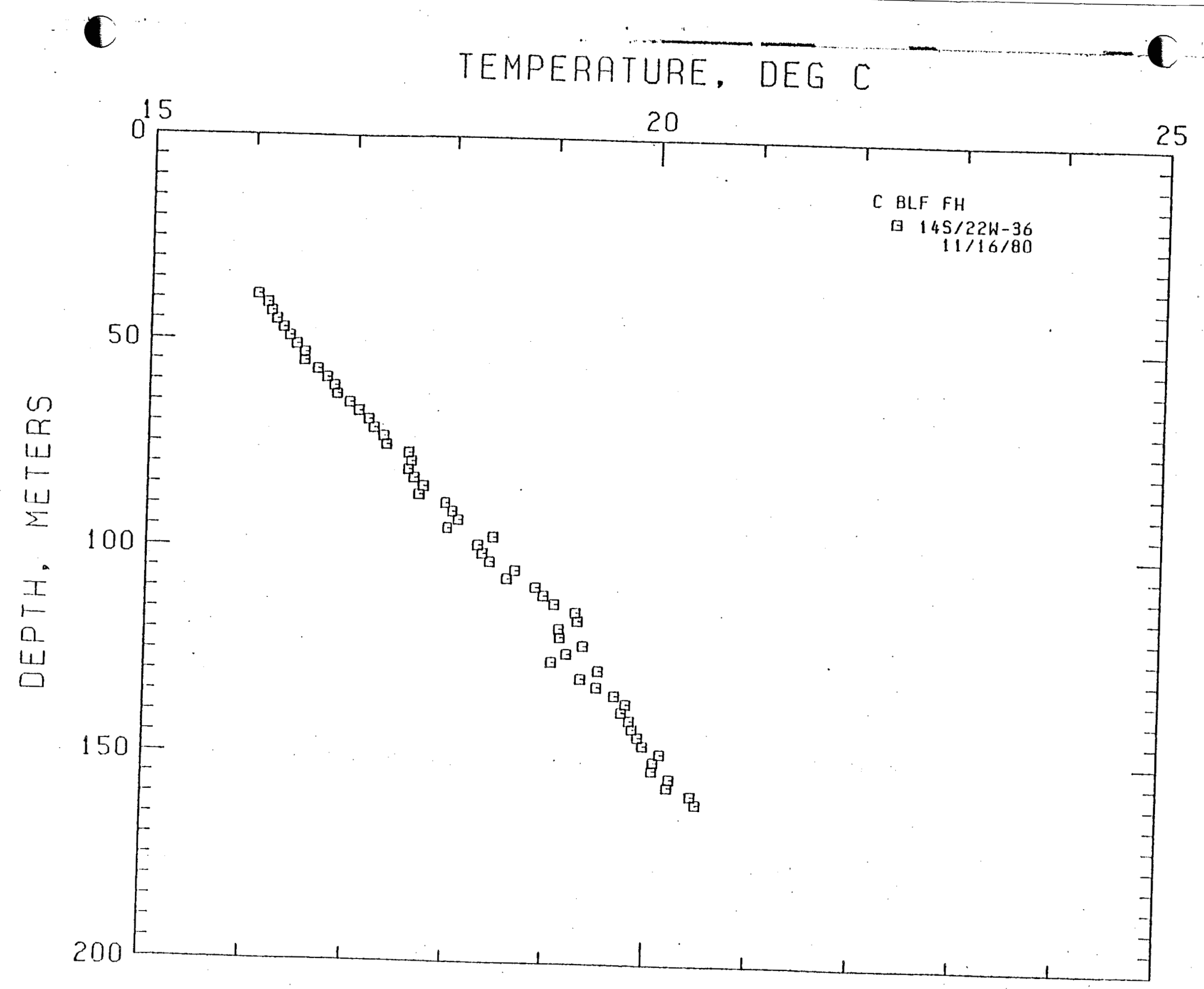




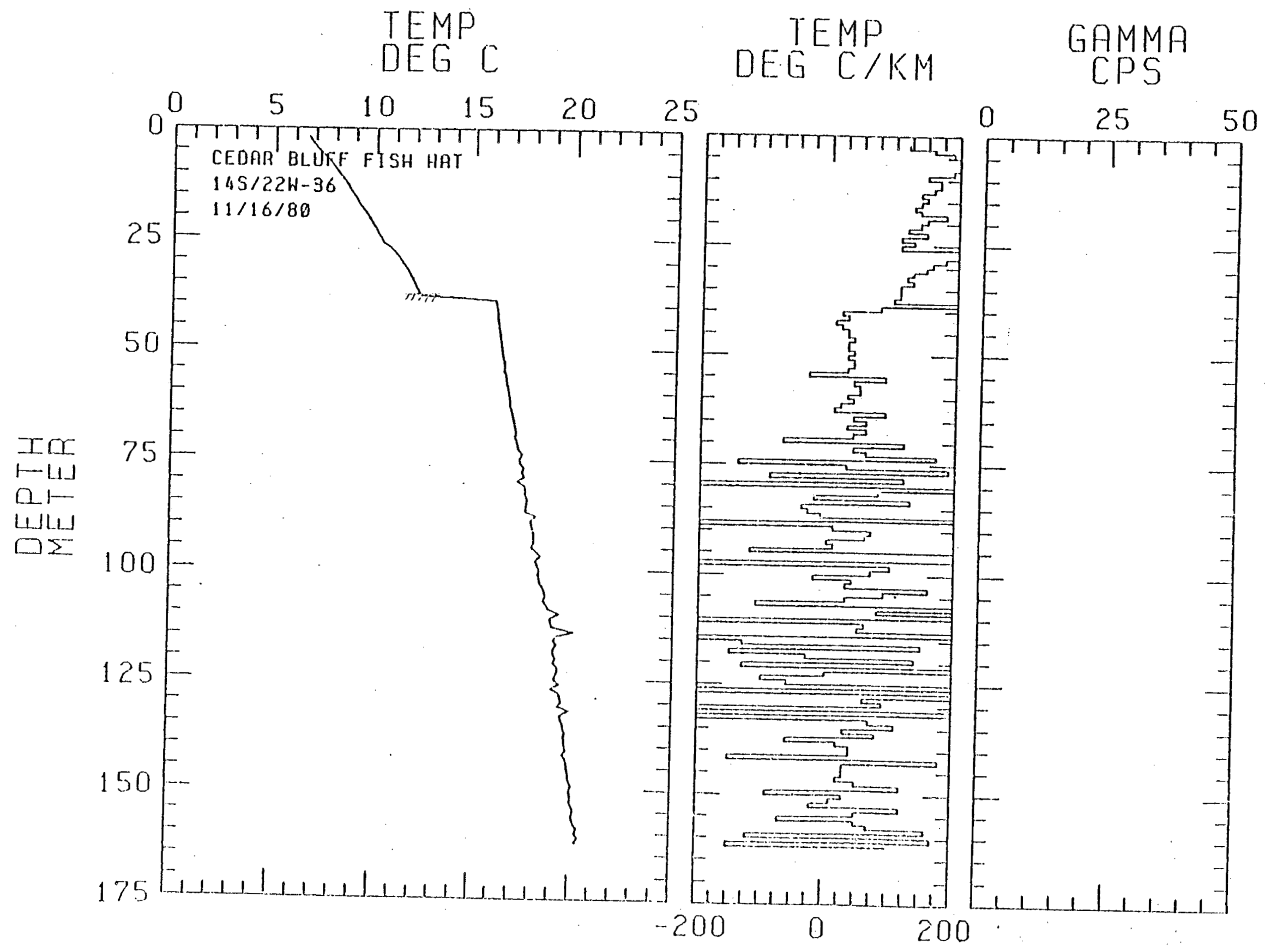




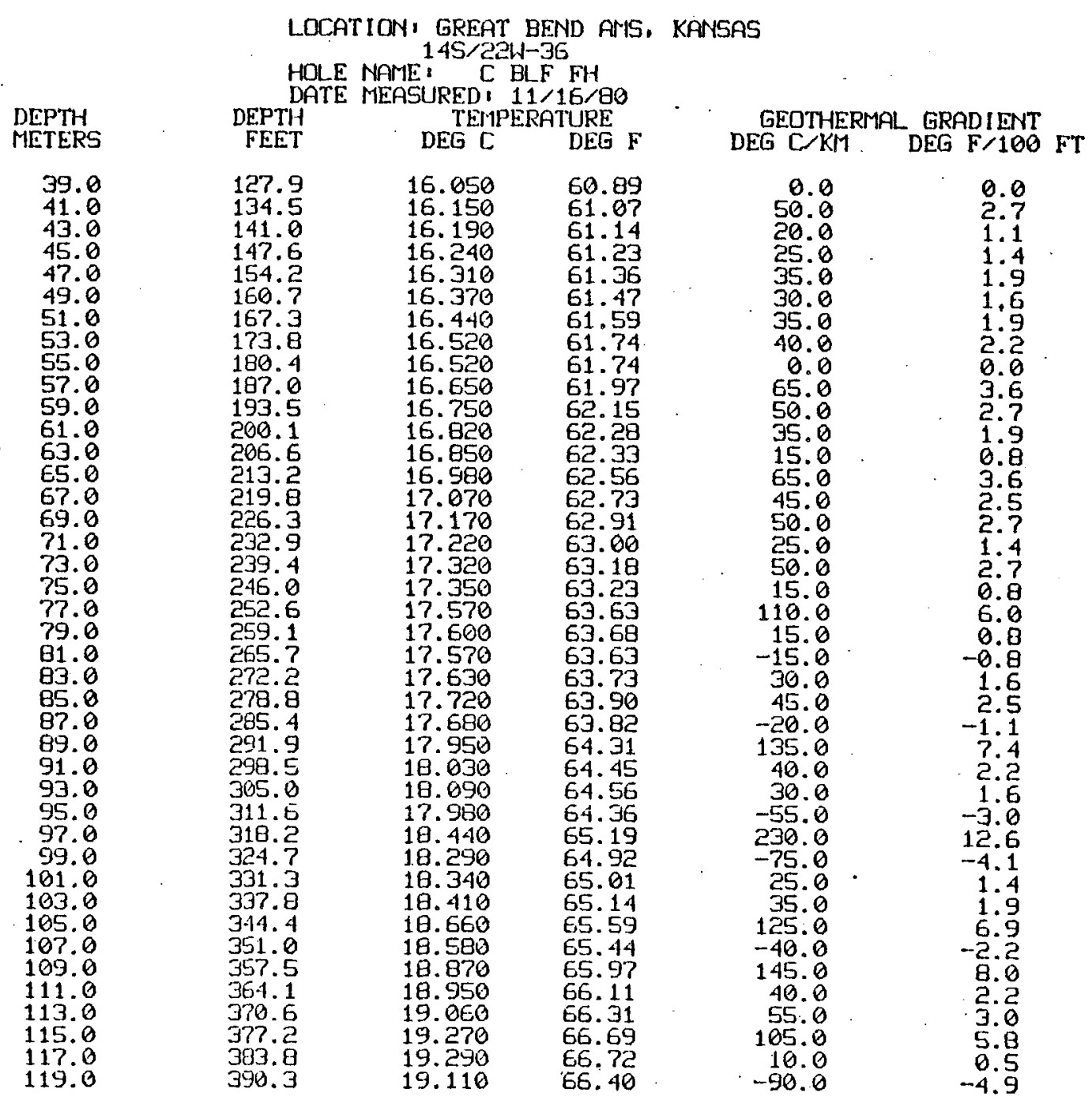




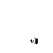

DEPTH
METERS

LOCATION, GREAT BEND AMS, KANSAS

PAGE 2

HOLE NAME 14S/22W-36

HOLE NAME ¿ BLF FH TEMPERATURE

DEG F

GEOTHERMAL GRADIENT

121.0

FEET

66.42

396.9

19.120

410.0

19.190

66.54

127.0

416.6

19.190

19.500

429.1

131.0

436.2

19.370

442.9

449.4

19.490

135.8

139.0

141.0

19.670

19.740

462.5

469.0

143.0

$14 \% .8$

19.740

19.950

19.910

19.960

488 ?

149.0

153.0

495.7

501.9

508.4

515.0

521.5

20.130

20.070

20.230

20.210

20.440

66.27

67.10
66.79

$6 ? .06$

67.41

67.50

67.58

67.73

67.84

67.93

68.13

68.13

157.0

159.0

20.490

68.41

68.38

68.79
68.88

DEG C $/ \mathrm{KM}$

DEG F/100 FT

$$
\begin{array}{r}
5.0 \\
115.0 \\
-80.0 \\
-75.0 \\
230.0 \\
-85.0 \\
90.0 \\
90.0 \\
55.0 \\
-20.0 \\
40.0 \\
15.0 \\
30.0 \\
25.0 \\
85.0 \\
-30.0 \\
-5.0 \\
85.0 \\
-10.0 \\
115.0 \\
25.0
\end{array}
$$$$
\begin{array}{r}
0.3 \\
6.3 \\
-4.4 \\
-4.1 \\
12.6 \\
-4.7 \\
4.4 \\
4.9 \\
3.0 \\
-1.1 \\
2.2 \\
0.8 \\
1.6 \\
1.4 \\
4.7 \\
-1.6 \\
-0.3 \\
4.7 \\
-0.5 \\
6.3 \\
1.4
\end{array}
$$ 
TEMPERATURE, $\overline{D E G ~ C}$

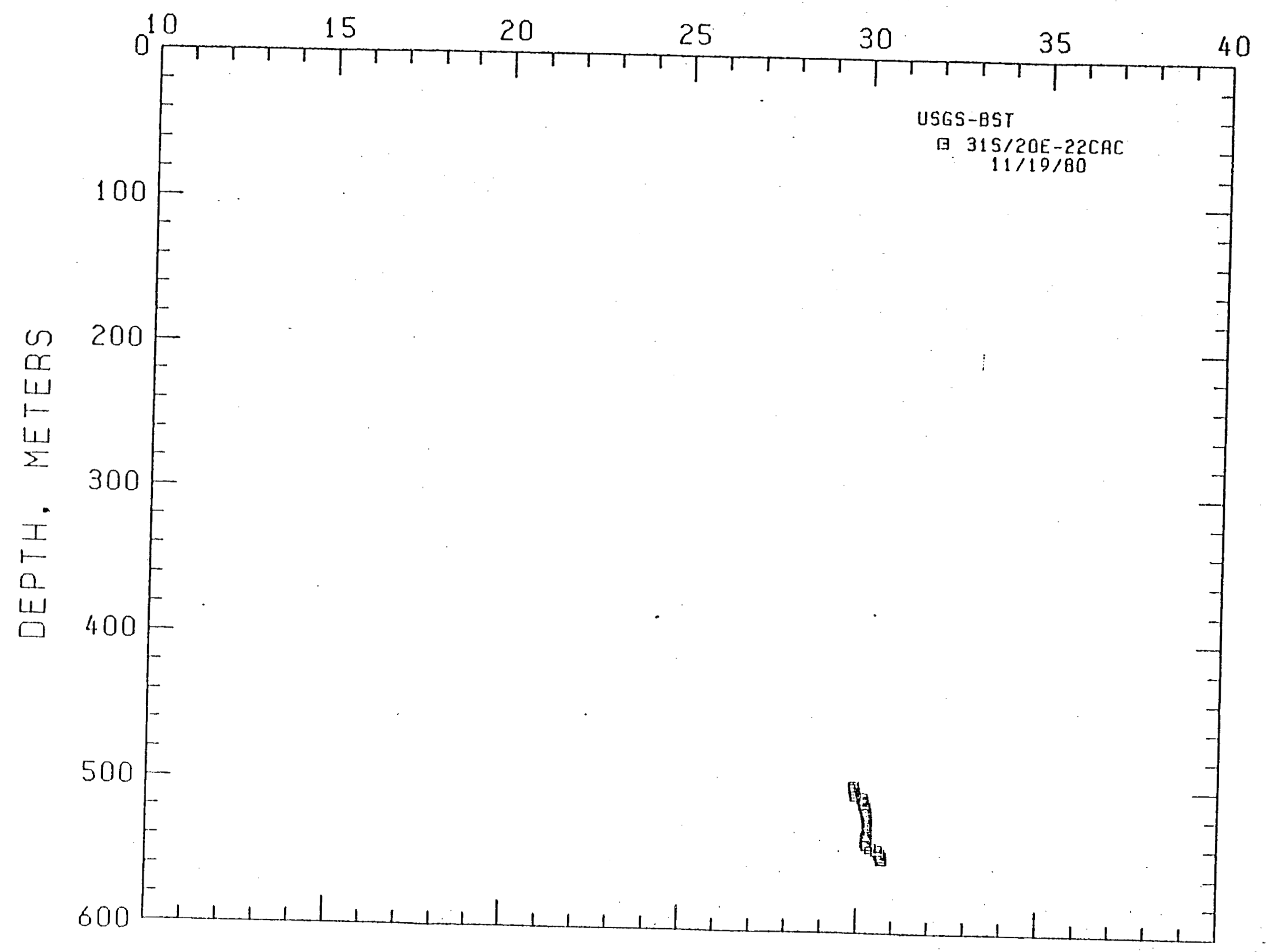




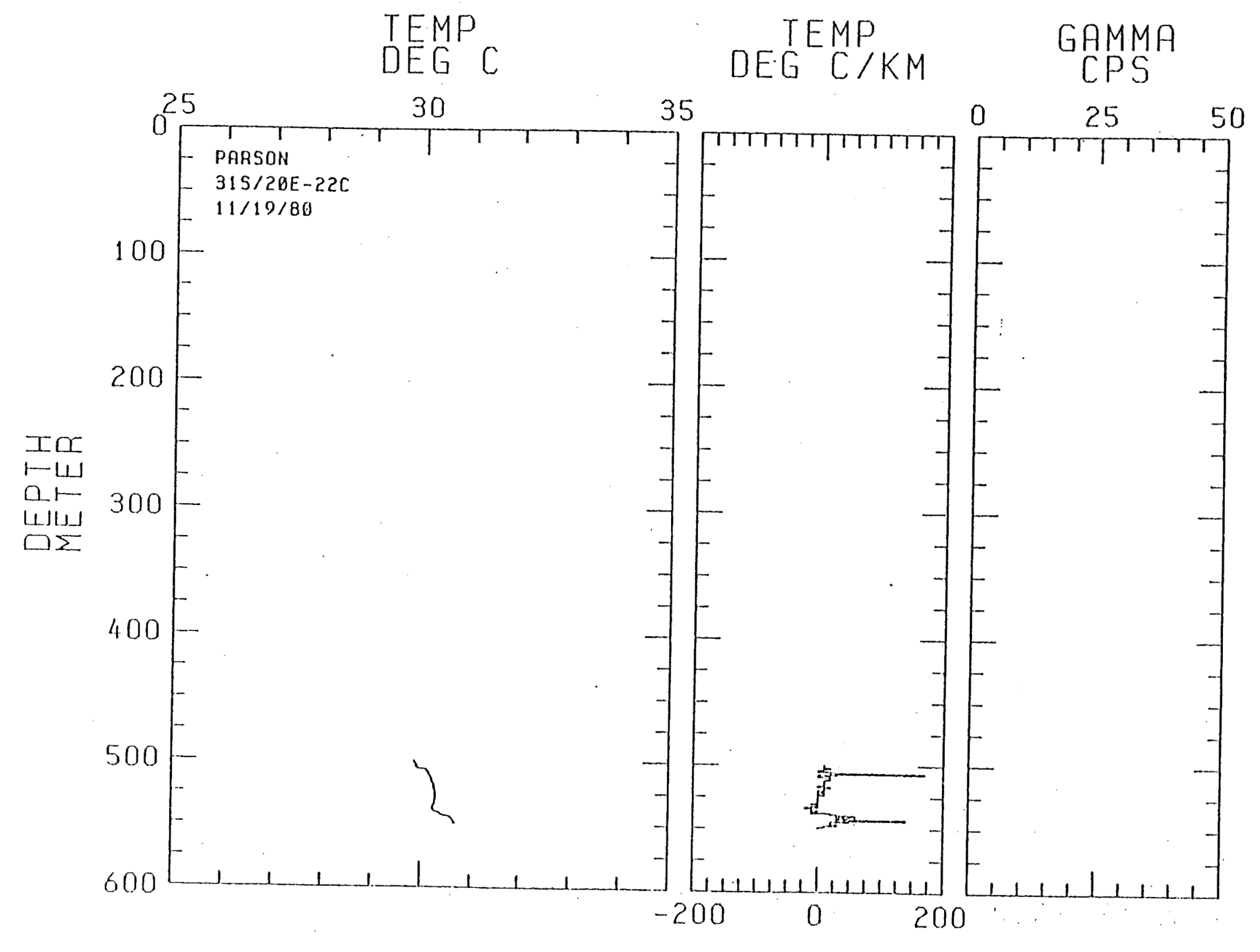




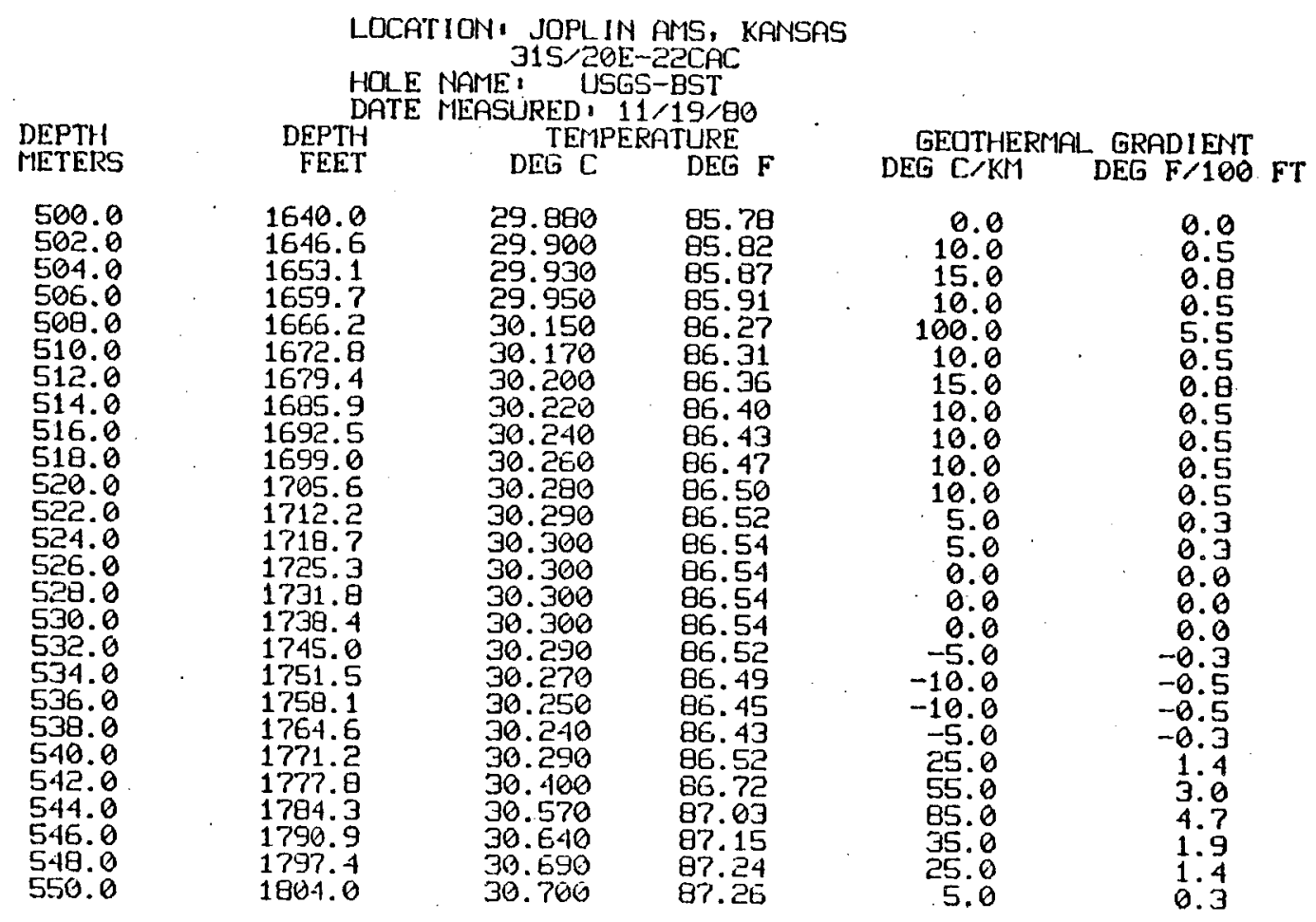




\begin{tabular}{|c|c|c|c|c|c|}
\hline & $\begin{array}{l}\text { Depth } \\
\text { (Eeet) }\end{array}$ & $\begin{array}{c}\text { Eulk } \\
\text { Conauctivity } \\
\mathrm{Nm}^{-1} \mathrm{~K}^{-1} \\
\end{array}$ & & $\begin{array}{l}\text { Depth } \\
\text { feet) }\end{array}$ & $\begin{array}{c}\text { Bulk } \\
\text { Conducivivity } \\
\mathrm{wm}^{-1} \mathrm{k}^{-1} \\
\end{array}$ \\
\hline 300 & -310 & 2.71 & 2100 & -2120 & 2.48 \\
\hline 400 & -410 & 2.76 & & -2160 & 4.82 \\
\hline 500 & -510 & 3.49 & $2200-$ & -2210 & 4.11 \\
\hline 550 & - 560 & 2.47 & $2250-$ & -2250 & 5.02 \\
\hline 570 & -530 & 2.53 & 2300 & -2310 & 4.56 \\
\hline 500 & -610 & 2.69 & $2350-$ & -2360 & 5.46 \\
\hline 700 & -710 & 2.39 & $2400-$ & -2410 & 4.05 \\
\hline 750 & -760 & 2.86 & $2450-$ & -2460 & 5.07 \\
\hline 800 & -810 & 2.33 & $2500-$ & -2510 & 3.93 \\
\hline 340 & -850 & 2.77 & 2550 & -2560 & 5.67 \\
\hline 890 & -900 & 2.69 & 2600 & -2610 & 5.03 \\
\hline 900 & - 910 & 2.81 & 2650 & -2660 & 5.48 \\
\hline 910 & -920 & 3.19 & 2700 & -2710 & 4.36 \\
\hline 920 & -930 & 3.15 & 2750 & -2760 & 5.09 \\
\hline 930 & -940 & 2.68 & 2800 & -2810 & 4.56 \\
\hline 940 & -950 & 2.80 & 2850 & -2860 & 6.10 \\
\hline 960 & -970 & 3.16 & 2900 & -2910 & 4.69 \\
\hline 1000 & -1010 & 3.03 & 2950 & -2960 & 3.94 \\
\hline 1100 & -1110 & 2.63 & & & \\
\hline 1200 & -1210 & 2.25 & & & \\
\hline 1300 & -1310 & $\begin{array}{l}2.41 \\
2.39\end{array}$ & & & \\
\hline $\begin{array}{l}1400 \\
1490\end{array}$ & $\begin{array}{l}-1410 \\
-1500\end{array}$ & $\begin{array}{l}2.39 \\
2.57\end{array}$ & & & \\
\hline $\begin{array}{l}1490 \\
1500\end{array}$ & $\begin{array}{l}-1500 \\
-1510\end{array}$ & $\begin{array}{l}2.57 \\
3.28\end{array}$ & & & \\
\hline 1500 & $\begin{array}{l}-1510 \\
-1530\end{array}$ & $\begin{array}{l}3.28 \\
3.61\end{array}$ & & & $\begin{array}{c}\text { Cere } \\
\text { Conductivity }\end{array}$ \\
\hline 1530 & -1540 & 3.10 & & & $\mathrm{wm}^{-1} \mathrm{k}^{-1}$ \\
\hline 1560 & -1570 & 3.12 & & & \\
\hline 1580 & -1500 & 2.66 & $2958 \cdot 8$ & '8" & 3.22 \\
\hline 1500 & -1610 & 4.76 & $2970 \cdot 5$ & $5^{\prime \prime}-2970^{\prime} 6^{\prime \prime}$ & 3.21 \\
\hline 1620 & -1530 & 3.36 & 297411 & $1.5 "-2974 \cdot 2.5$ & 3.22 \\
\hline 1540 & -1650 & 3.28 & 297713 & $3^{\prime \prime}-2977^{\prime} 4^{\prime \prime}$ & 3.22 \\
\hline $\begin{array}{l}1660 \\
1680\end{array}$ & $\begin{array}{l}-1570 \\
-1690\end{array}$ & 2.94 & & & \\
\hline 1680 & $\begin{array}{l}-1690 \\
-1700\end{array}$ & $\begin{array}{l}2.92 \\
2.62\end{array}$ & & & \\
\hline 1700 & -1710 & 3.14 & & & \\
\hline $1710-$ & -1720 & 3.21 & & & \\
\hline $1720-$ & -1730 & 3.26 & & & \\
\hline $1740-$ & -1750 & 3.23 & & & \\
\hline $1760-$ & -1770 & 3.11 & & & \\
\hline $1780-$ & -1790 & 3.11 & & & \\
\hline $1800-$ & - 1310 & 3.05 & & & \\
\hline $\begin{array}{l}1810- \\
1320-\end{array}$ & $\begin{array}{l}-1320 \\
-1830\end{array}$ & $\begin{array}{l}3.48 \\
3.48\end{array}$ & & & \\
\hline 1900. & $\begin{array}{l}-1830 \\
-1910\end{array}$ & $\begin{array}{l}3.43 \\
2.79\end{array}$ & & & \\
\hline $1990-$ & -2000 & 2.93 & & & \\
\hline $2000-$ & -2010 & 3.04 & & & \\
\hline $20 \leq 0-$ & -2060 & 2.51 & & & \\
\hline $2080-$ & -2090 & 2.45 & & & \\
\hline
\end{tabular}


Smoky HiII, Kansas 13S/2W-32000

$\begin{array}{cc}\begin{array}{c}\text { Depth } \\ \text { Leet) }\end{array} & \begin{array}{c}\text { Bulk } \\ \text { Conductivity } \\ \text { wm-1k-1 }\end{array} \\ 950-960 & 3.39 \\ 905-970 & 3.64 \\ 1700-1710 & 2.70 \\ 1750-1750 & 3.22 \\ 1860-1870 & 2.80 \\ 1940-1950 & 2.82 \\ 2010-2020 & 2.62 \\ 2100-2110 & 2.96 \\ 2150-2160 & 2.98 \\ 2250-2270 & 2.74 \\ 2350-2360 & 2.98 \\ 2440-2450 & 2.32 \\ 2560-2570 & 2.68 \\ 2650-2560 & 3.04 \\ 2700-2710 & 3.86 \\ 2760-2770 & 4.21 \\ 2860-2870 & 2.65 \\ 2950-2960 & 2.54 \\ 3020-3030 & 3.84 \\ 3070-3080 & 3.26 \\ 3130-3140 & 3.28 \\ 3150-3160 & 2.82 \\ 3210-3220 & 2.77 \\ 3250-3260 & 3.12 \\ 3345-3355 & 2.97 \\ 3400-3410 & (2.311 \\ 3446-3456 & 3.72 \\ 3500-3510 & 4.53 \\ 3550-3560 & 4.35 \\ 3600-3610 & 3.90 \\ 3650-3650 & 4.97 \\ & \end{array}$


Watson-I, ãnsas 18s/23E-18cc

$$
\begin{gathered}
\text { Depth } \\
\text { (feet) } \\
350-355 \\
405-410 \\
450-455 \\
505-510 \\
550-555 \\
600-605 \\
650-655 \\
700-705 \\
755-760 \\
770-775 \\
820-825 \\
850-135 \\
895-900 \\
945-950 \\
995-1000 \\
1050-1055 \\
1105-1110 \\
1150-1155 \\
1200-1205 \\
1210-1215 \\
1250-1255 \\
1355-1360 \\
1450-1455 \\
1550-1555 \\
1650-1655 \\
1745-1750 \\
1855-1860
\end{gathered}
$$

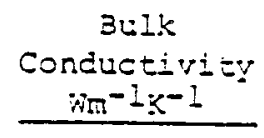

4.11

2.72

2.89

2.92

2.41

2.52

2.98

2.63

2.78

3.02

3.23

2.40

2.90

3.21

4.46

3.12

3.59

3.95

2.28

2.96

3.85

4.32

4.50

4.85

4.38

5.13

5.29 


\section{Bgoendix I}

Natural Remone Uaneziations (No:)

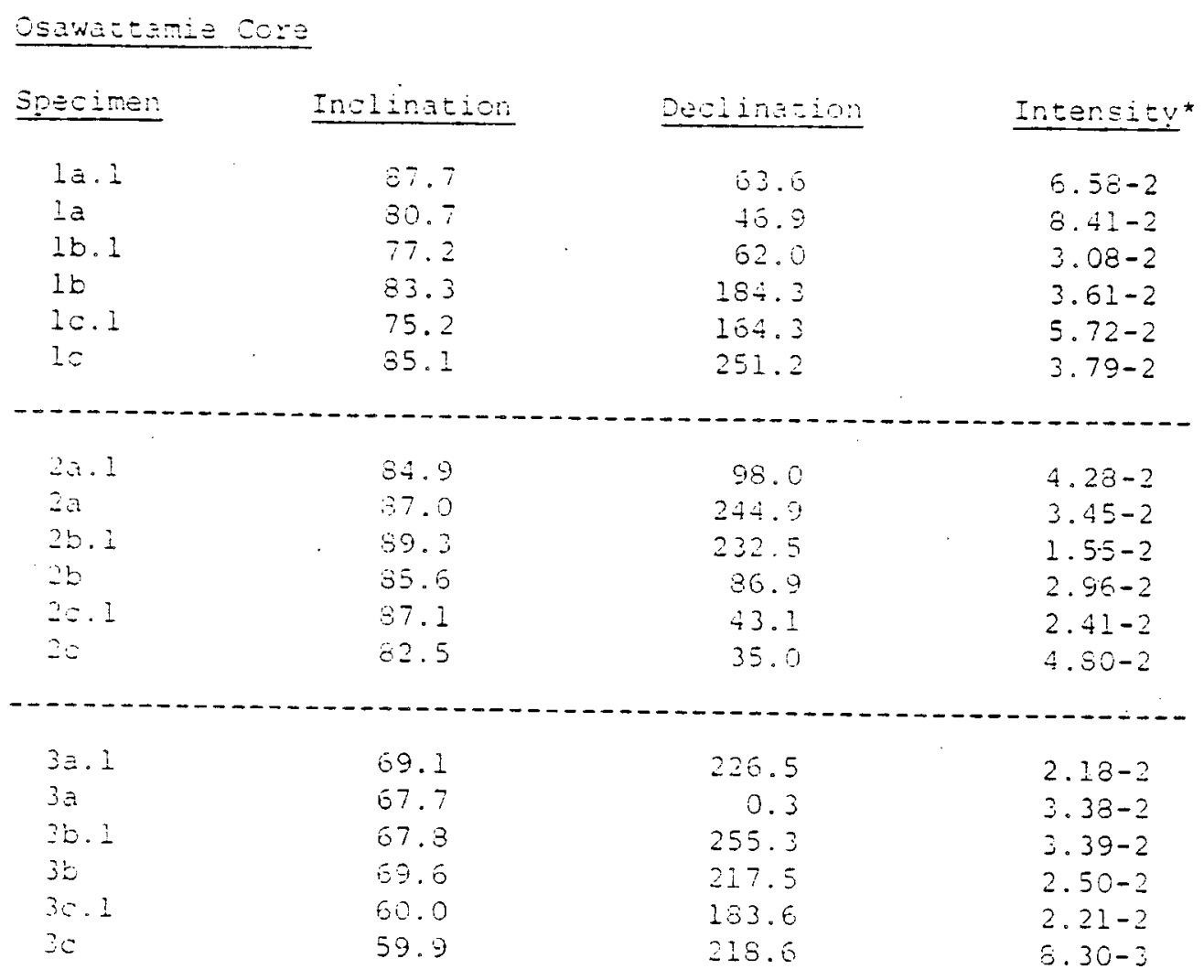

\section{3ig Springs Core}

\begin{tabular}{|c|c|c|c|}
\hline Specimen & Inclination & Declination & Intensity* \\
\hline $\begin{array}{l}1 A \\
1 E \\
1-C-1\end{array}$ & $\begin{array}{l}54.2 \\
51.2 \\
59.4\end{array}$ & $\begin{array}{r}219.1 \\
25.9 \\
35.8\end{array}$ & $\begin{array}{l}1.10-2 \\
1.14-2 \\
1.17-2\end{array}$ \\
\hline $\begin{array}{l}2 \therefore \\
23 \\
2-C-1\end{array}$ & $\begin{array}{l}-77.6 \\
-84.2 \\
-77.7\end{array}$ & $\begin{array}{r}322.3 \\
46.3 \\
321.3\end{array}$ & $\begin{array}{l}3.21-3 \\
2.75-3 \\
1.03-3\end{array}$ \\
\hline $\begin{array}{l}3 \mathrm{~A} \\
3 \mathrm{~B} \\
3-C-1 \\
3-C-2\end{array}$ & $\begin{array}{l}34.3 \\
43.6 \\
39.3 \\
48.3\end{array}$ & $\begin{array}{l}240.9 \\
212.8 \\
260.7 \\
262.9\end{array}$ & $\begin{array}{l}4.73-3 \\
4.58-3 \\
2.36-3 \\
2.66-3\end{array}$ \\
\hline $\begin{array}{l}15 \\
\div S \\
1-C-1\end{array}$ & $\begin{array}{l}66.6 \\
52.5 \\
72.2\end{array}$ & $\begin{array}{l}275.2 \\
270.2 \\
23 \cdot i .2\end{array}$ & $\begin{array}{l}\text { 3.91-3 } \\
1.91-2 \\
3.49-3\end{array}$ \\
\hline
\end{tabular}

*atensties: To chiculat mignetsation, J, civide by Gyogme:n volum $=12.67 \mathrm{gm}^{3}$ 


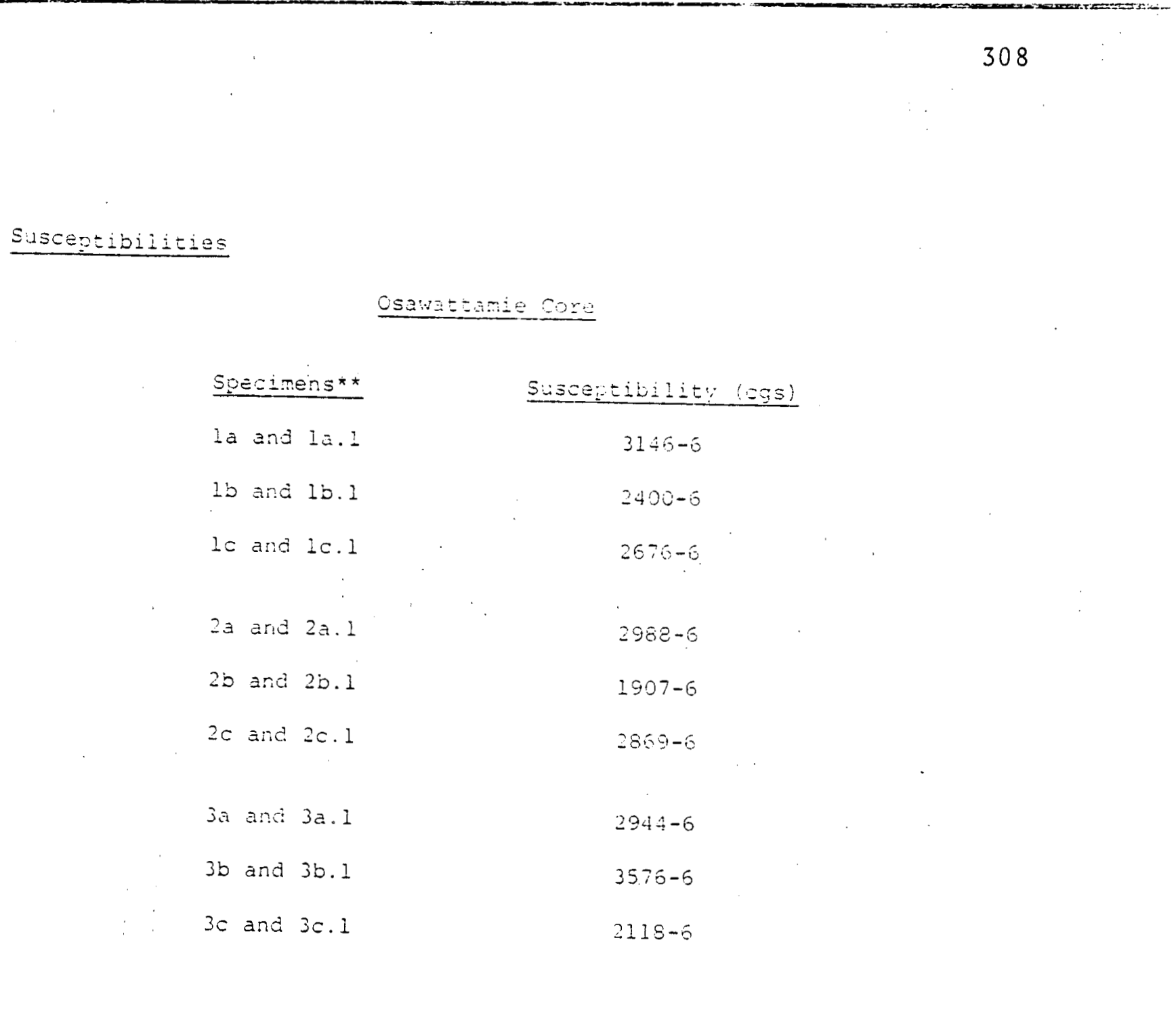

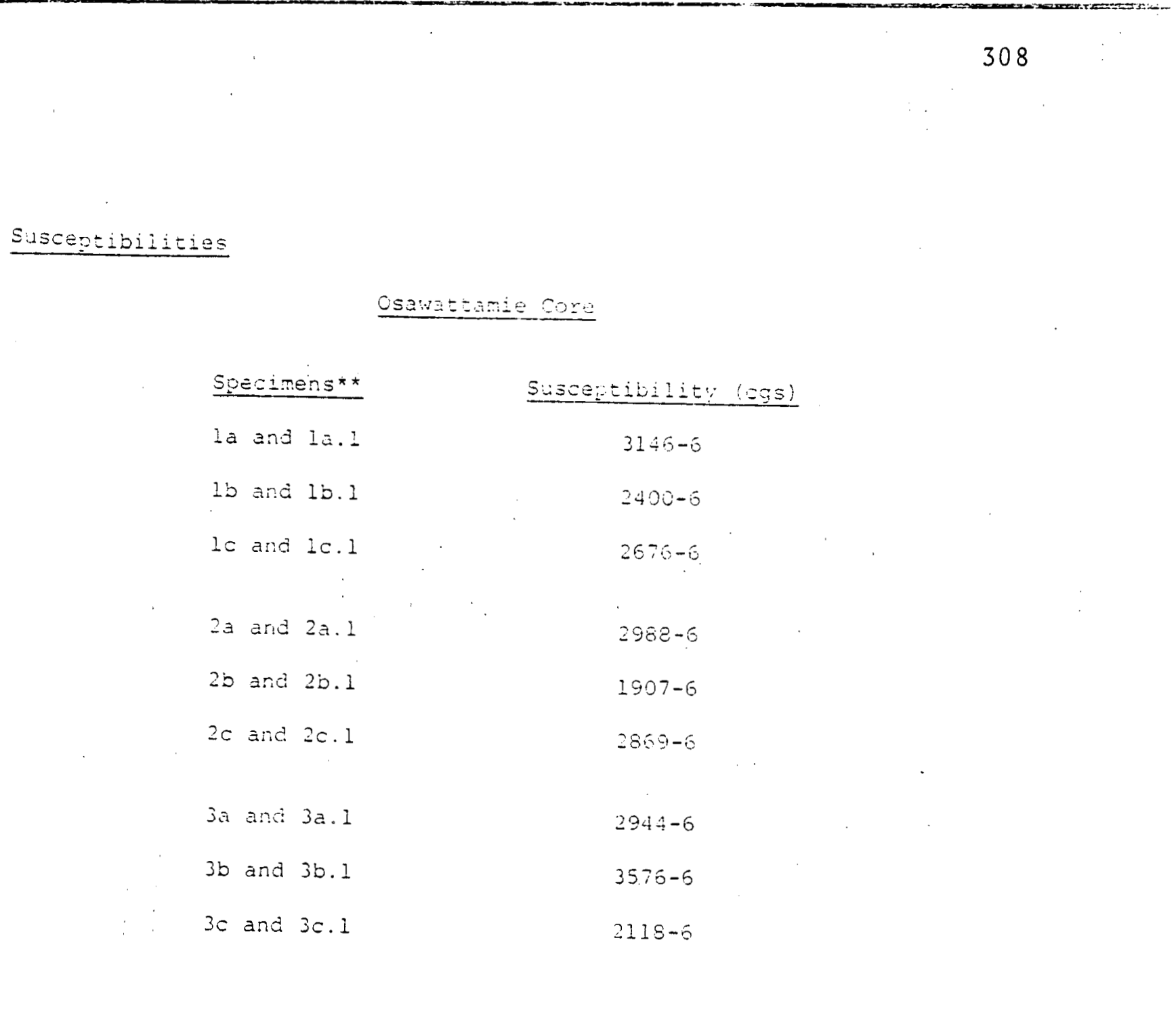

$\begin{array}{lc}\text { Specimens*x } & \text { Suscetibility (ogs) } \\ \text { la and } I a .1 & 3145-6 \\ 1 b \text { and } 1 b .1 & 2400-6 \\ 1 c \text { and } 1 c .1 & 2676-6 \\ 2 a \text { and } 2 a .1 & 2988-6 \\ 2 b \text { and } 2 b .1 & 1907-6 \\ 2 c \text { and } 2 c .1 & 2859-6 \\ 3 a \text { and } 3 a .1 & 2944-6 \\ 3 b \text { and } 3 b .1 & 3575-6 \\ 3 c \text { and } 3 c .1 & 2118-6\end{array}$

* Two paleomagnetic specimens were combined for one susceptibility measurement.

\begin{tabular}{|c|c|c|}
\hline $\begin{array}{c}\text { Big Springs core } \\
\text { Samples } \star \star \star\end{array}$ & \multicolumn{2}{|c|}{ Susceptibility } \\
\hline 1 & $3936-6$ & $\sigma=797-\sigma$ \\
\hline 2 & $2209-6$ & $\sigma=1039-6$ \\
\hline 3 & $.1044-6$ & $\sigma=95-\overline{0}$ \\
\hline 4 & $2622-6$ & $\sigma=680-6$ \\
\hline
\end{tabular}

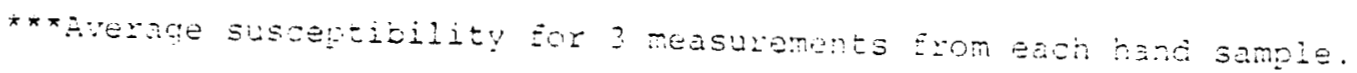


ACTIIAL AND CHEMICAL-gEOTHERMOMETER TEMPERATUGES FOR KANSAS HELL HATERS

\begin{tabular}{|c|c|c|c|c|c|c|c|c|}
\hline \multicolumn{3}{|c|}{ WELL LOCATION } & \multirow{2}{*}{$\begin{array}{l}\text { ACTUAL } \\
\text { TEMP } \\
\text { DEGREES C }\end{array}$} & \multicolumn{5}{|c|}{ GE OIHERMOMEIER IEMPERAIURES - UEGREES C } \\
\hline TI) FNSHIP-RANGE & LINGI IUUE & LATI TUDE & & OUAKT? & CHALCEDONY & $N A-K$ & $N A-K-C A$ & $N A-K-C A$ \\
\hline 13334 acoc & -101.555 & 39.9894 & 16.0 & 101.0 & $E 9.2$ & 197.8 & 14.5 & 30.7 \\
\hline 15394 AOCC & -101.607 & 39.9750 & 17.0 & 84.8 & 51.8 & 191.2 & 69.5 & 25.2 \\
\hline $1539: 34004$ & -101.675 & 39.3189 & 17.0 & 102.8 & 11.2 & 259.4 & G6.5 & 19.6 \\
\hline $1542: 912 A$ & -102.031 & 39.91951 & 17.0 & 95.0 & 62.4 & 295.6 & 19.3 & 27.2 \\
\hline 2338428047 & -101.563 & 39.8482 & 19.5 & $95 \cdot 1$ & 63.9 & 363.1 & 68.7 & 25.5 \\
\hline DTGกWZEACA & -101.150 & 39.8337 & 16.5 & 101.9 & 70.2 & 185.2 & 83.7 & 30.7 \\
\hline$\therefore 740.29 \mathrm{DPA}$ & -101.411 & .39 .9501 & 17.0 & 114.5 & 84.0 & 327.5 & 70.0 & 30.0 \\
\hline 2541428040 & -101.919 & $39.84 \mathrm{BA}$ & 18.5 & 113.7 & 33.1 & 320.1 & 64.0 & $2 H .2$ \\
\hline 233714808 & -101.448 & 39.7975 & 19.0 & 115.3 & 84.8 & 288.5 & 70.1 & 27.4 \\
\hline $2937+1938 C$ & -101.523 & 39.7830 & 19.5 & 116.0 & 85.6 & 288.9 & $\in 9.0$ & $2 n \cdot 5$ \\
\hline$\therefore 5331+2113 \mathrm{CH}$ & -101.598 & 39.1912 & 19.0 & 114.5 & 84.0 & 286.8 & 68.6 & 21.0 \\
\hline $2 S 59: 12 \mathrm{COAC}$ & -101.716 & 39.1758 & 19.0 & 113.0 & 8.2 .3 & 384.5 & 53.7 & 19.0 \\
\hline $3540 \div 38 A C A$ & -101.812 & 39.16667 & 15.0 & 113.1 & 83.1 & 196.7 & 66.9 & 15.8 \\
\hline AS4B:I JSAAC: & -101.772 & 39.7540 & 15.0 & 115.3 & 84.9 & 320.5 & 64.0 & 22.1 \\
\hline 354121604 . & -101.922 & 39.7976 & 17.5 & 113.0 & 82.3 & 423.8 & 56.8 & $1 \mathrm{H.3}$ \\
\hline $354 ? 16000$ & -102.041 & 39.7873 & 18.5 & $11 \subseteq .3$ & 84.9 & 344.2 & $5 H .1$ & 22.3 \\
\hline $453141113 C C$ & -101.449 & 39.7214 & 18.5 & 103.7 & 72.2 & 330.9 & $6 \times .8$ & 22.1 \\
\hline $4 \$ 376179 A C$ & -101.491 & 39.7106 & 18.0 & $10 \mathrm{H} \cdot 1$. & 17.0 & 339.5 & 61.8 & 17.2 \\
\hline $453 \mathrm{H} 250 \mathrm{CA}$ & -101.419 & 39.6725 & 16.5 & 102.8 & 71.2 & 291.6 & 60.0 & 23.5 \\
\hline "S533. 411AC & -101.594 & 34.7396 & 17.5 & 103.7 & $12 . ?$ & 278.8 & $6,4.2$ & 23.7 \\
\hline ASSUW?140C & -101.585 & 39.6925 & 16.5 & 103.7 & 12.2 & 263.2 & 61.7 & 23.2 \\
\hline $4533 \mathrm{H} 20 \mathrm{HC}$ & -101.664 & 39.1323 & 17.0 & 102.7 & 72.2 & 278.7 & 69.6 & $2 i_{3} .5$ \\
\hline 433$)$ d $15 c c A$ & -101.690 & 39.1015 & 11.0 & $10 t .4$ & 75.1 & 210.2 & 66.1 & 25.2 \\
\hline $4539418 C$ AH & -101.144 & 39.7051 & 17.0 & 105.5 & 74.1 & 292.5 & 67.5 & 26.4 \\
\hline $4533 \mathrm{~N} 27 \mathrm{CCA}$ & -101.690 & 39.5725 & 16.5 & $11 \varepsilon_{0} A$ & 86.5 & 276.6 & 60.9 & 22.8 \\
\hline $4 \$ 42: 12 \in B C C$ & -102.011 & 39.0682 & 23.5 & $10 r_{2} .4$ & 15.1 & 318.5 & 50.0 & 8.0 \\
\hline $4: 42 H \leq 4 C A A$ & -102.022 & 39.6619 & 24.5 & 103.7 & $12 . ?$ & 320.1 & 45.2 & 7.9 \\
\hline (5337415043H & -101.459 & 39.6182 & 16.5 & $11 \div .3$ & 84.8 & 250.0 & 59.5 & 18.9 \\
\hline S\$37H18000 & -101.508 & 39.6129 & 17.0 & 113.0 & 82.3 & 259.4 & 56.4 & 16.0 \\
\hline $5833 \cup 17 C H A$ & -131.616 & 39.6183 & 15.0 & 110.6 & 79.1 & 308.9 & 56.0 & 17.9 \\
\hline $5 \$ 3842 E C C A$ & $-101.5 E 0$ & 39.5957 & 16.5 & $11: 00$ & 8.2 .3 & 274.1 & 59.1 & 22.8 \\
\hline $5337411 \mathrm{CHC}$ & -101.674 & 39.6309 & 17.0 & 101.9 & 70.2 & 267.5 & 61.4 & 22.0 \\
\hline$=85941936 \mathrm{C}$ & $-101 \cdot 149$ & 39.6090 & 17.0 & $10 \div .8$ & $18 . d$ & 315.0 & 44.4 & 10.4 \\
\hline$\therefore S 39425 C 0 A$ & -101.649 & 35.5856 & 16.0 & 102.8 & 71.2 & 271.9 & 50.0 & 19.7 \\
\hline S:37L_CCAR & -101.749 & 39.5873 & 15.0 & 111.4 & 80.5 & 231.6 & 61.5 & 20.5 \\
\hline $60+0490 \mathrm{C} n$ & -101.922 & 39.5451 & 15.0 & 114.5 & 84.0 & 250.0 & 63.9 & 24.0 \\
\hline $68+j ! 61418 C 0$ & -101.184 & 39.6198 & 18.0 & 111.4 & 90.5 & 255.2 & 65.4 & 26.0 \\
\hline $5340 \mathrm{U} 2 \mathrm{7BBA}$ & -101.903 & 39.5062 & 17.0 & 108.9 & 17.9 & 224.9 & 58.7 & 18.3 \\
\hline :SAlUIZACC & -101.466 & 39.6342 & $1 t .0$ & 10 月. 1 & 77.0 & 260.5 & 56.4 & 11.0 \\
\hline$=941 \cup 2$ COAA & -101.939 & 39.6035 & 16.5 & 109.1 & 77.0 & 293.9 & 54.9 & 15.5 \\
\hline $5541423 A C 0$ & -101.690 & 39.6052 & 17.0 & $10 ! 31$ & $17 \cdot 13$ & 273.9 & 56.3 & 16.8 \\
\hline $53 A 1$ SInAA & -101.020 & 19.5745 & 16.5 & 101.9 & 30.2 & 293.3 & 56.2 & 17.1 \\
\hline $\cos ?+1400$ & -102.011 & 39.5165 & 16.0 & 95.1 & $\therefore 7.1$ & 308.1 & 13.8 & 12.7 \\
\hline$\therefore 54 ?+301100$ & -101.390 & 39.5165 & 22.0 & 1119.6 & 19.7 & .139 .5 & 49.5 & 7.4 \\
\hline $192 \pi 1926 H A D$ & -100.321 & 34.9424 & 14.5 & 110.6 & 19.7 & 5099.4 & 41.9 & 1.8 \\
\hline 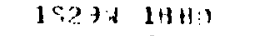 & -100.532 & 40.0804 & 13.5 & $9 A_{0} 1$ & 66.1 & 295.6 & $6,6.1$ & 17.2 \\
\hline $1529 \mathrm{H} \quad 20 \mathrm{CH}$ & -100.557 & 39.9914 & 15.5 & 103.7 & 12.2 & 320.1 & 66.2 & 20.0 \\
\hline 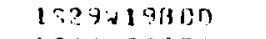 & -100.6 .20 & 39.9533 & 15.5 & 97.1 & 55.0 & 310.4 & 72.5 & $19 . \mathrm{A}$ \\
\hline 1324330000 & -100.520 & 39.9388 & 13.0 & 96.1 & 63.3 & 355.5 & $5 n .1$ & 16.2 \\
\hline $15334: 4000$ & -100.6607 & 39.4172 & 13.5 & 8.6 & 50.3 & 317.5 & 15.1 & 21.8 \\
\hline $252+1113 \mathrm{AHA}$ & -100.709 & 39.4851 & 14.5 & $9 i .1$ & 63.4 & 406.0 & 7306 & 21.8 \\
\hline as? $943: 41+1$ & -100.468 & 39.8427 & 10.5 & 101.3 & 15.13 & 412.8 & 19.1 & 3.2 \\
\hline
\end{tabular}


MELL LOCATION

TOLNSHLP-

\begin{tabular}{|c|c|c|}
\hline$\triangle S 2 A H 32 \mathrm{HCA}$ & -100.495 & 1522 \\
\hline$?: 27 \mathrm{~W} 170 \mathrm{CH}$ & -100.600 & 39.7885 \\
\hline $35234180 C C$ & -100.018 & 39.7966 \\
\hline $55294: 10 \mathrm{CC}$ & -100.618 & 39.7431 \\
\hline $.550 \mathrm{~N} 3 \mathrm{BCO}$ & -100.681 & 39.8229 \\
\hline$\therefore 5300263811$ & -100.665 & 39.7103 \\
\hline "SIEN 1 a HCO & -100.196 & 39.7205 \\
\hline $4 \$ 2 I H 17 D A C$ & -100.371 & 39.7031 \\
\hline $9527 \mathrm{HCOAC}$ & -100.389 & 39.6141 \\
\hline 9827633481 & -100.366 & 39.6685 \\
\hline $4 S 2 A W I 5 O C A$ & -100.429 & 39.6579 \\
\hline 5925W2EDCA & -100.201 & 39.5945 \\
\hline $553043 \mathrm{SuCH}$ & -100.666 & 39.5781 \\
\hline 11538345000 & -100.452 & 39.1190 \\
\hline $11329 \times 21060$ & -100.426 & 39.0755 \\
\hline 11529id 40A1: & -100.535 & 39.1229 \\
\hline $11530027 A B H$ & $-11) 0.6,36$ & 39.0746 \\
\hline $11: 30 \% 310 \mathrm{AH}$ & -100.687 & 39.0534 \\
\hline $11.531 \mathrm{~W}$ 8CHA & -100.790 & 39.1110 \\
\hline 11531 WI $213 \mathrm{HH}$ & -100.719 & 39.1180 \\
\hline $12326+24 \mathrm{CPA}$ & $-100 \cdot 165$ & 38.9926 \\
\hline $1 ? 524.112000$ & $-100 \cdot 372$ & 39.0174 \\
\hline 1?S29:10HAO & -100.532 & 39.0289 \\
\hline 12530W 1AAN & $-100 \cdot 592$ & 39.0436 \\
\hline $13527 \mathrm{~V} 25 \mathrm{AHH}$ & $-100 \cdot 270$ & 38.8994 \\
\hline $13528 \mathrm{~N}$ EHBC & -100.480 & 38.9563 \\
\hline $1752+4 \quad 4 H B 31$ & $-100 \cdot .554$ & 38.9584 \\
\hline $13 S 31 \mathrm{~N}$ SCBA & -100.792 & 38.7532 \\
\hline $19928 W 19 A A B$ & -100.358 & 38.8275 \\
\hline $1 \leqslant \leqslant 264$ ECCR. & -100.244 & 59.7707 \\
\hline $1552 / 42113 A D$ & $-100 \cdot 330$ & 38.7 .397 \\
\hline $15327+13 \mathrm{CCH}$ & -100.503 & 38.7448 \\
\hline ESTAW2BAAIS & -100.007 & 39.51183 \\
\hline 69234331363 & -100.125 & 39.4900 \\
\hline 1522610940 & -99.769 & 33.4628 \\
\hline $752+3 \mathrm{BCHA}$ & -100.029 & 39.4575 \\
\hline 4ระ5แล รดแ & $-100.08 B$ & 39.3485 \\
\hline 5 ระด 6000 & -100.034 & 39.2929 \\
\hline $552.4112 \angle B A C$ & -99.990 & 39.2616 \\
\hline $113.92 \%$ GACC & -100.875 & 39.1271 \\
\hline $1153201513 \mathrm{BH}$ & -100.866 & 39.1037 \\
\hline $11313+10 C$ Ait & -100.973 & 39.1111 \\
\hline DSSSHIEACA & -100.924 & 39.1144 \\
\hline $11534 ! 24000$ & -101.048 & 59.0170 \\
\hline 11814.112200 & -101.149 & 39.1126 \\
\hline $113360 \mathrm{H}, 1 \mathrm{Am}$ & -101.273 & .39 .1324 \\
\hline 11535460111 & -101.351 & 39.1258 \\
\hline 125324 CACH & -100.835 & 39.742 .8 \\
\hline $1259241 \mathrm{SCAA}$ & -100.960 & 39.0104 \\
\hline $1=0,334 \quad 20114$ & -100.751 & 37.0390 \\
\hline 135314 icun & -101.651 & 39.0373 \\
\hline $1 \times 535115000$ & -101.188 & 38.9174 \\
\hline $1 x ; 3+31: B C C A$ & -101.36 .7 & 38.9196 \\
\hline $103352=\operatorname{ccc} 1$ & -101.351 & 38.91050 \\
\hline $1: 5.3 / 415 ! 3 ! n$ & -101.424 & 38.9302 \\
\hline
\end{tabular}

ACIUAL

$$
\text { TENP }
$$

DEGREES C

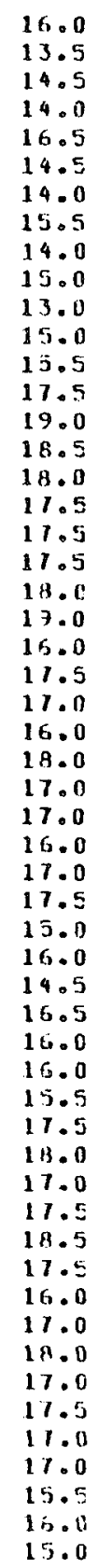

GE OIHFKMOMEJER IEMPERATURES - DEGREES C

QUAKTZ CHALCEDONY NA-K NA-K-CA NA-K-CA LO

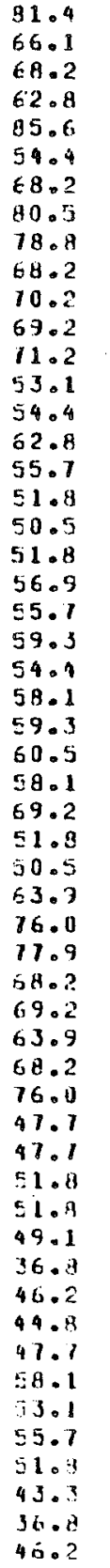

\begin{tabular}{|c|c|}
\hline 423.8 & 56.8 \\
\hline 309.1 & 85.2 \\
\hline 403.5 & 18.2 \\
\hline 364.2 & 66.9 \\
\hline 336.2 & 5.7 .3 \\
\hline 383.4 & 13.9 \\
\hline 424.4 & 51.5 \\
\hline 474.0 & 45.7 \\
\hline 349.4 & 50.8 \\
\hline 418.2 & 54.3 \\
\hline 384.0 & 51.6 \\
\hline 553.0 & 43.9 \\
\hline 491.4 & 45.4 \\
\hline 333.6 & 43.2 \\
\hline $4 B 1.3$ & $2 B .3$ \\
\hline 353.6 & 94.1 \\
\hline 306.7 & 96.0 \\
\hline 355.2 & 39.2 \\
\hline 306.1 & 45.7 \\
\hline 329.6 & 47.5 \\
\hline 270.2 & 38.9 \\
\hline 973.9 & 33.9 \\
\hline 366.9 & 45.8 \\
\hline 326.9 & 44.2 \\
\hline 231.3 & 50.2 \\
\hline 262.9 & 6.3 .2 \\
\hline 286.8 & 15.2 \\
\hline 274.4 & 90.7 \\
\hline $392 \cdot 3$ & $4 ? .5$ \\
\hline 278.8 & 54.6 \\
\hline 321.1 & 51.5 \\
\hline 332.1 & 72.3 \\
\hline 507.6 & 38.5 \\
\hline 501.3 & 39.7 \\
\hline 432.2 & 25.0 \\
\hline 656.3 & 32.6 \\
\hline 416.6 & 55.4 \\
\hline 524.1 & 29.2 \\
\hline 469.2 & 23.5 \\
\hline 529.3 & 30.7 \\
\hline 299.3 & 450.7 \\
\hline 271.3 & 50.0 \\
\hline 314.6 & 47.0 \\
\hline 256.2 & 41.1 \\
\hline 24404 & 46.5 \\
\hline 390.1 & 37.4 \\
\hline 451.5 & 37.0 \\
\hline 314.5 & 42.5 \\
\hline 281.9 & 45.8 \\
\hline 205.5 & 46.5 \\
\hline 282.1 & 50.2 \\
\hline 201.2 & 51.0 \\
\hline 174.0 & 66.0 \\
\hline 164.4 & 62.5 \\
\hline 141.2 & 16.9 \\
\hline
\end{tabular}

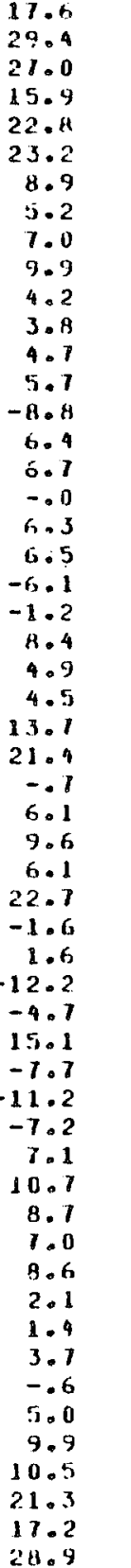


HELL LOCAIION

\begin{tabular}{|c|c|c|}
\hline WNSHIF-RANGE & LONGI TUDE. & LAT \\
\hline$? 5321140 \mathrm{CA}$ & -100.876 & 39.8756 \\
\hline 25324200110 & -100.941 & 39.8028 \\
\hline $2536 \mathrm{~W} 1 \mathrm{HCCH}$ & -101.410 & 39.9753 \\
\hline $2535+36+11 A 1$ & -101.310 & 39.8431 \\
\hline S5314 1BAA & -101.085 & 39.4282 \\
\hline SC.3+H26HAC: & -101.107 & 39.7685 \\
\hline .034123460 & -101.149 & 39.7504 \\
\hline $2355: 124 \mathrm{CH}$ & -101.205 & 39.1776 \\
\hline $\operatorname{ss35:4\operatorname {sec}0}$ & -101.352 & 39.8158 \\
\hline $\operatorname{ssc} \sin 140 \mathrm{col}$ & -101.336 & 39.1923 \\
\hline 39.36w1 JCC. & $-101 \cdot 392$ & 39.7867 \\
\hline 3.s36w22ccn & $-101 \cdot 353$ & 39.7723 \\
\hline 4331 W $160 \cap C$ & -100.745 & 39.6560 \\
\hline $13331113 \mathrm{COMi}$ & -100.981 & 39.7052 \\
\hline $4 S 34429 C A A$ & -101.086 & 39.6906 \\
\hline $43.54233 \mathrm{CBO}$ & $-101 \cdot 150$ & 39.6598 \\
\hline 45362 EHSHIH & -101.411 & 39.7413 \\
\hline AS3SH 9CRD & -101.367 & 39.7143 \\
\hline 4 5.6.ง? $30 \mathrm{CHn}$ & -101.337 & 39.6908 \\
\hline $153642.40 \mathrm{CO}$ & -101.358 & 39.6709 \\
\hline$\because C 3111$ IGAAD & -100.835 & 39.6090 \\
\hline $5532 \pi 36 \mathrm{CDH}$ & $-100 \cdot 866$ & 39.5110 \\
\hline 5.33430040 & -101.064 & 35.5874 \\
\hline $5934426 A C A$ & -101.101 & $39.592 \mathrm{~A}$ \\
\hline $5 \$ 34 \times 2 B A D C$ & -101.136 & 39.5909 \\
\hline$-\$ 27420 \mathrm{Cn}$ & -100.317 & 39.5544 \\
\hline $6927 \because 2 \mathrm{HCC}$ & -100.329 & 39.5035 \\
\hline r\$294100 IIC & -100.542 & 39.5439 \\
\hline (..52)प244 III & -100.505 & 39.5239 \\
\hline E.30N 211CA & -100.642 & $39.56,36$ \\
\hline $63301114 C \mathrm{CD}$ & -100.642 & 39.5255 \\
\hline IS.26HIZBAC & -100.176 & 39.4628 \\
\hline $1526+15 \mathrm{CCH}$ & -100.2 .18 & 39.4395 \\
\hline $7926 \times 130$ UC. & -100.273 & 39.4 .34 .3 \\
\hline $192 /$ KIInan & $-100 \cdot 322$ & 39.4490 \\
\hline TS2BHI9HAAA & -100.494 & 39.9362 \\
\hline $1924: ! 239 A 1$ & -100.415 & 39.4347 \\
\hline IS2 GWSEAUIA & -100.392 & 39.9674 \\
\hline 752918901913 & -100.552 & 39.41104 \\
\hline $7.329 \times 178 \mathrm{HB}$ & -140.589 & 39.4513 \\
\hline $7329 \mathrm{~N}=1 \mathrm{~A} 1111$ & -100.561 & 39.1368 \\
\hline $7521 \mathrm{~W} / \mathrm{decc}$ & -100.552 & 39.4096 \\
\hline 13294301114 & -100.596 & 39.4 .223 \\
\hline $153003 \mathrm{cDABS}$ & -100.705 & 39.1119 \\
\hline $76.30435410 n$ & -100.642 & 39.1059 \\
\hline 13221114044 & -109.184 & .99 .35557 \\
\hline "S? I"1 22110ล & $-100 \cdot 125$ & 33.3455 \\
\hline 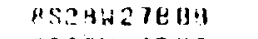 & -100.441 & .39 .3349 \\
\hline 4529i, $101: C$ & -100.506 & 39.31941 \\
\hline MS29'N20ABH & -100.580 & 39.3497 \\
\hline AS29UЕZHAA & -100.582 & $39.3 \times 52$ \\
\hline $\operatorname{sis} 3011 \mathrm{CBC}$ & -1100.0145 & 39.3696 \\
\hline ASSOA $3040 C$ & -1100.710 & 39.3 .333 \\
\hline 'SSEA'd qlice & -190.460 & 39.3205 \\
\hline Бร2НH24HA!I & -100.397 & $39.2 \div 03$ \\
\hline
\end{tabular}

ACTUAL

TEMP

DEGREES C
GEUIHERMOMETER TEMPERATURES - DEGHEES C

GUARTZ CHALCEDONY NA-K NA-K-CA NA-K-CA LU

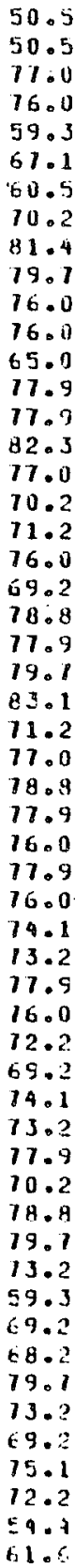

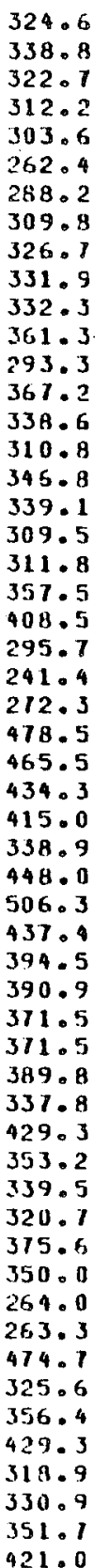

83.0

79.2

12.2

85.9

11.7

73.8

$6 \mathrm{H} .4$

69.4

69.4

70.8

75.0
61.8

61.08
55.0

53.2

61.3

66.8

62.2

60.7

56.0

57.2

54.6

60.2

6.6 .3

64.1

47.6
49.8

53.6

50.0

53.7

51.1

40.1

45.5

48.4

50.4

49.6

43.0

49.9

45.3

49.4

12.6

51.0

55.5

49.7

49.5

42.6

50.6

50.

50.2

40.5

52.7

52.9

51.2
42.4
$32 . A$

29.8

30.9

31.1

20.0

23.2

20.5

23.4

27.6

33.1
24.5

24.5

14.4

13.0

13.5

22.3

25.1

14.9

$1 \mathrm{H} .3$

19.8

A. 3

13.4

24.5

2.8

5.3

12.1

11.0

12. $\mathrm{H}$

11.3

.3

1.2

11.3

10.

10.8
7.2

3.6

10.6

6. 2

11.0

1.9

10.9

20.7

1.6

1.6

1.6

13.2

11.4

1.0

13.1

12.2

2.1 
HELL LOCATION

\begin{tabular}{|c|c|c|c|c|c|c|c|c|}
\hline OOLNSHIF-RANGE & LONGITUDE & LATI TUDE & DEGREES C & QUARIZ. & CHALCELONY & $N A-K$ & $N A-K-C A$ & $N A-K-C A L O$ \\
\hline $7329 \mathrm{N1} / \mathrm{BAH}$ & -100.505 & 39.2772 & $1 \% \cdot 0$ & 75.6 & 36.2 & 327.8 & 45.4 & A. 1 \\
\hline $5527+2344 m$ & -100.589 & 39.2482 & 17.0 & $8 \leq .0$ & 53.1 & 305.5 & 50.8 & 11.6 \\
\hline 5030 A Q AA11 & -100.668 & 39.3962 & 16.5 & 95.0 & 62.8 & 362.0 & 50.5 & 8.9 \\
\hline $593042 C A C C$ & -100.692 & 34.2573 & 16.0 & 82.3 & 99.1 & 946.3 & 10.0 & 1.9 \\
\hline 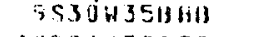 & -106.645 & 39.2337 & 18.0 & 89.9 & 47.7 & 291.1 & 53.4 & 12.4 \\
\hline $11525 \% 32 A C 0$ & -130.245 & 39.1398 & 18.0 & $9 s_{j} \cdot 0$ & $\div 2.8$ & 425.1 & 17.5 & 1.5 \\
\hline $10.528+20 \mathrm{CHC}$ & $-100 \cdot 367$ & 39.1658 & 16.0 & 95.0 & 62.8 & 455.2 & 57.5 & 8.9 \\
\hline 10S2H. 50टH & $-100 \cdot 46.4$ & 39.2079 & 17.5 & 82.3 & 49.1 & 432.7 & 39.6 & .4 \\
\hline $105284270 \mathrm{AA}$ & -100.462 & 39.1534 & 18.0 & 84.8 & 51.8 & 339.9 & 41.1 & 1.2 \\
\hline $1 \mathrm{cssow} 2 \mathrm{zHBO}$ & -100.624 & 39.2029 & 17.5 & $B \in .0$ & 53.1 & 313.1 & 49.0 & 10.2 \\
\hline $105302170 A 13$ & -100.685 & 39.1811 & 17.0 & $\theta \in .0$ & 53.1 & $300 \cdot 6$ & 50.4 & 8.4 \\
\hline $6537 \mathrm{H} 7311 \mathrm{~A}$ & -101.498 & 39.5531 & 13.5 & $9 \subsetneq .1$ & 67.1 & 292.7 & 57.0 & 16.7 \\
\hline COSPW1 6 CDO & -101.956 & 39.5259 & 16.0 & 10504 & 15.1 & 246.2 & 6.0 .9 & 22.5 \\
\hline$\therefore 5314340 \mathrm{cn}$ & -101.433 & 33.4424 & 16.5 & 111.4 & 80.5 & $250 \cdot 8$ & 60.5 & 19.5 \\
\hline ASSBUaCACC & -101.584 & 39.5186 & 14.5 & 105.5 & 14.1 & 308.1 & 53.2 & 15.9 \\
\hline $6: 5394$ 1118:1 & -101.630 & 39.5675 & 16.5 & 108.1 & 77.0 & 241.5 & 61.8 & 23.5 \\
\hline $6533 \times 33400$ & -101.679 & 39.4894 & 16.0 & 110.6 & 79.1 & 294.1 & 48.7 & 12.6 \\
\hline ES4OW1CAAC & -101.765 & 39.5509 & 18.0 & 110.6 & 19.1 & 283.5 & 62.2 & 26.9 \\
\hline$\therefore 941+21 A C C$ & -101.788 & 39.5182 & 16.5 & 112.2 & 81.4 & 258.8 & 60.6 & 24.3 \\
\hline TS41 1ABH & -101.843 & 39.5671 & 16.5 & 118.6 & 19.7 & 242.6 & 53.2 & 13.2 \\
\hline (ES1H190110 & -101.934 & 39.5148 & 17.0 & $10 \epsilon .4$ & 15.1 & 243.2 & 50.5 & 10.5 \\
\hline 83418270417 & -101.878 & 39.5001 & 16.0 & 105.4 & 75.1 & 231.8 & 58.0 & $2 c \cdot 9$ \\
\hline E.542W ACBI3 & $-102 \cdot 540$ & 39.5458 & $1 T .0$ & 108.9 & 77.9 & 279.9 & 47.9 & 15.8 \\
\hline $6.512 \mathrm{W220CC}$ & -101.993 & 39.5113 & 16.5 & 111.1 & 80.5 & 267.3 & 52.0 & 11.5 \\
\hline C542W3CACA & $-102 . n 43$ & 39.5059 & 17.0 & 111.4 & 80.5 & 276.6 & 51.0 & 15.4 \\
\hline $1531 \mathrm{H} 12 \mathrm{Col}$ & -101.408 & 39.4553 & 17.0 & 109.9 & 77.9 & 222.3 & 56.4 & 16.6 \\
\hline 2S.51W17RBA & -101.175 & 39.4479 & 20.0 & 110.6 & 19.7 & 229.1 & 56.5 & 16.5 \\
\hline $75374310 \mathrm{CC}$ & -101.492 & 39.3753 & 16.9 & $10 \subseteq .8$ & 78.8 & 225.3 & 52.6 & 15.0 \\
\hline $1538: 1251304$ & -101.519 & 39.4206 & 16.5 & 11608 & 86.5 & 218.4 & 54.1 & 16.0 \\
\hline $7337498 \mathrm{BHit}$ & -101.686 & 39.4658 & 16.0 & 109.8 & 78.9 & 315.0 & $4 n .6$ & 3.5 \\
\hline $233942913 \mathrm{An}$ & -101.623 & 39.4369 & 16.0 & 112.2 & 81.1 & 230.6 & 53.7 & 14.5 \\
\hline $7539 \mathrm{w} 3 \mathrm{ccc}$ & -101.724 & 39.4113 & 15.0 & $10 n .1$ & 77.0 & 226.0 & 51.4 & 14.6 \\
\hline 15411W GADH & -101.820 & 39.4764 & 16.0 & 113.0 & 82.3 & $24 \mathrm{~A} .9$ & 98.7 & 13.5 \\
\hline $794: 1423110 \mathrm{C}$ & -101.756 & 39.4312 & 15.5 & 110.6 & 79.7 & 288.5 & 42.6 & 8.1 \\
\hline I990k2981:A & -101.414 & 39.4220 & 14.5 & 101.3 & 16.0 & $331 \cdot 5$ & 35.5 & 2.7 \\
\hline 15421458311 & -101.925 & 39.4784 & 16.0 & 112.1 & $2 x^{2}-1$ & 217.1 & 59.1 & 23.0 \\
\hline 3942H2 OBNA & $-101.98 B$ & $39.46,57$ & 16.5 & 110.6 & 79.1 & 238.9 & 50.6 & 15.3 \\
\hline |SA1H2BDPII & -101.900 & 39.1149 & 15.5 & 105.8 & 18.8 & 251.1 & $4 \mathrm{B.3}$ & 13.6 \\
\hline $1542 N 70 A A$ & $-102 . n 43$ & 39.4587 & 16.0 & 101.3 & 16.0 & 210.2 & 57.0 & 17.5 \\
\hline ISA2U27AAII & -101.989 & 39.4223 & 16.5 & 10.3 .1 & 12.2 & 243.0 & 45.03 & 10.1 \\
\hline OS33:124AAH & -101.506 & 39.3499 & 16.0 & 108.1 & 77.0 & 226.3 & 46.2 & 9.1 \\
\hline YS39+2BACC & -101.566 & 39.3299 & 17.0 & 100.0 & 68.2 & 234.1 & 45.0 & R. 1 \\
\hline 95394 ZHIIA & -101.647 & 39.3935 & 15.0 & 107.3 & 76.0 & 214.1 & 49.8 & 10.4 \\
\hline AS3SW1:AAIS & -101.617 & 39.3643 & 17.0 & 109.8 & 78.8 & 205.7 & 19.8 & 11.5 \\
\hline AS33:170CO & -101.694 & 34.3515 & 17.0 & 104.9 & 11.9 & $208 \cdot 5$ & 45.4 & $11 \cdot 9$ \\
\hline OSSOU2IAAH & -101.654 & 39.3 .352 & Lis.o & 100.1 & 71.0 & 215.7 & 46.7 & 11.9 \\
\hline $83100-5013$ & -101.807 & $3 \% .3058$ & 17.0 & 102.8 & 11.2 & 196.3 & 51.5 & 15.9 \\
\hline $5540+1$ aCCU & -101.752 & 37.3532 & 15.11 & 97.1 & 51.1 & 205.1 & 18.7 & 14.9 \\
\hline 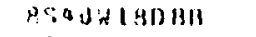 & -101.1126 & $39.3 \sqcup 68$ & 16.0 & $104 . \therefore$ & 13.2 & 187.5 & 54.5 & 19.1 \\
\hline$P 540435 C \mathrm{Cl}$ & -101.162 & 39.3077 & 17.0 & 75.3 & 11.7 & 215.3 & 42.6 & 0.7 \\
\hline HSALW1TCHA & -101.926 & 39.3569 & 16.11 & 100.0 & ن́.? & 321.1 & 34.5 & .7 \\
\hline $65921620 \mathrm{AH}$ & -101.970 & 39.5960 & 16.0 & 100.0 & $6 A .2$ & 245.1 & 3.3 .1 & 11.8 \\
\hline $8.92 \times 19 n 1 m 1$ & -102.051 & 39.9478 & 17.0 & 91.0 & 62.6 & 252.4 & 42.4 & 8.9 \\
\hline $95426340 \mathrm{Cb}$ & -101.994 & 39.31198 & 17.0 & Bii.9 & +7.7 & 216.7 & 50.2 & 10.1 \\
\hline $5039411 B H A$ & -101.104 & 39.2711 & 16.0 & 75.3 & 91.1 & 209.4 & 42.6 & $7 \cdot H$ \\
\hline
\end{tabular}


WELL LOCATION

ACIUAL

DEGREES

17.0
16.0

17.0

$1 \mathrm{r} .0$

16.0

16.0

16.0

17.0

15.0

17.0
15.0

15.0
12.0

19.0
15.0

15.0

15.0

15.0

16.0

17.5
15.0
16.0

16.0

15.5

16.

18.

15.0

16.0
16.0

16.0
17.0

17.0
16.5

17.5

17.0

15.5

15.5
14.5
15.5

14.5
15.5
16.5
18.0

18.0

16.0

15.5

15.0

16.0

15.0

17.0

$16.1)$

15.5

15.5

16.0

15.0

15.5

16.0

16.0

16.0

15.0

1500

15.0

11.0
GE OTHERMOMETER TEMPERATURES - DEGREES C

QUARTZ CHALCEDONY NA-K NA-K-CA NA-K-CALO

$69.0 \quad 35.1$

$\begin{array}{ll}65.0 & 35.1 \\ 73.7 & 36.8 \\ 73.8 & 40.1 \\ 89.6 & 56.5 \\ 10.1 & 36.8 \\ 61.3 & 33.5 \\ 61.8 & 27.5 \\ 72.3 & 20.5 \\ 91.8 & 59.3 \\ 65.5 & 31.9\end{array}$

226.9

39.4

8.8

$194.4 \quad 93.8$

$200.2 \quad 16.3 \quad 17.1$

$204.1 \quad 11.9$

$221.9 \quad 31.7 \quad 4.5$

$203.5 \quad 37.7 \quad 9.2$

$\begin{array}{lll}262.9 & 52.1 & 16.1 \\ 194.9 & 0.9 & 14.0\end{array}$

$259.133 .0 \quad 3.0$

$\begin{array}{lll}153.1 & 43.2 & 3.0 \\ 920.2 & 36.3 & 3.2\end{array}$

$218.9 \quad 47.1 \quad 11.2$

$\begin{array}{lll}271.8 & 34.4 & .6\end{array}$

$\begin{array}{lll}419.5 & 51.1 & 10.7\end{array}$

$353.6 \quad 49.3 \quad 9.2$

$234.6 \quad 61.7$

$389.6 \quad 52.2$

$442.4 \quad 45.1 \quad 5.0$

$62.1 \quad 20.3$

290.62 .4

$\begin{array}{lll}281.5 & 56.4 & 13.5\end{array}$

$308.5 \quad 60.9 \quad 21.0$

$247.3 \quad 64.1 \quad 21.9$

$396.6 \quad 54.7 \quad 14.6$

$254.3 \quad 64.2 \quad 24.9$

$247.1 \quad 51.5 \quad 18.5$

$344.3 \quad 57.5 \quad 18.1$

$303.0 \quad 55.8$

$344.1 \quad 51.9$

$323.6 \quad 52.7$

310.255

302.53

$334.8 \quad 49.1 \quad 10.3$

$313.1 \quad 47.4$

$267.2 \quad 59.6 \quad 23.0$

$310.4 \quad 51.3 \quad 16.8$

$\begin{array}{rrr}265.9 & 50.2 & 12.6 \\ 296.1 & 95.5 & 5.2\end{array}$

$\begin{array}{lll}359.6 & 50.2 & 10.0\end{array}$

$\begin{array}{lll}49.5 & 49.1 & 11.4\end{array}$

$305.0 \quad 50.6$

$320.7 \quad 49.0$

264.8 5a.6

$301.3 \quad 51.2$

$329.5 \quad 5 \times 05$

$316.5 \quad 53.5$

1.. 6

16.4
10.3 
WELL LOCATION

\begin{tabular}{|c|c|c|}
\hline NSHIP - A ANGE & LONGI IUDE & LATITUDE \\
\hline BSSGUGÉnAa & $-101 \cdot 168$ & 39.31 .37 \\
\hline 8.536h 40CC & -101.338 & 39.3410 \\
\hline $853,12 \mathrm{COHH}$ & $-101 \cdot .362$ & 39.3450 \\
\hline $5531: 11 C B+18$ & -100.715 & 39.2918 \\
\hline 9.35419HCA & -100.829 & 39.2593 \\
\hline 9331. $36 B H A$ & -100.738 & 39.2337 \\
\hline$=532 \mathrm{~W} 91 \% \mathrm{DA}$ & -100.1999 & 39.2884 \\
\hline $953242 \mathrm{InCD}$ & $-100 . P B S$ & 39.2430 \\
\hline $5 S 33$ id $6 A A A$ & -10.1 .038 & 39.3067 \\
\hline 95334 gecc & -101.017 & 39.2795 \\
\hline osssid1 SACC & -100.990 & 39.2722 \\
\hline $553343 C C A A$ & -101.048 & 39.2416 \\
\hline 4533435 AAD & -100.964 & 39.232 .2 \\
\hline $553442 B 0 A$ & -101.085 & 39.30 .30 \\
\hline SS34HI JBBA & -102.145 & 39.2775 \\
\hline $9555.320 \mathrm{AA}$ & -101.243 & 39.2265 \\
\hline $10331 \mathrm{H} 60 \mathrm{CA}$ & -100.1320 & 39.2086 \\
\hline $10.931 * 1311$ AI) & -100.731 & 39.1884 \\
\hline 1CS.T1!25AAH & -100.799 & 39.1614 \\
\hline $10532 \mathrm{~A}$ ECAF & -100.939 & 39.2122 \\
\hline 1US32:1110AA & -100.862 & 39.2050 \\
\hline $10532: 1290 \mathrm{Cll}$ & -100.916 & 39.1505 \\
\hline $10532 \mathrm{H} 3 \mathrm{CAAII}$ & -100.935 & 39.1514 \\
\hline $10533 \div 10 D A A$ & -100.983 & 39.1979 \\
\hline $10533 H 17 A A A$ & -101.020 & 39.1908 \\
\hline $14333419 \mathrm{CBO}$ & -101.053 & 39.1674 \\
\hline IOS34W IAI:A & -101.062 & 39.2199 \\
\hline 1853412400 & -101.071 & 39.1999 \\
\hline 10533.5 SAAR & -101.227 & 39.2049 \\
\hline 10S35. 7ACC & -101.381 & 39.2000 \\
\hline I153HA ЕНBC. & -101.569 & 39.1302 \\
\hline 11S3JW2ECCO & $-101 \cdot 6.23$ & 39.0615 \\
\hline 115114553130 & -101.846 & 39.0575 \\
\hline $11 \div 424.5 \mathrm{CH}$ & -102.014 & 39.1246 \\
\hline $11542 \mathrm{HCCC}$ & -102.001 & 39.1046 \\
\hline $1(512 J 1$ (OAAI) & -101.960 & 39.1155 \\
\hline $1953 \% 1420 C 0$ & -101.685 & 38.9023 \\
\hline 12502410483 & -191.149 & 38.3436 \\
\hline 1.541212000 & -101.817 & 38.9307 \\
\hline $14533436140 \mathrm{CH}$ & -101.6 .13 & 38.7961 \\
\hline $10 S 40$ is $23 A C E$ & -101.733 & 38.0225 \\
\hline $1.541 \mathrm{N3HHA}$ & -101.853 & 38.11270 \\
\hline $1454242 A 4 H$ & -101.349 & 38.9699 \\
\hline $1 \cos 3+21100$ & $-101.6,01$ & 38.6930 \\
\hline 1f53-3:11 \&BAC & $-10) 1.640$ & $3 A .6670$ \\
\hline $16: 33: 220 \mathrm{CH}$ & -101.614 & $38.643 n$ \\
\hline 16351250811 & $-101.5 \mathrm{AG}$ & $5 P .6332$ \\
\hline$I F S 402$ EHAA & -1.01 .785 & 38.6769 \\
\hline 1. S\$OU1SACr. & -101.726 & $38 \cdot 155,30$ \\
\hline $16540.25593 A$ & -101.114 & 34.6250 \\
\hline $\operatorname{siseds~scce~}$ & -141.384 & 38.6931 \\
\hline 1:S1 1 2 CHAl! & -101.877 & 38.6506 \\
\hline $16: 42 d 2316 c 18$ & -131.056 & $: 8.6+10$ \\
\hline 19354800 & -101.677 & $.5 B .5920$ \\
\hline $17559: 1901111$ & $-1101.6,64$ & 38.5599 \\
\hline
\end{tabular}

ACTUAL DE GREES C

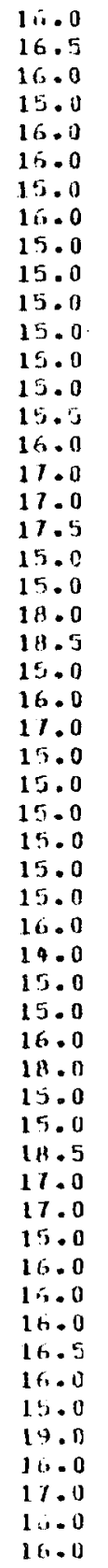

GE OIHERMOMETER TEMPERATURFS - DEGREE C

GUARTZ CHALCEDONY NA-K NA-K-CA NA-K-CA LO

$\begin{array}{rl}91.1 & 65.0 \\ 103.5 & 74.1 \\ 103.5 & 14.1 \\ 83.6 & 50.5 \\ 65.0 & 35.1 \\ 73.8 & 10.1 \\ 73.7 & 36.8\end{array}$

$\begin{array}{lll}25 A .1 & 57.4 & 16.8 \\ 270.7 & 47.1 & 9.3\end{array}$

$252.5 \quad 42.5 \quad 9.3$

3 B1. B $\quad 45.1 \quad 4.0$

$302.3 \quad 55.3 \quad 15.1$

$311.1 \quad 50.9$

$343.1 \quad 96.6 \quad 11.8$

$276.5 \quad 51.3$

$273.8 \quad 14.6$

$273.0 \quad 50.010 .3$

$\begin{array}{lll}260.4 & 49.6 & 10.1 \\ 216.6 & 50.8 & 15.2\end{array}$

$343.543 .1 \quad 5.8$

$\begin{array}{rrr}350.2 & 49.6 & 10.6 \\ 313.1 & 47.4 & 6.3\end{array}$

$265.9 \quad 52.9 \quad 10.8$

$298.7 \quad 5 \times .8 \quad 13.9$

$300.9 \quad 49.6 \quad 10.6$

$279.3 \quad 12.1$

$291.2 \quad 53.4$

$343.1 \quad 41.1 \quad 1.2$

$324.6 \quad 43.9 \quad 3.3$

$255.0 \quad 52.7 \quad 12.0$

$337.8 \quad 39.3 \quad 0.3$

$432.1 \quad 31.6 \quad-5.9$

$\begin{array}{lll}255.5 & 49.4 & 9.5 \\ 343.3 & 41.8 & 6.8\end{array}$

$\begin{array}{lll}343.3 & 41.3 & 9.2\end{array}$

$350.4 \quad 15.2$

$197.9 \quad 64.7 \quad 15.7$

$228.7 \quad 64.9 \quad 19.3$

$199.8 \quad 57.9 \quad 14.6$

$29.8 \quad 39.9 \quad 5.0$

$240.4 \quad 42.5$

$211.5 \quad 64.2$

217.5

$2 B 3.1 \quad 52.0 \quad 10.3$

$225.5 \quad 41.3 \quad 8.4$

$\begin{array}{lll}242.0 & 37.8 & 5.3 \\ 233.9 & 39.4 & 6.3\end{array}$

$281.7 \quad 34.4 \quad 3.4$

$256.2 \quad 52.6$

$250.0 \quad 48.2$

$234.9 \quad 46.4 \quad 3.3$

$299.9 \quad 47.9$

$240.3 \quad 49.9$

$24.5 \quad 46.5$

24.65

$231.1 \quad 41.0 \quad 4.0$

$235.8 \quad 42.2 \quad 6.0$

$\begin{array}{lll}255.5 & 48.5 & 9.6 \\ 276.6 & 42.2 & 5.9\end{array}$ 
WELL LOCATION

\begin{tabular}{|c|c|c|}
\hline GEE & $\operatorname{LON} 5$ & LATITUD \\
\hline $11534422 \mathrm{AH11}$ & -101.614 & 38.5675 \\
\hline $17540 \mathrm{U} 411 \mathrm{CH}$ & -101.744 & 38.5994 \\
\hline $17.54 \mathrm{~J} 115 \mathrm{Cr}$ & -101.735 & 33.5704 \\
\hline $12510 d 31613 A$ & $-1 \cup 1.18 \mathrm{~A}$ & 38.5375 \\
\hline $1 / 542.427 \mathrm{CH}$ & -101.356 & 38.5134 \\
\hline 18530t 711400 & -101.676 & 38.5072 \\
\hline $19539.230 \mathrm{Cl}$ & -101.604 & 38.4695 \\
\hline $19540: 14 C 130$ & -101.751 & 34.5142 \\
\hline $16 \$ 2342 E C(D)$ & -100.501 & 38.6269 \\
\hline $16530 W 240 C C$ & -100.584 & 38.6421 \\
\hline 1693042901313 & -100.660 & 34.6304 \\
\hline $1152742 \mathrm{cccc}$ & -100.337 & 38.5534 \\
\hline $17521425 \mathrm{ccc}$ & -100.281 & 38.539 \\
\hline $17528 \mathrm{~N} 7 \mathrm{aAH}$ & -100.467 & 38.595 \\
\hline $115294366 \mathrm{AA}$ & -100.477 & 38.5377 \\
\hline I AS? $1184 C$ & -100.456 & 38.4885 \\
\hline 18529 4 40 AD & -100.523 & 38.5142 \\
\hline $1: 33042 A A A$ & -100.596 & 38.5241 \\
\hline LES3IWJIHCH & -100.199 & 38.6252 \\
\hline $165334 \pi \quad 7 C C C$ & -101.016 & 38.672 \\
\hline $165.53519 \mathrm{COO}$ & -101.015 & 38.5472 \\
\hline Ir:S3.5W:IHCC & -100.979 & 34.6512 \\
\hline $1<5334254 C C$ & -100.923 & 38.6370 \\
\hline 16534.J2c08C & -101.100 & 39.646 .5 \\
\hline $15 S 34$ W $2 A C C$ & $-101.06,7$ & 38.6505 \\
\hline $1 E S 34129 C$ BB⿴ & -101.109 & 38.6336 \\
\hline les34:434C BII & -101.071 & 38.619 \\
\hline 17031 प्र318НH & -100.797 & 38.5410 \\
\hline 115324 5A & -100.078 & 38.613 \\
\hline $11532416 \mathrm{HB11}$ & -100.469 & 38.5944 \\
\hline $17.5324318 \mathrm{Col}$ & -100.904 & 38.5368 \\
\hline $17532.13 \cap C A A$ & -100.1844 & 38.5334 \\
\hline $17533478 \mathrm{FH}$ & -101.015 & 38.5781 \\
\hline $17533+14 A C .11$ & -100.932 & 38.5130 \\
\hline $115.33 W 1 E A B C$ & -100.969 & 38.5820 \\
\hline 125344 fBCH & -101.127 & 38.6090 \\
\hline 17.354 CACOCO & -101.680 & 38.5793 \\
\hline $17534.22 \mathrm{nccl}$ & -101.089 & 38.542 \\
\hline $190.32119 \mathrm{nH}$ & -100.833 & 38.4759 \\
\hline 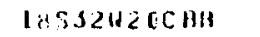 & $-100.13 B G_{6}$ & 38.474 \\
\hline $1053342 \mathrm{CCA}$ & -100.960 & 39.514 \\
\hline $14333450 \mathrm{cc}$ & -100.997 & 38.512 \\
\hline 1PS3 3 2. $2(0)$ & -100.994 & 38.468 \\
\hline 14534. TCWM & -101.034 & 38.5178 \\
\hline $145344258+0$ & -101.031 & 38.465 \\
\hline 14531436000 & -101.112 & 38.153 \\
\hline $10-.34+34380$ & -101.070 & 38.750 \\
\hline $1053142(1140$ & -100.770 & $3 n .392$ \\
\hline $19333+1501313$ & -100.948 & 38.3498 \\
\hline 1053SHCAAHII & -100.913 & 38.394 \\
\hline $155334250 C n$ & -100.910 & 38.3671 \\
\hline $19333: 4940 C$ & -100.950 & 38.352 \\
\hline $1993.3 \% \geqslant 30 \mathrm{Ba}$ & -100.996 & 311.3722 \\
\hline 2553111140110 & -100.721 & .94 .312 \\
\hline $20: 332 \mathrm{VCOA}$ & -100.901 & $38.32 n$ \\
\hline
\end{tabular}

ACTUAL DEGRES C

$$
\begin{aligned}
& \begin{array}{l}
16.0 \\
17.0
\end{array} \\
& 15.0 \\
& 14.5 \\
& 14.5 \\
& \begin{array}{l}
16.0 \\
16.0
\end{array} \\
& 16.0 \\
& 15.0 \\
& 16.0 \\
& 16.0 \\
& 16.0 \\
& 16.0 \\
& 17.0 \\
& 16.0 \\
& 15.0 \\
& 15.5 \\
& 18.5 \\
& 15.5 \\
& 16.5 \\
& 16.5 \\
& 16.0 \\
& 16.0 \\
& \begin{array}{l}
15.5 \\
15.5
\end{array} \\
& \begin{array}{l}
15.5 \\
16.0
\end{array} \\
& \begin{array}{l}
16.0 \\
15.5
\end{array} \\
& \begin{array}{l}
15.5 \\
15.5
\end{array} \\
& 16.0 \\
& 16.5 \\
& 16.0 \\
& 16.0 \\
& 16.0 \\
& 15.0 \\
& 17.0 \\
& 16.5 \\
& 17.0 \\
& 16.0 \\
& 17.0 \\
& 16.0 \\
& \begin{array}{l}
16.0 \\
17.0
\end{array} \\
& \begin{array}{l}
17.0 \\
17.0
\end{array} \\
& 16.0 \\
& 17.0 \\
& 15.0 \\
& 16.5 \\
& 18.0 \\
& 15.0 \\
& 16.0 \\
& 17.5 \\
& 15.5 \\
& 16.5 \\
& \begin{array}{l}
16.5 \\
14.0
\end{array}
\end{aligned}
$$

GE OTHERMOMETER TEMPERATURES - DEGREES C

OUARTZ CHALCEDONY NA-K

$N A-K-C A$

NA-K-CA LO

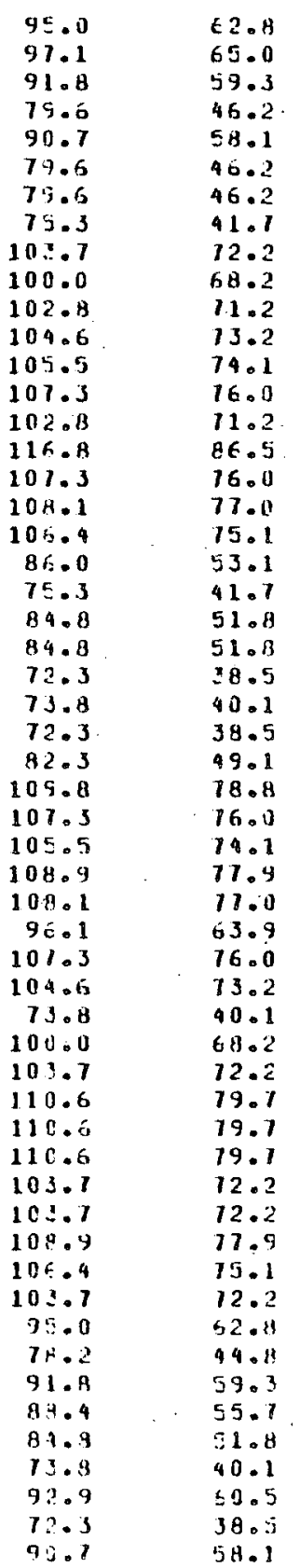

245.5

45.1

6.2

$\begin{array}{lll}225.8 & 48.8 & 9.2 \\ 222.7 & 41.4 & 5.8\end{array}$

$300.1 \quad 39.4 \quad 1.5$

$256.5 \quad 52.9 \quad 10.6$

$299.8 \quad 32.6 \quad 5.6$

$482.1 \quad 34.3 \quad 2.7$

$\begin{array}{lll}418.9 & 27.6 & -16.0\end{array}$

$330.244 .0 \quad 4.0$

$\begin{array}{rrr}308.6 & 96.0 & 5.8 \\ 299.5 & 19.4 & -2.4\end{array}$

$\begin{array}{lll}318.1 & 4.3 .3 & 1.1\end{array}$

$330.2 \quad 43.0 \quad 3.2$

$306.1 \quad 48.0 \quad 8.4$

$296.5 \quad 53.5 \quad 6.0$

$335.0 \quad 53.2 \quad 5.6$

$278.5 \quad 56.5 \quad H .2$

$49.5 \quad 43.9 \quad .9$

$26.3 \quad 47.1-8.3$

259.8 (18.5

$250.0 \quad-1.4$

$\begin{array}{lll}250.0 & 48.4 & -1.4 \\ 254.0 & 49.4 & -3.6\end{array}$

$299.0 \quad 47.8 \quad-5.2$

$\begin{array}{lll}251.9 & 47.9 & 2.8\end{array}$

$\begin{array}{lll}243.9 & 51.4 & 4.5 \\ 300.6 & 51.1 & 5.5\end{array}$

$\begin{array}{lll}300.6 & 51.1 & 5.5 \\ 256.9 & 48.2 & 3.3\end{array}$

479.3
276.5

$276.6 \quad 50.5 \quad 1.1$

296.651 .80

$254.0 \quad 61.2 \quad 9.9$

$256.4 \quad 54.2 \quad 5.6$

$247.2 \quad 48.7 \quad 7.9$

251.50

$\begin{array}{rrr}276.6 & 54.6 & 13.9 \\ 276.6 & 51.7 & 4.7\end{array}$

$\begin{array}{lll}276.6 & 51.7 & 4.7 \\ 264.5 & 56.9 & 8.6\end{array}$

$266.251 .5 \quad 06$

$294.6 \quad 50.2 \quad-2.6$

$307.3 \quad 47.0 \quad 1.7$

$278.9 \quad 53.0 \quad 8.9$

$281.7 \quad 45.9 \quad-.9$

$305.6 \quad 54.6 \quad 4.4$

$303.3 \quad 52.8 \quad 3.8$

$190.5 \quad 63.1 \quad 11.5$

$258.0 \quad 57.2 \quad 6.2$

$322.8 \quad 52.8 \quad 4.6$

$290.8 \quad 53.8$

$\begin{array}{lll}274.3 & 65.5 & 12.2 \\ 253.1 & 66.5 & 11.8\end{array}$ 
WELL LOCATION

\begin{tabular}{|c|c|c|}
\hline ANSHIP-RANBE & LONGI TUCE & LATITUDE. \\
\hline 20532116010 & -100.851 & 36.3123 \\
\hline $20532 \omega: 1$ atc & -100.367 & 38.3050 \\
\hline $2 n 5334$ 20519 & -100.931 & 38.3433 \\
\hline $20533 \because 1$ COFC & -100.450 & 38.3268 \\
\hline 20S3SWIZHAH & -100.991 & 39.3212 \\
\hline 20335421 A (41) & -100.766 & $3 \mathrm{H} .3049$ \\
\hline 20533N35011 & -100.928 & 38.2103 \\
\hline $20.34 \mathrm{~W}$ CDEC & -101.042 & 38.3411 \\
\hline $14540 \% 23 \mathrm{CEO}$ & -101.726 & 38.01225 \\
\hline 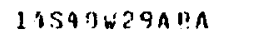 & -101.787 & 38.3130 \\
\hline 145414220013 & -101.872 & 38.8269 \\
\hline 14512614000 & -101.952 & 30.8317 \\
\hline 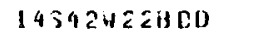 & -101.975 & 38.8206 \\
\hline 14342.220191 & -101.970 & $3 B .81 A B$ \\
\hline 1А5А2W $О C B C A$ & -102.034 & 38.8075 \\
\hline $15533 \mathrm{~W} 73 \mathrm{HH}$ & -101.594 & 38.7710 \\
\hline 16. 35 BS:1 $4 C E O$ & -101.518 & 38.7431 \\
\hline issunjeccus & -111.596 & 38.7168 \\
\hline $15738136 \mathrm{C}$ BII & -101.503 & 38.7059 \\
\hline $155392 \quad 2 \mathrm{ACO}$ & -101.629 & 38.7797 \\
\hline $15539 H \mathrm{H}$ CCAA & -101.704 & 38.7174 \\
\hline 15537 A BACC & $-101.6,71$ & 38.1650 \\
\hline 159394263014 & -101.633 & 38.1273 \\
\hline $155 \% 3 \mathrm{~d} 7 \mathrm{nBn}$ & $-101 \cdot 418$ & 38.7693 \\
\hline 1 SUAU1 $20 A A$ & $-101.71 \mathrm{~A}$ & 38.7556 \\
\hline $15 S 4042$ CORH & -101.889 & 38.7186 \\
\hline 159414 EAC:3 & -101.900 & 38.7794 \\
\hline IESA1HIOHAH & -101.868 & 38.7688 \\
\hline $1534 \mathrm{tW} 27 \mathrm{CBC}$ & -101.874 & 88.7161 \\
\hline IГSA?H EBHBH & -101.964 & 38.7825 \\
\hline 1 1SS42W32ACA & -102.013 & 38.7056 \\
\hline $16533 H 2 c 0 c 0$ & -101.218 & 38.6423 \\
\hline ICS5JИ27HAC & -101.192 & 38.6397 \\
\hline 16555H.J10HA & -101.225 & 38.5136 \\
\hline 16335.121000 & -101.310 & 38.6425 \\
\hline 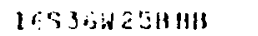 & $-101 \cdot 255$ & 38.6405 \\
\hline 16535434000 & -101.292 & 38.6134 \\
\hline $18336+250 C$ & -101.273 & $3 R .5133$ \\
\hline 10.351360110 & -101.255 & 38.06169 \\
\hline $16537 \mathrm{~A} 90 \mathrm{CC}$ & -101.912 & 34.6351 \\
\hline iecs7atecce & -101.402 & 38.5570 \\
\hline 160374171304 & -101.439 & 38.6697 \\
\hline ifSSIU27ALI: & -101.393 & 38.6389 \\
\hline 1 fos $182 \operatorname{sinlta}$ & -101.139 & 38.6 .406 \\
\hline $16.931: 13 \mathrm{CACH}$ & -101.448 & 38.6369 \\
\hline lecsous Enata & -101.549 & .38 .5937 \\
\hline 1633\%:1CABH & -101.503 & $36.644 ?$ \\
\hline $16539416 A C C$ & -101.522 & 39.6692 \\
\hline 15958125B34" & -101.494 & $3 H .5405$ \\
\hline 16:34:29ACH & -101.535 & .35 .6569 \\
\hline 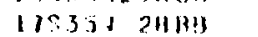 & -101.164 & 38.6115 \\
\hline 1833415000 & -101.111 & 31.5697 \\
\hline $17335 \mathrm{dI} 44 \mathrm{CH}$ & -101.227 & 38.5787 \\
\hline $173391270 \mathrm{cc}$ & -101.191 & 38.5407 \\
\hline i 1536 seco & -101.326 & 31.03949 \\
\hline
\end{tabular}

ACTUAL

TEMP

DF. GREES C

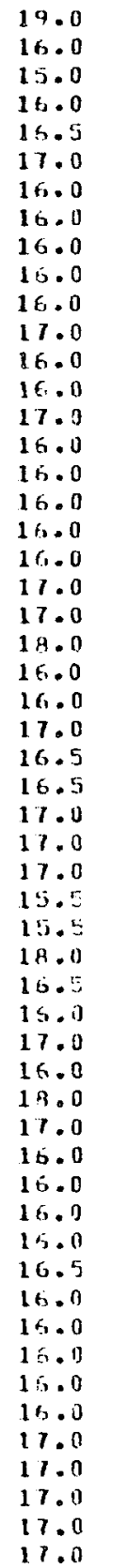

Ge UTHERMOMETER temperatures - DEgrles C

QUARTZ CHALCEDONY

$N \wedge-K$

NA-K-CA NA-K-CA LO

79.5

46.2

332.1

49.5

95.0

$42 . \mathrm{A}$

43.3

47.7

50.5

83.6

89.6

86.0

$78 \cdot 2$
$5.3 \cdot 2$

$5: 3$

61.3

50.8

55.8

67.5

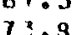

73.8
73.2

70.7

$7 \mathrm{C} .7$
$7 \mathrm{E} .8$

70.7

6.5 .5

72.3

72.3
$6 \because .7$

$6: .7$
65.5

70.7

6.5 .5

65.5

70.7

63.1

67.3

70.1

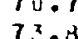

30.8
73.4

70.7

70.7
69.0

69.0
72.3

72.3
70.7

$7 \leq .3$

72.3

72.3

$7 \mathrm{C} .7$

6. 0

1... 3

70.7
70.7

70.7
70.7

70.7

7.01

$8+6$

72.3
103.0

$91: 0$

101.0
73.8

53.1
44.8

44.8
18.6

21.0

27.5

25.4

25.8

21.0

33.3

40.1

44.33
36.3

36.9

43.3
36.3

36.8
31.4

31.4
38.5

38.5

29.5

31.1
36.8

31.4

31.4

36.01

29.5

33.3

36.0

36.8
40.1

$36 . ?$

36.8

35.1
38.5

36.4

41.1

38.5

38.5

36.3

3.1
30.5

36.3

36.13
36.8

36.8

35.8
43.3

56.7

46.7
38.5

60.?

62.

59.2
10.1

202.1

216.6

$2 A 7.1$

328.5

342.4

413

269.2

266.0

255.0

252.9

251.1

241.0

2á8.0

237.0

271.1

271.1

263.6

242.0

245.0

251.0

264.6

270.4

250.8

276.0

267.7

243.0

254.8

276.6

276.6

259.1

2419

246.5

246.5

255.5

249.1
256.2

256.2

227.9

260.9

257.5

244.9

251.?

242.6

273.7

265.1

255.4

292.

261.4

240.0

269.4

266.9

264.8
256.2

13.1

$53.4 \quad 4.6$

$53.5 \quad 4.8$

$52.8 \quad 4.7$

$49.9 \quad 4.9$

$43.8 \quad-1.6$

$42.4 \quad .7$

$42.9 \quad-.3$

$46.7 \quad 5.0$

$45.2 \quad 3.9$

$47.2 \quad 3.6$

$45.1 \quad-1.6$

$52.1 \quad-9.4$

$\begin{array}{lr}\text { TE. } & 7.9 \\ \text { A } & -1.5\end{array}$

$48.9 \quad-.5$

$\begin{array}{lr}49.6 & 1.0 \\ 50.1 & .0\end{array}$

$49.7 \quad-.3$

50.12 .9

$\begin{array}{ll}48.3 & -1.1 \\ 61.1 & 9.9\end{array}$

$48.4 \quad 3.5$

$50.2 \quad 4.6$

$51.1 \quad 5.0$

$53.8 \quad 7.2$

$47.6-4.1$

$47.5 \quad-5.3$

$48.0 \quad-7.7$

$\begin{array}{ll}91.3 & 5.5 \\ 47.5 & 2.7\end{array}$

$52.3 \quad 7.0$

$52.1 \quad 5.0$

$\begin{array}{ll}51.2 & 5.1 \\ 49.7 & 3.9\end{array}$

$5 \mathrm{c.5} \quad 4.9$

$51.4 \quad 5.8$

$54.1 \quad 0.5$

56.5

$50.8 \quad 5.2$

134.1

$52.9 \quad 1.1$

$51.4 \quad 5.8$

48.8

52.1

58.6

51.6

9.8

11.8

13.5 
WELL LOCATIOH

TOLNSHIF-RANGE LONGITUDE LAIITUDE

ACTUAL

IEMP

DEGREES C

$-101.292$

$-101.389$

$17335,10 C \mathrm{BH}$

IDSTI ZAAH

11337in 3000

$115314226 \mathrm{CC}$

$17337424 C C C$

1133 ?HICAAII

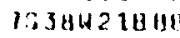

TS3AH2 $4 A C C$
IS3 3 H 1 IACO

$1753842 \mathrm{BCCC}$

1PSSOSA $2 \mathrm{ACH}$

$19335 \mathrm{~N}$ 8H PCC

$185354170 \mathrm{Aa}$

IPS35W34AAB

PS3r' $20 \mathrm{CH}$

B536. $3131 ! 13$

$18536 W 1$ EOAD

$19536429 A B 13$

19S3TA ICCC

AS 37 II 1 BAAD

PS37H21HPH

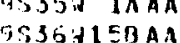

1 9\$3 TW2 2AAB

$15538 H 14 A A B$

15539:180CC

$15 \triangle 3 B 426 C C H$

ISS3BW $3511 A 4$

$2053 \mathrm{H} 11 \mathrm{COB}$

21331 HECCC

$21332 \% 8 A B D$

IS.32 प्रश्C CBD

$21533 \mathrm{H} 2 \mathrm{ACB}$
$21333 \mathrm{H} 7011 \mathrm{~A}$
$2133 \mathrm{~W} 2 \mathrm{CAA}$

$21333425 C A A$

$21533121 \mathrm{CHA}$

$22531 \mathrm{~W}$ inCC

2 CS31U1 $20 \mathrm{CC}$

$255324210 \mathrm{CO}$

$22512: 55 \mathrm{BH}$

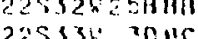

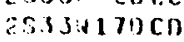

22933+22HAA

$22533432 \mathrm{CHC}$

2S33:2 IEA AA

22514.1 AHCA
$\because 034131$ CACD

$\because C 34161$ CACD

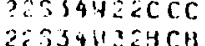

c.saJWJ4C0?

2ess1W $30 \mathrm{Col}$

a:ST1H 30111

?.5311.9ECCC

\section{$3 B .3497$}

$3 B .6116$

38.5970

38.569

38.5553
30.5407

39.5407
38.5969

3B. 5 Fit

38.5224

39.5918

38.5405

38.5207

38.5379

38.5379

38.4840

38.4516

38.5134

38.5099

38.4462

38.4663

3月. 5116

38.4788

38.4907

38.4372

38.4081

38.3936

38.4079

38.3951

38.3679

38.3542

38.31242

38.3115

38.1920

38.2108

$38 . ? 107$

311.25994

30.2376
38.1981

36.1981
38.1834

B.1 4 88

38.1495

$3+.1034$

38.1204

39.1194

38.1674

38.1348

38.134

36.1331

38.0351

38.1044

38.1578

38.15652

33.1200

38.0079

38.0033

38.0763

$3 \mathrm{H} .0674$

38.0039

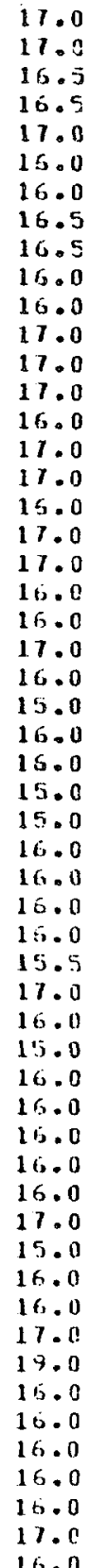

16.5

7.0

26.0

16.5

16.5
16.0
16.0

16.0

17.0

I. 0

5.0

17.0

16.0

16.0

5.0

5.0

15.0
16.0

16.0

5. 0

15.5

60

16.0
15.0
16.0

6.0

15.0

6.0

16.0

5.0

16.0

17.0
19.0

15.0

6.0

th.
17.0
16.0
82. 3

B日. 4

80.6
$10 \mathrm{C} .0$

101.3

91.8

105.5

105.5
94.0
98.1

$9<.1$

108.3

$10 \% \cdot 3$

102.8

95.1
101.0
101.9

101.9
100.0

$9 \dot{0.1}$

94.0

96.1

$8 \% .4$

B. 6

37.1

101.9

91.9

96.1

101.0

99.1

$7 \in .8$

113.7

115.3

118.2

95.0

101.9

104.6

47.3

106.9

112.2

100.4

87.3

950

83.15

70.7
70.7

7!. 1

73.8

79.7

72.3

$6 ? .7$

101.9

101.9
101.9

6?. 3

95.0

GE OIHERMOMETER TEMPERATURES - DEGREES C

CHALCEDONY NA-K NA-K-CA NA-K-CAL?

49.1

55.1

55.9

68.2
70.2

59.3

74.1

61.6
63.9

55.0

76.0

71.2

67.1

69.2
70.2

68.2

63.9

61.6

55.7

56.9

65.0

66.1

10.2
63.9

63.9

63.9

69.2
66.1

66.1
43.3

स. 3.1

84.8

28.1

62.8

70.2

73.2

34.4

81.4

15.1

54.4

$52 \cdot 8$

50.5
36.8

36.8

36.9

40.1

36.9

3 . .5

29.5

29.5

70.2

3.3
02.9

$\begin{array}{ll}255.7 & 50.7 \\ 245.7 & 52.1\end{array}$

$289.6 \quad 47.0$

$251.7 \quad 52.9$

$24 \mathrm{~B}_{0.4}$

239.5

263.6

246.5

276.6

276.5

276.6

266.6

273.8

271.5

280.4

261.9

342.4

335.2

316.1

286.9

250.8

265.9

266.6

282.5

251.7

212.2

151.3

172.9

170.7

223.1

187.5

232.9

2650

265.5

199.5

190.5 
WELL LOCATION

\begin{tabular}{|c|c|c|}
\hline OWNSHIP-HANSE & LONGITUDE & LAI \\
\hline $225321140[0.1]$ & -100.829 & 38.07 \\
\hline $2: 532 W 11 \mathrm{ADC}$ & -100.795 & 38.0697 \\
\hline $23532418 H C 0$ & -100.879 & 98.0556 \\
\hline $233320200 \mathrm{A13}$ & -100.813 & 38.0341 \\
\hline $2.3532431 \mathrm{CA}$ & -100.375 & $39.00,96$ \\
\hline $23.333 \mathrm{WlCOCr}$ & -100.327 & 38.0528 \\
\hline 27833417963 & -100.973 & 38.0609 \\
\hline 28533.6269111 & -100.908 & 38.0321 \\
\hline $2.53 .5 \mathrm{H} 29 \mathrm{CCC}$ & -100.950 & 38.0194 \\
\hline $2: 3.3 \mathrm{SW} 32 \mathrm{ABO}$ & -100.964 & 38.0176 \\
\hline $2 \geq \subseteq 33 W \Omega \subseteq A C C$ & -100.9118 & $3 R .0123$ \\
\hline 22534t 3HCN & -101.047 & 38.0857 \\
\hline 28534114000 & -101.024 & 38.0552 \\
\hline $2: 534617 \mathrm{CcC}$ & -101.084 & $3 R .0475$ \\
\hline $24.54 \times 21000$ & -101.051 & 38.0333 \\
\hline $2 \times 53+1280 \mathrm{gCA}$ & -101.058 & 38.0206 \\
\hline 29.331411080 & -100.688 & 37.9785 \\
\hline $24531: 17000$ & -100.738 & 37.9609 \\
\hline 24S3?: 5BCB & -100.862 & 37.9996 \\
\hline $29532425 \mathrm{C} 83$ & -100.790 & 31.9379 \\
\hline $20532 \mathrm{~V} 29 \mathrm{AC}$ & -100.852 & 37.9409 \\
\hline $21533 ! 1148383$ & $-100 \cdot 917$ & 37.9744 \\
\hline $24333 \mathrm{~b}: 2 \mathrm{iCA11}$ & -100.949 & 37.9527 \\
\hline $243.33 W 220 C A$ & $-10 n .924$ & 37.9491 \\
\hline $24: 333112010 A$ & $-100 \cdot 338$ & 31.9383 \\
\hline $25033435 \mathrm{CCA}$ & $-100 \cdot 914$ & 31.8337 \\
\hline $255341611 A 0$ & -101.095 & 37.9135 \\
\hline $255371110 \mathrm{Cn}$ & -101.012 & 37.8892 \\
\hline $255344 \geq 4000$ & -101.034 & 37.834 .9 \\
\hline 265314 GHFC & $-100 \cdot 160$ & 37.8276 \\
\hline 26331431000 & -100.156 & 31.1429 \\
\hline $2 E S 3 \perp W B E C A C$ & $-100,6,6,3$ & 37.7455 \\
\hline $2 E 532: 22 A ! 1 B$ & -100.805 & 37.7855 \\
\hline $2+532435 C 0 A$ & -100.790 & 37.7949 \\
\hline $26533630 \mathrm{HHH}$ & -100.914 & 37.8227 \\
\hline $2.535 w 1230 c$ & $-100 \cdot B B 2$ & 37.3098 \\
\hline 26SЗSH?EAHY & -100.896 & 37.7711 \\
\hline $21935.117 \mathrm{ACN}$ & $-101 \cdot 16.1$ & 37.7057 \\
\hline $27535 \% 34 B B A$ & -101.139 & 31.6570 \\
\hline 279351116000 & -101.234 & 37.6595 \\
\hline $27336.1160 \mathrm{CH}$ & -101.295 & 37.1001 \\
\hline E153/4 $4 \mathrm{All}$ & -101.369 & 37.7102 \\
\hline $27537 W 1 \mid A B A$ & -101.330 & 37.7256 \\
\hline 21537422 AH: & $-101 \cdot 360$ & $37.60 \mathrm{~F} 2$ \\
\hline $2153 r 12 \mathrm{eHCH}$ & $-101 \cdot 341$ & 31.6179 \\
\hline $21531: 192900 !$ & -111.396 & 31.6741 \\
\hline 215SAUE2CHI & -101.470 & 31.6985 \\
\hline 2 $15344<5 n \mid 11$ & -101.433 & 31.6413 \\
\hline $2 ! C 3 A 1 S 2 H B C$ & -101.200 & $32.66,46$ \\
\hline $29527 ! \mathrm{HCCL}$ & -100.317 & 31.9140 \\
\hline ? $4324410 A C N$ & $-100 \cdot 374$ & 37.9914 \\
\hline $2452890 \div B \| B ! A$ & -100.406 & 37.9436 \\
\hline $2452+13100$ & -100.431 & 37.9175 \\
\hline Q1729WLECA & -100.507 & 7.7318 \\
\hline$? 432+164 C L B$ & -100.554 & 37.9618 \\
\hline
\end{tabular}

ACTUAL

DEGREE.S C
GEOTHERMOMETER TEMPERATURES - DEGREES C

QUARTZ CHALCEDONY NA-K

as.

87.

$7 \geq$.

76.8

65.0

73.7

70.7

73.8

72.3

$7 \% .2$

$7 \% .2$
70.7

70.8
73.8

61.3

$7: .6$

79.2
94.0

94.0

70.7

70.7
67.3

61.8

61.8

61.8

68.8

51.7
6.27

57.7

57.7

57.7

$5 \div 03$

61.8

63.8

57.7

51.8

31.8

61.9

$5=2$

61.8

$5 \mathrm{C.}$

59.8
7.2

79.2
6.9 .0

72.3

79.2

70.7

70.7

70.1

6.9

91.0

95.1

97.1

94.0
$9 ? .9$

95.0

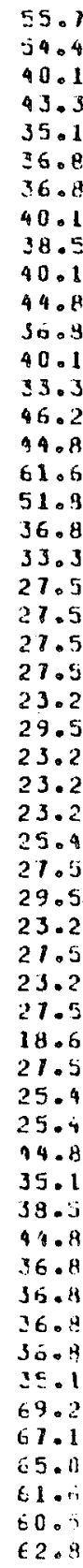

197.8

204.0

206.6

$231 . A$

139.6

212.9

222.8

172.5
107.1

107.1
128.1

98.9

251.9

179.1

189.0

$152 . A$

210.4

196.7

145.5

107.6

140.6

140.6

228.1

198.1
216.3

216.3
260.9

215.0

219.1

306.1

248.8

210.7

226.9

219.5

199.6

217.6

283.5

256.9

199.2
150.1

150.4
177.6

166.9

209.1

134.5

169.0

194.6

182.8
294.6

305.5

276.5

276.6

$28 \mathrm{~B} .2$

334.2
290.6
NA-K-CA

\begin{tabular}{|c|c|}
\hline $\begin{array}{l}46.8 \\
44.0\end{array}$ & $\begin{array}{l}3.8 \\
3.3\end{array}$ \\
\hline 44.8 & 6.9 \\
\hline 11.9 & 4.9 \\
\hline 47.0 & 3.2 \\
\hline 45.7 & 3.4 \\
\hline 40.8 & 3.5 \\
\hline 49.6 & 1.7 \\
\hline 58.1 & 16.7 \\
\hline 53.6 & 13.0 \\
\hline 61.0 & 8.9 \\
\hline 40.1 & .8 \\
\hline 44.8 & b. 2 \\
\hline 36.4 & -3.6. \\
\hline 41.9 & 5.2 \\
\hline 53.2 & 10.7 \\
\hline 195.4 & 1.0 \\
\hline 45.5 & 2.6 \\
\hline 56.7 & 8.7 \\
\hline 60.5 & 6.3 \\
\hline 18.7 & -15.3 \\
\hline 90.6 & 3.4 \\
\hline $2 \mathrm{~B} .4$ & $-\because 1$ \\
\hline 29.4 & -7.3 \\
\hline 27.8 & -8.7 \\
\hline 23.3 & -12.5 \\
\hline 26.6 & -4.2 \\
\hline 25.2 & -9.7 \\
\hline 19.6 & -12.9 \\
\hline 24.9 & $-11 \cdot 3$ \\
\hline 26.6 & -8.9 \\
\hline 27.9 & -7.8 \\
\hline 26.4 & $-10 \cdot 6$ \\
\hline 24.7 & -12.1 \\
\hline 23.3 & -11.6 \\
\hline 25.2 & -11.1 \\
\hline 19.1 & -11.2 \\
\hline 18.7 & -11.5 \\
\hline 18.0 & -16.00 \\
\hline 32.0 & -5.0 \\
\hline 45.3 & 1.1 \\
\hline $5 . .3$ & 7.5 \\
\hline $50 . ?$ & 10.3 \\
\hline 31.5 & $-1 \cdot 6$ \\
\hline 45.1 & 5.0 \\
\hline 35.9 & 1.8 \\
\hline 39.0 & -.2 \\
\hline 35.9 & -3.3 \\
\hline 39.1 & $1 . A$ \\
\hline 41.8 & 6.2 \\
\hline 44.5 & 5.0 \\
\hline 16.5 & 1.0 \\
\hline 42.6 & 4.5 \\
\hline 41.6 & 5.1 \\
\hline 44.9 & t. . 9 \\
\hline
\end{tabular}


\ELL LOCATION

\begin{tabular}{|c|c|c|}
\hline TOANSHIF-RANIIE & LUNGI TUOE. & LATITUN \\
\hline $24529424 A C D$ & -100.448 & 37.952 \\
\hline $29530 \mathrm{~d} 13 \mathrm{CH}$ & -100.572 & 51.9979 \\
\hline 243304 ACCO & -100.634 & 37.3746 \\
\hline $245304150 \mathrm{CC}$ & -100.609 & 37.9601 \\
\hline $24530131 \cap 41$ & -100.654 & 37.9294 \\
\hline $2552 \operatorname{lnl} 1 \mathrm{CCCC}$ & -100.336 & 37.8588 \\
\hline $2.5271334 H H$ & -100.290 & 37.8423 \\
\hline $25 \operatorname{s.2} 7 ! 13 \leq 000$ & $-1 \cup 0.258$ & 37.4295 \\
\hline $2592 B \div 16 B \mid 34$ & -100.409 & 37.8859 \\
\hline $2 \leqslant 52 A 131 H H C$ & -100.446 & 37.8409 \\
\hline $25: 52947400$ & -100.554 & 37.9958 \\
\hline $2 \leq 329+14 A B B$ & -100.473 & 37.9860 \\
\hline $25 \leq 294331111 \mathrm{C}$ & -100.318 & 37.9408 \\
\hline 2 ES304 2 CACH & -100.645 & 37.8678 \\
\hline $2 \sin 7 \times 13 a 11 \mathrm{C}$ & -100.233 & 37.7963 \\
\hline 2652711 AADC & -100.310 & 37.1930 \\
\hline $2 t: \$ 2 / W 27 C 00$ & -100.262 & 37.7559 \\
\hline 2ES? HWI9AHB & -100.421 & 37.7823 \\
\hline 2.5313! 1CDA & -100.553 & 37.8171 \\
\hline $2 E S 30417 A 0$ & -100.517 & 37.7941 \\
\hline cEs $39 \pi 24000$ & -100.544 & 37.7712 \\
\hline $2 \operatorname{sis} 30428 A C !$ & -100.598 & 37.7638 \\
\hline $27527 \mathrm{~L} \in \mathrm{BBH}$ & -100.323 & 37.1397 \\
\hline 27S27L TACC & -100.310 & 37.7193 \\
\hline $27527 \times 19880$ & -100.322 & 37.6936 \\
\hline $2152 ! 113 C C C A$ & -100.930 & 37.5101 \\
\hline $27329.290 \mathrm{~A}$ & -100.491 & 37.7170 \\
\hline $27323: 100 \mathrm{H}$ & -100.533 & 37.7032 \\
\hline 275294Z3AกC & -100.455 & 37.6904 \\
\hline $27: 323 \times 33 \mathrm{CCC}$ & -100.506 & 37.5536 \\
\hline$\therefore \cos 3 \mathrm{QN}$ คВН & -100.631 & 37.1242 \\
\hline $23530425 \mathrm{CCB}$ & -100.560 & 37.5701 \\
\hline $21530434 C \mathrm{CC}$ & -100.596 & 37.6596 \\
\hline 28530W 6HBA & -100.648 & 37.69 .97 \\
\hline $23942119 \mathrm{CBO}$ & $-101.3 \times 1$ & 38.0374 \\
\hline 235422270011 & -101.912 & 38.0174 \\
\hline 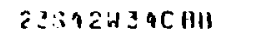 & -101.926 & 38.0045 \\
\hline $23343 \times 25 C B O$ & -101.997 & 38.0210 \\
\hline $245394190 \mathrm{BC}$ & $-101.65 n$ & 37.9492 \\
\hline $24539 W 3 \mathrm{CHAO}$ & -101.648 & 37.7410 \\
\hline $24539+30 C A D$ & -101.643 & 37.9338 \\
\hline $24.340417 \mathrm{H} 134$ & -131.742 & 37.9720 \\
\hline $25043: 426001)$ & -102.002 & 37.8439 \\
\hline EASA1U2OHAD & -101.831 & 37.7815 \\
\hline $25341: 36 \mathrm{CC}$ & -101.159 & 37.7419 \\
\hline 25842210411 & $-101.00 \mathrm{~A}$ & 37.9119 \\
\hline 2054241700 & -101.941 & 37.7899 \\
\hline $2551 \geq 42 z \mathrm{Cr} H$ & -101.901 & 37.7125 \\
\hline ZSS3 BAOH & $-10 ? .03 A$ & 37.8091 \\
\hline $26 \$ 43 H 2 S 0 C C$ & -101.969 & 37.7560 \\
\hline $27391: 130104$ & -100.753 & 31.1226 \\
\hline 21531 W 40 B. & -100.664 & 37.19 .930 \\
\hline $27532 \mathrm{~W} \quad$ 5C HII & $-100.86 ?$ & 57.7543 \\
\hline $215341190 \mathrm{co}$ & -100.866 & 37.6945 \\
\hline$=15.334=7 C \cap 0$ & -160.916 & 37.6135 \\
\hline
\end{tabular}

ACIUAL

TEMP

DEGREES C

16.0
16.0
16.0
16.0
16.0
17.5
17.0
18.0
16.5
16.0
16.0
16.5
16.0
15.0
16.0
15.5
16.0
17.0
17.0
16.0
16.0
16.0
11.0
16.5
16.5
17.0
17.0
16.0
16.5
17.0
16.0
16.0
16.5
11.0
15.5
16.0
15.0
14.5
14.5
19.5
15.0
15.0
19.0
16.0
17.1
13.0
11.0
11.0
17.0
11.0
15.5
15.5
19.5
17.5
19.1
1
19

GEOTHERMOMETER TEMPERATURES - OEGREES C

QUARTL CHALCCDONY NA-K NA-K-CA NA-K-CA LO

\begin{tabular}{|c|c|}
\hline 95.0 & o 2.3 \\
\hline $10 f: 04$ & 75.1 \\
\hline 97.1 & 65.8 \\
\hline 91.8 & 59.3 \\
\hline 82.3 & 49.1 \\
\hline 83.7 & 55.7 \\
\hline $8+.1$ & 55.7 \\
\hline 87.3 & 54.4 \\
\hline 90.7 & 58.1 \\
\hline 72.3 & 38.5 \\
\hline 79.5 & 46.2 \\
\hline $7 ! 1.2$ & 44.3 \\
\hline 61.9 & 27.5 \\
\hline 50.8 & 16.1 \\
\hline 63.7 & 29.5 \\
\hline 63.7 & 29.5 \\
\hline 65.5 & 31.4 \\
\hline $6: .1$ & 29.5 \\
\hline 59.8 & 25.7 \\
\hline 51.7 & $2 \pi .2$ \\
\hline 63.7 & 29.5 \\
\hline 58.7 & 23.2 \\
\hline 67.3 & 33.3 \\
\hline 65.5 & 31.4 \\
\hline 63.7 & 29.5 \\
\hline 63.7 & 3.5. \\
\hline 59.4 & 25.4 \\
\hline 61.8 & 27.5 \\
\hline 59.8 & 25.4 \\
\hline 63.1 & 29.5 \\
\hline 63.7 & 29.5 \\
\hline 51.7 & 23.2 \\
\hline 59.8 & 25.4 \\
\hline $5=.5$ & 21.0 \\
\hline 80.9 & $\$ 7.7$ \\
\hline 56.5 & 21.0 \\
\hline 45.6 & 10.1 \\
\hline 50.8 & 16.1 \\
\hline $51 . .4$ & 16.1 \\
\hline 49.3 & 13.0 \\
\hline 57.1 & 23.2 \\
\hline 55.8 & 25.4 \\
\hline 49.3 & 13.3 \\
\hline $6 E .5$ & $21 . n$ \\
\hline 76.3 & 43.3 \\
\hline 35.6 & 4.1 \\
\hline 51.3 & 16.1 \\
\hline 50.9 & 16.1 \\
\hline 45.6 & 10.1 \\
\hline 511.8 & 16.1 \\
\hline 53.9 & 25.0 \\
\hline 5.9 & 25.4 \\
\hline $5 \% .7$ & 23.2 \\
\hline 31.7 & 23.2 \\
\hline 55.8 & 25 \\
\hline
\end{tabular}

304.7

273.9

$236 \cdot \mathrm{A}$

233.1

235.8

354.9

369.5

316.3

265.1

235.8

230.5

102.8

242.0

239.7

242.9

29.0

216.3

251.5

246.0

231.4

276.6
272.6

272.6
276.6

276.6

251.3

242.0

276.6

272.1

193.1

235.3

226.9

235.8

21.8

70.2
65.2

44.6

60.5

66.6

56.3

61.5

264.9

188.8

2013.4

239.7

266.0

262.9

301.3

241.0

213.7
251.7

211.5

$24 \mathrm{H} .3$
226.13

\begin{tabular}{|c|c|}
\hline 12.2 & 4.2 \\
\hline 46.0 & $\therefore .3$ \\
\hline 44.6 & 9.1 \\
\hline 46.2 & $B . b$ \\
\hline 39.1 & .9 \\
\hline 37.3 & -3.3 \\
\hline 31.4 & $\cdot 3$ \\
\hline 36.2. & $-2 \cdot 1$ \\
\hline 39.9 & 2.3 \\
\hline 34.6 & $-1 \cdot 5$ \\
\hline $3 H .1$ & . B \\
\hline 41.0 & .2 \\
\hline 29.9 & -7.9 \\
\hline $4 \% .1$ & 2.0 \\
\hline 31.1 & -3.2 \\
\hline 28.7 & -5.1 \\
\hline 35.9 & -2.5 \\
\hline 30.8 & -5.6 \\
\hline 21.2 & $-\xi .0$ \\
\hline $2 H .1$ & -7.8 \\
\hline 29.0 & $-0,05$ \\
\hline 26.2 & -6.2 \\
\hline 31.2 & -6.3 \\
\hline 30.0 & -9.2 \\
\hline 32.3 & -4.9 \\
\hline 30.7 & $-3 \cdot 0$ \\
\hline 32.9 & -5.1 \\
\hline 29.9 & -7.6 \\
\hline 31.4 & -6.4 \\
\hline 21.2 & -10.1 \\
\hline 29.0 & $-10 \cdot A$ \\
\hline 23.9 & -9.7 \\
\hline 22.5 & -11.4 \\
\hline 23.9 & -9.3 \\
\hline 15.7 & 2.1 .4 \\
\hline 67.7 & 19.3 \\
\hline 51.9 & 12.7 \\
\hline 64.3 & 1487 \\
\hline 54.6 & 101 \\
\hline 58.7 & 12.4 \\
\hline 66.1 & 14.4 \\
\hline 51.4 & 1.1 \\
\hline 32.5 & -3.4 \\
\hline 13.2 & -1.2 \\
\hline 43.1 & 1.5 \\
\hline 34.1 & -5.4 \\
\hline $3 ? .1$ & -4.0 \\
\hline 32.9 & -4.8 \\
\hline 33.0 & -1.1 \\
\hline $3 n .1$ & -1.5 \\
\hline 22.5 & $-12 \cdot 3$ \\
\hline 21.9 & -12.6 \\
\hline 21.9 & -10.0 \\
\hline 19.4 & -12.1 \\
\hline 20.3 & -11.2 \\
\hline
\end{tabular}


JELL LOCATION TOYNSHIP-PANGE LONGITUDE

$-100.944$ $-1011.930$ -1010.930
-101.035

$285336250 A A$ $21034.4160 \mathrm{CD}$ Els34it:zora $27.341280 \mathrm{~A}$ $22535 \cdot 23 \mathrm{Con}$

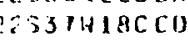
$28 C 35$ A $5 A C C$ $23535412 C C C$ 2.335625834 235364 4C 613 $205354 \div 213313$ 2 ISSIW19HCC $2.537 \mathrm{WHCCH}$ $24535413 \mathrm{CCO}$ $2453.5422 \mathrm{CCC}$ $24536+1511 C 11$ $29336 \mathrm{~d}^{2} 3 \mathrm{CHA}$ $24537 \mathrm{~W} 4 \mathrm{CCD}$ 5. 335 S2CAA $253361110 \mathrm{COC}$ $25336418 A C C$ 25s36.4194n! 2633642 gaH! $26535 \mathrm{~W}$ वBBO 26S35HM10CA 26S37H210[1) $2557+2600 C$ 1HS32.12CC R! 10.3SSH 35AAA $1 C 533 \mathrm{H} 120 D C$ $19533 \times 150 \mathrm{HD}$ 今5334 C5DCO $19333429 \mathrm{CH}$ $15533 \mathrm{H}=10 \mathrm{Cr}$ $20033 \% 20 \mathrm{HB}$ $20539+2141 ! n$ $20533426 C B n$ ? 1333 a 350114 $2 C 333 ! 2 ? C C D$ c7.39 vasaCo

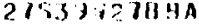
6790 W I SOLC 215406350135 $27540 \mathrm{~A}=\mathrm{BCC}$ 21511 il $1811 A n$ 21541 W $35 C \mathrm{CC}$ 2151? $31100 \%$ 37421700 $21 \cdot 12$ is $310 \mathrm{cr}$ 1343.12 aHCC 1 S5:30:1 4A AII

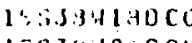
$175342560 \mathrm{CH}$ $1 \div 5.39 .1 \mathrm{ICBC}$ $-101.036$ -1010131 $-101.428$ $-101.184$ $-101.120$ $-101.120$ $-101.284$ $-101.430$ $-101.394$ $-101.155$ $-101.266$ $-101.24 .7$ $-101.387$ $-101.149$ $-101.247$

$-101.311$

$-101.320$

$-101.281$

$-101.184$

$-101.362$

$-101.332$

$-100.886$

$-100.925$

$-100.908$

$-100.910$

$-100.996$

$-100.950$

$-100.931$

$-100.966$

$-100.540$

$-100.729$

$-100.419$

$-101.552$

-101.577
-101.679

$-101.6,5 ?$

$-101.700$

$-131.844$

$-101.779$ $-101.878$ $-101.933$ $-101.960$ $-191.47 A$ $-101.479$ $-101.557$

- (1) 1.493

$-101.256$
ACTUAL

TEMP

DEGREES C

37.6551

7.6736

37.6891

37.5737

38.1198

38.1339

39.0839

38.0619

39.0311

38.0819

$38.016 \mathrm{~A}$

$3 B .039 A$

8.0200

37.3605

37.9463

37.9678

37.

(1)

37.8648

37.9922
37.4810

37.8810
37.8719

37.9558

37.

37.7445

37.7714

37.7605

38.4741

38.4527

A.4109

$38.399 \mathrm{~A}$

38.3671

4.3722

38.352

38.3433

38.31343

ง..

37.69710

3.6no

$37.7 n d n$

9.6713

37.6677

37.6937

31.7115

3r.

?.6?

97.7194

$3 \mathrm{H} .4079$

ธ8. 3951

$3 R .36 .7$

3 c..5051

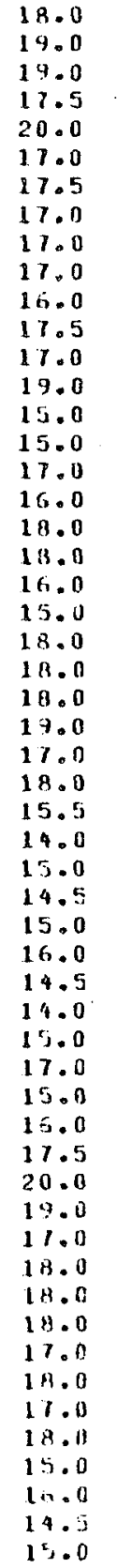

GEOTHERMUMCTER TEMPERATURES - DEGREES C

DUARTZ CHALCEOONY.

$N A-K$

NA-K-CA NA-K-CA 10

\begin{tabular}{|c|c|c|}
\hline $5 \varsigma .8$ & 25.4 & 242.0 \\
\hline 57.7 & 23.2 & 192.1 \\
\hline 62.1 & 29.3 & 211.7 \\
\hline 63.7 & 29.5 & 227.9 \\
\hline 55.0 & 25.4 & 226.9 \\
\hline 61.3 & 33.3 & 369.5 \\
\hline 63.7 & 29.5 & 210.2 \\
\hline 61.8 & 21.5 & 221.5 \\
\hline 61.3 & 33.5 & 245.5 \\
\hline 73.9 & 10.1 & 179.1 \\
\hline 55.8 & 25.4 & 235.8 \\
\hline $6 \div .7$ & 29.5 & 237.3 \\
\hline 62.7 & 29.5 & $198 . ?$ \\
\hline 59.8 & 25.1 & 252.8 \\
\hline 62.1 & 29.5 & 77.4 \\
\hline 59.9 & 25.4 & 77.9 \\
\hline 63.1 & 29.5 & 270.7 \\
\hline 65.5 & 31.4 & 131.4 \\
\hline 53.2 & 18.6 & 276.6 \\
\hline $5 \mathrm{C} \cdot \mathrm{A}$ & 16.1 & 227.9 \\
\hline 4.9 .3 & 13.5 & 63.1 \\
\hline 49.3 & 13.5 & 66.8 \\
\hline 4.8 .3 & 13.5 & 81.3 \\
\hline 48.3 & 13.5 & 222.3 \\
\hline 51.7 & 23.2 & 368.0 \\
\hline 65.5 & 31.4 & 293.3 \\
\hline 65.5 & 31.9 & 209.9 \\
\hline 63.7 & 29.5 & 265.5 \\
\hline 109.8 & 78.8 & $252 . A$ \\
\hline $10 E .9$ & 77.9 & 229.1 \\
\hline 99.1 & 0.101 & 129.3 \\
\hline 102.7 & 12.2 & 158.0 \\
\hline 83.5 & 56.9 & 244.8 \\
\hline 96.1 & 13.9 & 26509 \\
\hline 75.6 & 16.2 & 313.1 \\
\hline 80.2 & 17.1 & 256.3 \\
\hline 90.7 & 58.1 & 313.1 \\
\hline B:. 4 & 55.7 & 308.6 \\
\hline 89.6 & 56.3 & 236.9 \\
\hline 91.4 & 59.3 & 227.4 \\
\hline 75.6 & 45.2 & 232.2 \\
\hline 75.3 & 91.7 & 207.0 \\
\hline 61.8 & 27.5 & 199.1 \\
\hline 5.9 .8 & 25.4 & 221.11 \\
\hline 55.5 & 21.11 & 248.0 \\
\hline $5: 5$ & $21 . n$ & 226.2 \\
\hline $5 \% .11$ & 25.7 & 218.6 \\
\hline 49.3 & 13.5 & 229.1 \\
\hline $4 i: .3$ & 13.5 & 226.2 \\
\hline $4: .15$ & 10.7 & 230.0 \\
\hline $4 \leq 06$ & 10.1 & 237.5 \\
\hline 101.0 & $59 . ?$ & 222.9 \\
\hline 101.9 & 100.2 & 235.0 \\
\hline $10 \% .4$ & 71.2 & 240.0 \\
\hline $9 \div 1$ & 6.3 .7 & $2 B 8.7$ \\
\hline
\end{tabular}


WELL LOCATION

\begin{tabular}{|c|c|c|}
\hline NSHIF-RANGE & LONGI TUDE & LAII \\
\hline 19:3月ล3 EBA11 & -101.988 & 38.35 .42 \\
\hline $26531123(\mathrm{AD})$ & -100.679 & 37.7751 \\
\hline $26.314 \geq 1 C D C$ & $-100 \cdot 756$ & 37.7429 \\
\hline ?SS31 A3ECAC & -100.663 & 37.7455 \\
\hline $2 \leq \$ 32422404$ & -100.805 & 37.7955 \\
\hline $260.32135 \mathrm{ELA}$ & -100.790 & 37.7449 \\
\hline 2653.962 CAB!! & -100.896 & 97.7711 \\
\hline 2653942411014 & -100.991 & 37.7819 \\
\hline $27535417 \mathrm{ACO}$ & -101.16 .1 & 37.7057 \\
\hline 23536415000 & -101.234 & 37.6945 \\
\hline $27536 \mathrm{H} 1 \mathrm{ADCCH}$ & -101.295 & 37.7001 \\
\hline $21535 \times 260 \mathrm{CC}$ & -101.218 & 37.6591 \\
\hline $21537 \mathrm{~N}$ AAHB & -101.349 & 37.7402 \\
\hline $27.331: 11 \mathrm{AHA}$ & -101.330 & 37.7256 \\
\hline 285374220834 & -101.360 & 37.696 .2 \\
\hline $27358429 \mathrm{CHH}$ & -101.396 & 37.6741 \\
\hline $275354+12 A 0 C$ & -101.419 & $37.719 \mathrm{~A}$ \\
\hline $27538 \mathrm{~A} 22 \mathrm{CCA}$ & -101.467 & 37.6849 \\
\hline $21334+25 B$ UA & -101.433 & 37.65113 \\
\hline 27539429сгв & -101.506 & 37.6701 \\
\hline $2 \varepsilon S 351 \leq \mathrm{CHH}$ & -101.141 & 37.6156 \\
\hline $24335: 36 A U C$ & -101.096 & 37.5764 \\
\hline $20536420 \mathrm{COU}$ & -101.224 & 37.5397 \\
\hline $2.53141 \mathrm{CDCO}$ & -101.357 & 37.6321 \\
\hline $2053 \operatorname{lin} 3 \cos 00$ & -101.413 & 37.5916 \\
\hline ?85334 enf1H & -101.506 & 37.6372 \\
\hline $295301 \div 120 \mathrm{Co}$ & -101.433 & 37.6337 \\
\hline $2 F 538 W=10 \mathrm{An}$ & $-11) 1.515$ & 37.5714 \\
\hline $25335 \mathrm{~d} / \mathrm{CC}$ & -101.105 & 37.5511 \\
\hline 259352 EOAA & -101.188 & 37.5644 \\
\hline $25537 !$ 9CBA & -101.394 & 37.5122 \\
\hline 25.351 d25HHO & -1111.394 & 31.5037 \\
\hline $25539 \mathrm{HaCCEC}$ & -101.501 & 27.5014 \\
\hline $255331=2 \mathrm{ZHH}$ & -10.1 .470 & 37.5202 \\
\hline $30055 \mathrm{~A} 20 \mathrm{HC}$ & -101.114 & 37.4666 \\
\hline $3633511918 \mathrm{Cl}$ & $-101.19 ?$ & 37.1265 \\
\hline $30319430 C C$ & -101.460 & 37.4534 \\
\hline $3133941 \mathrm{xcr}$ & -101.433 & 37.4341 \\
\hline $3139384150 \mathrm{DPC}$ & -101.460 & 37.4378 \\
\hline 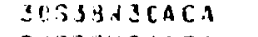 & -101.513 & 37.4111 \\
\hline 2 BS35U2EACA & -101.108 & $37.5 \mathrm{~Pa} 7$ \\
\hline 2ES314 2HA!1 & -101.341 & 37.6523 \\
\hline $2453412 \mathrm{coch}$ & -101.997 & 37.5970 \\
\hline $25.39423 \mathrm{AAA}$ & -101.463 & 37.5541 \\
\hline 300.3746000 & -101.406 & 31.4533 \\
\hline 3OSS9L CCA11 & -101.451 & $31.46,89$ \\
\hline 24.28127000 & -100.262 & 37.1550 \\
\hline ?CSPHAISABO & -100.421 & 37.1723 \\
\hline 253304?4000 & -100.5 .44 & 37.1112 \\
\hline $2630 \mathrm{~V} 280 \triangle A$ & -100.598 & 37.1619 \\
\hline 27327 eBA11S & $-100 \cdot 32.3$ & 37.8391 \\
\hline $27: 27,110 \mathrm{c}$ cII & -100.265 & 31.1135 \\
\hline $21823 \mathrm{in} \geq 41360$ & -100.339 & 37.6 .931 \\
\hline $21399 \sqrt{2} 3400$ & -100.455 & 57.5704 \\
\hline $210.294: 3000$ & -100.606 & .87 .6535 \\
\hline
\end{tabular}

ACTIUAL

TEMP

DEGREES C

15.

18.0

16.5
17.0

17.0

16.0

1 I. 5

17.5

18.0

19.0
18.0

18.0
17.5

19.0

17.5

17.5

17.0

16.5

18.0

17.5

20.0

19.0

19.0

19.0
18.0

18.0
18.0

19.0

11.5

19.0

19.0

18.0

19.0

18.0

18.0

19.0

17.0

19.0
19.0

19.0
19.0

18.0

19.11

19.0

20.0

18.0

$1 \mathrm{~B} . \mathrm{O}$

2.0

13.0

15.0

17.11

17.0

16.0

11.0

15.0

GE OTHETMOMETER TEMPFRATURES - DEGREES C

OUARTL CHALCEDONY NA-K NA-K-CA NA-K-CA LO

$$
\begin{aligned}
& \begin{array}{r}
102.8 \\
65.5 \\
70.7
\end{array} \\
& 70.7 \\
& 61.9 \\
& 6 \% .3 \\
& 61.3 \\
& 63.0 \\
& 53.2 \\
& 67.3 \\
& \begin{array}{l}
67.3 \\
57.7
\end{array} \\
& \text { 5). } 7 \\
& \text { औ1<. } 3 \\
& 8 ? .6 \\
& 78.2 \\
& 94.7 \\
& 76.8 \\
& 75.3 \\
& A<.3 \\
& 55.8 \\
& 63.7 \\
& 79.7 \\
& \text { B..6 } \\
& \begin{array}{l}
76.8 \\
82.3
\end{array} \\
& 82.3 \\
& 53.7 \\
& 65.5 \\
& 95.0 \\
& 63.0 \\
& 75.3 \\
& 67.3 \\
& 6.3 .1 \\
& 7 \mathrm{E} .8 \\
& 73.7 \\
& 51.2 \\
& \begin{array}{l}
51.8 \\
57.7
\end{array} \\
& 51.1 \\
& 5 \% .8 \\
& 79.6 \\
& 5 \pi .8 \\
& 69.0 \\
& 71.7 \\
& \text { fil. } 19 \\
& 69.0 \\
& 6 \div .7 \\
& 61.9 \\
& 7 \geq .8 \\
& 7 \mathrm{dj} .7 \\
& 7 \text { i). } 7 \\
& \begin{array}{l}
61.8 \\
65.5
\end{array}
\end{aligned}
$$

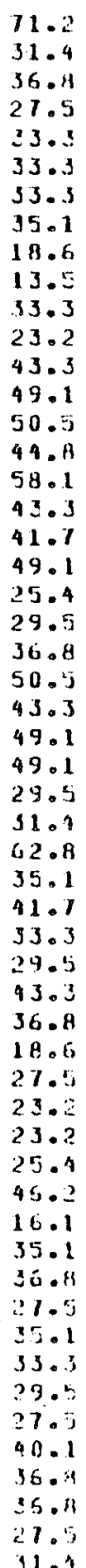

243.4

224.5

43.11

220.7

241.0

260.9

295.3

352.9

301.8

158.5

161.7
167.0

167.0
145.4

145.1

31.5

$30.2-1.5$

$2 \mathrm{H} .4-\mathrm{B} . \mathrm{S}$

23.7

20.1

11.4

46.5

46.5
44.2

44.2
58.2

5.5 .5

41.3

$175.2 \quad 41.0$

184.955 .9

203.3

175.2

208.9

208.9

233.7

233.1

153

153.4
183.6

158.3

139.0

205.8

193.9

130.1

213.6

209.7

213.1

211.5

218.5

191.4

$19 \% .3$

175.1

1H2. 1

200.6

225.1

116.9

159.0

200.1

197.5

200.9

258.1

254.0

265.0

256.9

292.4
294.2

284.2

273.3

41.6

39.0

30.6

30.6

43.3

43.3
47.6

47.6
46.9

43.0

46.0

35.7

$34 . \mathrm{A}$

49.2

42.0

46.3

39.5
41.5

48.7

4 .. 7

99.5
$3 \% .8$

$3 \% .8$
94.7

11.7

40.2

31.2

40.6

40.9

40.9

4.3 .9

4.3 .9

32.6

33.3

31.2

33.4

31.6

35.2

33.7

$-9.6$

$-12.2$

$-14.5$

-1.9
3.6

3.4

10.7

16.0

1.7

10.1

1. $\mathrm{H}$

-1
-.1

$-1$

$-3.4$

$-3.4$

$-.3$

6. $A$

2. 1

3. 3

4.7
-.9

10.4

4.6

4.

4.0

4.0

4.3
5.3

10.8

2.7

6. 1

4.1

4.2
-6.2

$-. ?$

32.6

4.2

4.9
5.5
-3.2
-3.4

$-3.2$

-6.5
-7.5

-7.5
-2.9

$-4$.

$-2 \cdot k$

-5.7
-10.9 
WELL LOCATION

\begin{tabular}{|c|c|c|}
\hline DHIF-RANGE & LONGI TUDE & LATI TUOE \\
\hline $2152913 \in A H H$ & -100.492 & 37.5665 \\
\hline $21590 \mathrm{~W}$ PBOH:H & -100.1332 & 37.1252 \\
\hline 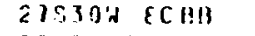 & -100.632 & 37.7178 \\
\hline $28530+22 A C C$ & -100.591 & 37.6903 \\
\hline $27530 \mathrm{ti} 25 \mathrm{CCH}$ & -100.560 & 31.6101 \\
\hline $275304340 \mathrm{CC}$ & -100.596 & 37.6536 \\
\hline 245212 4A (143 & -100.278 & 37.6509 \\
\hline 2ЯS2 HW2CACD & -100.399 & 37.6019 \\
\hline 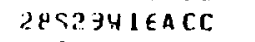 & -100.497 & 37.6168 \\
\hline 2PCBOA EHHA & -100.648 & 37.6517 \\
\hline 28530\%1CODO & -100.580 & 37.6242 \\
\hline $2 H 530 \times 17 \mathrm{HHA}$ & $-100.6,30$ & 31.622 .3 \\
\hline $28.3304290 \mathrm{All}$ & -100.556 & 37.5077 \\
\hline$=0530 \mathrm{WJ1C \textrm {CH }}$ & -100.650 & 37.5672 \\
\hline $25.527 \times 30 \mathrm{HCC}$ & -100.323 & 37.4283 \\
\hline$\therefore \Xi S \supseteq R H \unlhd \in A B A$ & -100.222 & $37.4 B R O$ \\
\hline $2 \div 52$ did $1 \mathrm{ACCC}$ & -100.133 & 31.5212 \\
\hline $23623+13000$ & -100.417 & 37.5064 \\
\hline $2 \operatorname{ss} 28 \omega 2 \theta c 00$ & -100.392 & 37.4915 \\
\hline 2. 35294105134 & -100.488 & 37.5488 \\
\hline 253294271100 & -100.488 & 37.5010 \\
\hline $23 S 30 \mathrm{~W} 2 \mathrm{HBHC}$ & -3.00 .596 & 37.5176 \\
\hline $25330435 \mathrm{CC}$ & $-100.5,67$ & 37.41145 \\
\hline $2 r .54 .1 \mathrm{~W} 2 \mathrm{cos} n \mathrm{O}$ & -101.831 & 37.7815 \\
\hline $2 E S 42122 C D U$ & -101.901 & 37.7125 \\
\hline $26843+250 \mathrm{CC}$ & -101.969 & 37.7560 \\
\hline $27 S 31 W \angle 1 H 40$ & -100.123 & 37.6947 \\
\hline $27831924 \mathrm{coc}$ & -100.564 & 37.6 .930 \\
\hline $2.331 \mathrm{~W} 31 \mathrm{CHC}$ & -100.760 & 37.6583 \\
\hline $27332 \mathrm{~N} \in \mathrm{CHB}$ & -100.869 & 37.1313 \\
\hline 21532 H1 2 A HII & -100.769 & 37.1117 \\
\hline $27532 W 13 C \mathrm{CD}$ & -100.866 & 37.6845 \\
\hline $275331270 \mathrm{nO}$ & -100.916 & 37.6735 \\
\hline $27533: 250 A A$ & -100.944 & 37.6753 \\
\hline $27833 \mathrm{H330 \textrm {CO }}$ & -100.930 & 37.6551 \\
\hline 21534415000 & -101.035 & 37.6786 \\
\hline $21 S 34 \mathrm{HEQOAA}$ & -101.935 & 37.6797 \\
\hline 29931:32A Un & -100.732 & 37.57 9 \\
\hline $20531+35 \cos$ & -100.687 & 37.5675 \\
\hline $2953219 \mathrm{GCC}$ & -100.832 & 37.6244 \\
\hline 28532418884 & -100.8699 & 37.6237 \\
\hline $245321435 H 1 A A$ & -100.794 & 37.5792 \\
\hline $249.53 \mathrm{H} 1 \mathrm{HCC}$ & -100.741 & 37.6035 \\
\hline 29533424060 & -100.818 & 37.5908 \\
\hline $289344150 \mathrm{Als}$ & -101.619 & 37.6160 \\
\hline$=9534432 A C D$ & -101.053 & 31.5734 \\
\hline 25851614091111 & $-1 \cup C . G R T$ & 31.5344 \\
\hline $25531 W: 4 B C A$ & -100.103 & $37.4 \pi 67$ \\
\hline $275324 \quad 4300$ & -100.832 & 37.5591 \\
\hline $2033 \% \$ 160$ AA & -100.015 & 37.5277 \\
\hline 250331 1AAII & -100.973 & 31.5548 \\
\hline $2 \div 533 U$ EACA. & $-1010.94 P$ & 31.5512 \\
\hline 20033АС АНСО & -100.341 & $31.592 ?$ \\
\hline $2953+42 A+13$ & -101.005 & 51.5646 \\
\hline 283554114010 & -100.405 & 57.54112 \\
\hline
\end{tabular}

ACTUAL

DEGREES C

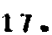

17.0
16.0

16.0

16.0

16.0

16.0

11.0

17.0

17.0

15.0
16.5

16.0

17.0

17.0

17.0

16.5

16.0

16.0

16.0

16.0

16.00

15.0

16.5

17.5
18.0

18.0
16.0

16.0

24.0

17.0

$17 . n$

18.5

17.0

17.5

17.5

$2 . .0$

18.0
19.0

19.0
18.0

18.0

18.5

17.5

17.5

11.5

19.0

19.0

190

22.5

19.0

17.5

17.5

19.0

19.0

13.

19.0

14.0

19.0
GLO OHEKMOMETER TEMPERAIURE.S - DEGREES C

OUARI/ CHALCEUONY NA-K NA-K-CA NA-K-CALO

63.729.

67.3

$75 \cdot 3$

70.7

E.

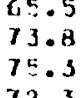

75.3

65.5

69.0

61.3

65.0

65.0

84.8

9.8.6

70.7

73.

73.6

69.0
72.3

72.3
70.7

69.0

$7 E .8$

55.5

53.2

55.8

61.3

67.3

65.05

6 ...

63.0
67.3

55.5

65.5
65.5
6.53

61.3

$6 \geq .7$

61.3

65.0

69.0

63.0

76.7

63.7

$6: .3$

$6: .3$
6.3 .1

6.5 .1
$7 \leq .3$

69.0

ii).

$7 ? .3$

70.1

61.

61.9

$6: .7$
280.5

208.0

223.5

276.6

256.9

252.8

268.0

317.5

260.1

254.6

258.

24 A.

$26,1.5$

303.5

317.5

$40 B=5$

311.1

298.5

304.5

276.6

291.6

251.7

216.6

1Bf.

269.9

254.

244.1

267.5

316.3

233.2

47.5

271.1
285.5

245.5
259.8

259.8
201.7

242.5

235.

276.6

299.5

266.2

242.0

199.2

238.1

81. 8

204.7

231.4

266.2

229.4

183.9

175.2

$21 \% .3$

220.8

200.1

209.1

\begin{tabular}{|c|c|}
\hline 31.1 & -7.5 \\
\hline 31.1 & -1.1 \\
\hline $\begin{array}{l}27.5 \\
32.3\end{array}$ & $\begin{array}{l}-8.1 \\
-4.6\end{array}$ \\
\hline $\begin{array}{l}32.3 \\
21.5\end{array}$ & -10.3 \\
\hline 21.2 & $-10 . H$ \\
\hline 30.3 & -4.9 \\
\hline 34.5 & -2.4 \\
\hline 29.0 & -7.9 \\
\hline 26.1 & -8.0 \\
\hline 25.7 & $-B .1$ \\
\hline 21.6 & -3.7 \\
\hline $27 . A$ & -1.7 \\
\hline 22.2 & -10.6 \\
\hline 32.5 & $-4 \cdot 8$ \\
\hline 28.9 & -7.7 \\
\hline 32.0 & -5.4 \\
\hline 30.7 & -4.1 \\
\hline 31.4 & $-4 \cdot 9$ \\
\hline $2 \theta .4$ & -7.3 \\
\hline 28.7 & -7.4 \\
\hline 26.5 & -5.1 \\
\hline 26.1 & -7.8 \\
\hline 47.4 & 2.9 \\
\hline 34.9 & -3.0 \\
\hline 36.0 & -1.2 \\
\hline 25.8 & -11.0 \\
\hline 25.4 & -9.4 \\
\hline$? 1.5$ & -10.0 \\
\hline 25.3 & -11.2 \\
\hline 27.8 & -9.8 \\
\hline 22.0 & -11.2 \\
\hline 32.7 & 3.0 \\
\hline 22.5 & -10.4 \\
\hline 29.0 & -9.3 \\
\hline 24.3 & -10.9 \\
\hline 25.7 & -6.9 \\
\hline 23.5 & -9.6 \\
\hline 23.9 & -9.4 \\
\hline 22.6 & -11.1 \\
\hline 24.9 & -9.8 \\
\hline 21.5 & -4.2 \\
\hline 30.1 & -3.6 \\
\hline 12.9 & 6.2 \\
\hline 33.1 & -1.9 \\
\hline 31.2 & -3.0 \\
\hline 25.1 & -9.6 \\
\hline 29.1 & -5.4 \\
\hline 32.4 & -2.1 \\
\hline 35.1 &.$- B$ \\
\hline 29.9 & $-4 \cdot 11$ \\
\hline 39.8 & -.1 \\
\hline 35.0 & -.2 \\
\hline 32.7 & -.6 \\
\hline 32.0 & -4.1 \\
\hline
\end{tabular}


HELL LOCATION

\begin{tabular}{|c|c|c|}
\hline WNSHIP-RANGE & LONGITUOE & \\
\hline 20534190011 & -101.1151 & 31.542 \\
\hline 23339111400 & -100.998 & 37.5445 \\
\hline $2993422411 \mathrm{CC}$ & -100.796 & 37.5150 \\
\hline $25.53923 \mathrm{ECAC}$ & -100.996 & 37.4819 \\
\hline $90531 \% 68318$ & -100.760 & 37.4760 \\
\hline 3OS31W1SAHB & -100.596 & 37.4952 \\
\hline $20531424 B H B$ & -100.669 & 37.4314 \\
\hline 0053113301804 & -100.723 & 37.4022 \\
\hline xes32411!13月 & -100.196 & 37.4614 \\
\hline $30532417 \mathrm{BCC}$ & -100.850 & 37.442 \\
\hline $3035243118 \mathrm{AB}$ & -100.1364 & 37.402 \\
\hline $\operatorname{ses} 3342$ AnAll & $-10 \mathrm{c} . \mathrm{A91}$ & 37.485 \\
\hline 305334 e0HA & -100.966 & 37.467 \\
\hline $3 \operatorname{css} 3430 \mathrm{CHO}$ & -100.975 & 37.4089 \\
\hline 305342 EHHH & -101.069 & 37.4759 \\
\hline $\operatorname{son} 3411$ ó & -101.051 & 37.9766 \\
\hline 26:335.310 A & -101.184 & 37.7145 \\
\hline $2635: 3 d 160013$ & -101.254 & $37.7 \mathrm{AB}$ \\
\hline $2 E 331122 \mathrm{CCA}$ & -101.357 & 37.773 \\
\hline 25.53626018 & -101.332 & $31.7(6.0$ \\
\hline 3052644 CHA & -100.179 & 37.465 \\
\hline $305254 \quad 76411$ & -100.216 & 31.458 \\
\hline $\operatorname{SCS26HIZBBC}$ & -100.197 & 37.441 \\
\hline $30525 \mathrm{~s} 3 \mathrm{ICHC}$ & -100.216 & 37.3202 \\
\hline $305274 \quad 40 E 0$ & -100.276 & $37.4 \mathrm{Ka}$ \\
\hline $20 \div 28123 A H 11$ & -100.243 & 37.423 \\
\hline $30526 W 2 B A A$ & -100.353 & 37.474 \\
\hline $30929 \times 17 A B 11$ & -100.106 & 37.945 \\
\hline $30.52 \% 300 C \mathrm{C}$ & -100.479 & 37.46 .2 \\
\hline $3 \because 52942 \geq 0 A 0$ & -100.463 & 37.4220 \\
\hline 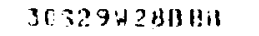 & -100.506 & 37.415 \\
\hline $\cos 50$ a $12 \mathrm{CHII}$ & -100.560 & 37.453 \\
\hline $\cos 30 \% 2 H A H H$ & -100.606 & 37.416 \\
\hline$\therefore 1.28 \mathrm{~A} 1 \mathrm{OHCH}$ & -100.362 & 37.364 \\
\hline IIS?UVIEAAA & -100.416 & 37.32 \\
\hline$\because 1529 W \Omega C A A \Lambda$ & -100.508 & 37.325 \\
\hline $31830142 \in B H C$ & $-100 \cdot 597$ & 37.358 \\
\hline 2CS3OW SCCC & -100.597 & 37.21 \\
\hline $22530.2239 B C$ & -100.596 & 37.240 \\
\hline $2033043 E C A A$ & -100.553 & 37.221 \\
\hline $3: C ?, Y H \geq \in A A U$ & -160.019 & 37.141 \\
\hline $31553 \times 190 \mathrm{acc}$ & -101.609 & 37.341 \\
\hline$\because 15904 \geqslant 9 A B 14$ & -101.690 & 37.328 \\
\hline$? 1002225 n$ All & -131.703 & 37.325 \\
\hline$\therefore \sin 3: 11 / 000$ & -102.022 & 31.345 \\
\hline$? 2740: 1 ? 1 \mathrm{AOBU}$ & -101.5657 & 37.25 \\
\hline $32542 i+14 C C C$ & $-101 . \$ 152$ & 37.258 \\
\hline$\therefore \triangle S 421 \mathrm{~N} \geq 1 \mathrm{HCC}$ & -101.1198 & $3 r .2 r_{i}$ \\
\hline $3 \geq 5,2428 \mathrm{COn}$ & -1 in1 . 855 & 31.227 \\
\hline 92343611000 & -102.017 & $37 . ? 5$ \\
\hline 马.5.5OW16A1113 & -101.562 & 37.18 \\
\hline$\therefore 340$ 27ccc & -101.061 & 37.14 \\
\hline $39541: 3440$ & -1111.155 & $3 r .21$ \\
\hline$\therefore 500 \mathrm{~W} 241311$ & -101.661 & 37.03 \\
\hline 25434124014 & & $37 \cdot 1$ \\
\hline
\end{tabular}

ACTUAL

IEMP

DEGREES C

\begin{tabular}{|c|c|c|}
\hline 19.0 & & 61.3 \\
\hline 19.0 & 1 & $6 E .5$ \\
\hline $\begin{array}{l}19.0 \\
19.0\end{array}$ & & $6 \div 5$ \\
\hline 18.0 & & 65.5 \\
\hline 17.5 & & 65.5 \\
\hline 17.5 & & 63.7 \\
\hline 18.0 & & 65.5 \\
\hline 18.0 & & 65.5 \\
\hline 18.0 & & 59.8 \\
\hline 18.0 & & 65.5 \\
\hline 19.0 & & 63.7 \\
\hline 19.0 & & 61.3 \\
\hline $21 . \overline{7}$ & & 65.0 \\
\hline 19.5 & & 67.3 \\
\hline 19.5 & & 70.7 \\
\hline 18.0 & & 65.5 \\
\hline 17.0 & & 55.5 \\
\hline 17.0 & & $6,5.5$ \\
\hline 18.0 & & 67.3 \\
\hline 15.0 & & 75.3 \\
\hline 15.0 & & 70.7 \\
\hline 15.0 & & 61.3 \\
\hline 18.0 & & 82.3 \\
\hline 15.0 & & 67.3 \\
\hline 16.0 & & 61.3 \\
\hline 16.0 & & 70.1 \\
\hline 15.5 & & 57.7 \\
\hline 16.5 & & 67.3 \\
\hline 16.0 & & 51.7 \\
\hline 11.0 & & 61.8 \\
\hline 18.0 & & 51.1 \\
\hline 14.0 & & $6=.5$ \\
\hline 16.5 & & 53. . \\
\hline 16.0 & & 55.8 \\
\hline 16,01$)$ & & 73.8 \\
\hline 14.0 & & 63.0 \\
\hline 1 A. 0 & & 61.3 \\
\hline 16.0 & & 79.9 \\
\hline 17.0 & & 73.6 \\
\hline lis.o 0 & & 75.3 \\
\hline 18.0 & & 73.3 \\
\hline 18.0 & & 72.3 \\
\hline 21.0 & & 55.5 \\
\hline 18.0 & & $5 \div .4$ \\
\hline 19.0 & & 83.5 \\
\hline 18.0 & & 76.8 \\
\hline $1 R .0$ & & 76.8 \\
\hline 19.3 & & 76.3 \\
\hline 19.0 & & 82.3 \\
\hline 19.0 & & S.'S \\
\hline 19.0 & & 79.6 \\
\hline $1 \% .0$ & & 81.6 \\
\hline 23.0 & & 79.6 \\
\hline 19.0 & & 72.3 \\
\hline
\end{tabular}

GË OTHERMOMETER TEMPERATURES - DEgRLES C

QUARTE CHALCEUONY NA-K NA-K-CA NA-K-CA LO

$61.3 \quad 27.5$

31.4

201.0

198.4

172.8

190.

226.1

259.1

256.9

232.

230.5

221.7

196.6

205.9

190.3

187.5

185.4

180.9

298.9

324.

324.0

260.

282.

299.

273.

276.6

240.6

280 .

268.5

308.

318.1

305.

281.7

259.1

250.0

250.0

237.8

310.4

281.9

276.6

255.0

235.8

225.7

222 .

42.0

198.2

203.2

124.5

196.

172.

172 .

119.

183.7
213.7

168 .

121.9

$177 . ?$

137.9

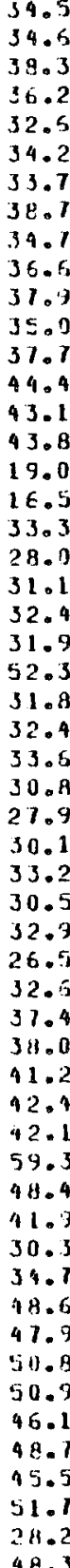

.6

.6

4

$-2.5$

$-.6$

1.9

1.9

$-1.0$

- 2

.1

3.2

6.7

i. 2

6.6

$-14.2$

$-5.1$

$-5.1$

$-4.8$

$-6 \cdot 3$

$-5.1$

10.3

$-4.0$

$-4.1$

$-2.4$

$-3.2$

$-5.2$

$-5.5$

$-2.2$

$-3.2$

$-2.4$

$-2.4$

$-3.8$

$-.2$

2. 5

3.8

4.1

4.4

15.6

6.4

6.0
-6.9

-6.5
-.7

5.8

10.4

11.3

3.4

1.6

7.0 
HELL LOCATION

\begin{tabular}{|c|c|c|}
\hline IOXNSHIP-PANGE & IONGI TUOE & LAT \\
\hline :15314 AHCC & -100.722 & 37.3577 \\
\hline $31531 \mathrm{NLACH}$ & -100.652 & 37.3547 \\
\hline IS.32N ICAD & -100.779 & 37.3787 \\
\hline $31532 \mathrm{~d} 21 \mathrm{CHA}$ & -100.910 & 37.3372 \\
\hline$=1532 \mathrm{~d}: 4 \mathrm{CH}$ & -100.794 & 37.3092 \\
\hline $3133.3 \mathrm{~N} 6 \mathrm{C}(13 !)$ & -100.956 & 37.3791 \\
\hline$\because 1533 n 2 \mathrm{COH}$ & -100.331 & 37.337 \\
\hline D1539WLABUH & -101.067 & 37.358 \\
\hline 229314 BOHH & -100.722 & $37.2 \mathrm{HF}$ \\
\hline ICS31:2ECAA & $-100 . f_{f}, 2 ?$ & 37.235 \\
\hline $22531231 \mathrm{ACC}$ & -100.731 & 37.2231 \\
\hline $32532 \times 14043$ & -100.716 & 37.2720 \\
\hline ICS32WIgUAOI & -160.8444 & 37.257 \\
\hline $3 \angle 533+1 \angle H C C$ & $-100,867$ & 37.281 \\
\hline .2539417000 & -101.581 & 37.253 \\
\hline 325341432000 & -101.748 & 37.229 \\
\hline $22333412 A A 0$ & - 100.0550 & $37.1 \%$ \\
\hline 3ACS?AZSADA & -100.759 & 37.051 \\
\hline $345344160 A A$ & -101.013 & 37.0903 \\
\hline Y.333W16U1CA & -100.917 & 37.007 \\
\hline 9553441CBOH & -101.011 & 37.025 \\
\hline 21,3$)$ in $23 \mathrm{ACC}$ & -101.552 & 31.6900 \\
\hline $2133+8 \div 7$ BAA & -101.577 & 37.6807 \\
\hline 213394340010 & -101.503 & 37.6534 \\
\hline 27841619040 & -101.844 & 37.6737 \\
\hline $27541 \mathrm{A1C \textrm {CH }}$ & -101.951 & 37.6552 \\
\hline 27542. 310 CC & -101.960 & 37.6233 \\
\hline $21329 \mathrm{~d} 90 \mathrm{~A}$ & -100.491 & 37.7170 \\
\hline 2054143600 & -101.159 & 27.7919 \\
\hline $25-3149 \mathrm{CH}$ & -100.722 & 37.5411 \\
\hline $23532: 190 C$ & -100.1867 & 37.5385 \\
\hline I $\angle S 2 J 4$ ECC & -100.504 & 37.2805 \\
\hline 3.57842940 & -100.395 & 37.150 \\
\hline $23030 \mathrm{stc} 011$ & -100.558 & 37.1328 \\
\hline$\because 1540$ I 10A & -101.613 & 37.3798 \\
\hline $31543 \mathrm{~N} 3 \mathrm{CH}$ & -101.9139 & 37.3194 \\
\hline 325394 SDC & -161.557 & 87.2743 \\
\hline $3253421(1) A$ & -130.997 & 37.218 \\
\hline $27343 \% 120 \mathrm{CC}$ & -101.778 & 36.1194 \\
\hline EITAJ4 200 AH & -102.038 & 37.6736 \\
\hline 213591513013 & $-101,6,16$ & 37.651 \\
\hline $2: 20394.31300$ & $-101.6,34$ & 37.5125 \\
\hline 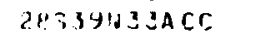 & -101.538 & 37.572 \\
\hline 2HOJJUSRABH & -101.533 & 37.579 \\
\hline $2994064 C C C$ & -1 iv 1.100 & $97.0: 9$ \\
\hline 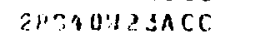 & $-101.6,61$ & 37.501 \\
\hline $203404=20 \mathrm{CO}$ & -101.724 & $37.5 i 31$ \\
\hline $26: 414 \div 11100$ & -131.6445 & .57 .5128 \\
\hline $2 \mu \mathrm{at}+\mathrm{acc}$ & -101.942 & $18.6 ? 40$ \\
\hline 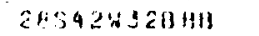 & -101.942 & 37.518 \\
\hline 25059u1 7ich & -101.116 & 37.530 \\
\hline $353334=1000$ & -101.366 & $3 \pi .510$ \\
\hline $2(59) 1=4004$ & $-131.52 \%$ & נ98 \\
\hline $25.921413 A C C$ & $-101 \cdot 152$ & 31.528 \\
\hline ?95414:1Ca11 & -101.849 & $11.4 \mathrm{~nL}$ \\
\hline
\end{tabular}

ACTUAL

DEGREES C

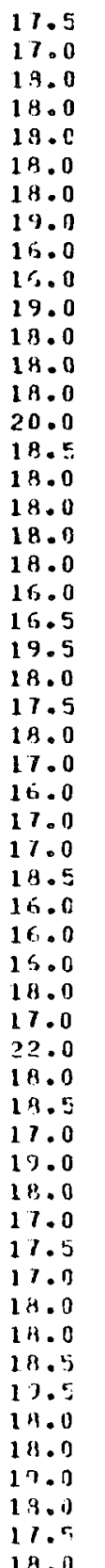

GE OTHERHOMETER TEMPER ATURES - DEGREES C

GUARIZ CHALCEDONY NA-K NA-K-CA NA-K-CA LO

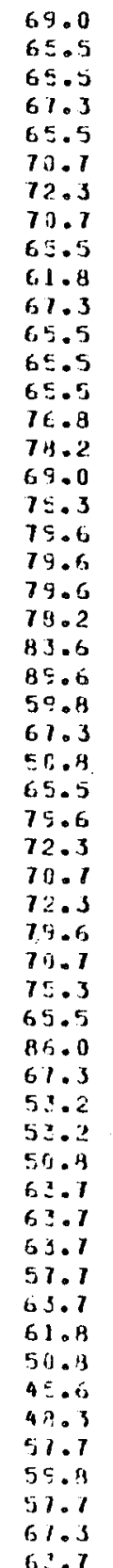

\section{6}

$228 \cdot 3$

189.1

189.7

189.

202.

196.

191.2

215.9

226 .

187.5

201.1

227 .

245 .

204.3

194.7

215.9

215.9

88.
228 .

228.9

220 .

150.5

244.

240.0

192.6

243.8

251.1

226.9

266.9

198.8

251.7

201.9

269.2

269.2

104.3

231.5

202.0

170.5

163.8
226.9

226.9
250.0

255.0

138.9

229.6

216.9

224.

224.3

235.1

235.1

216.

351.9

$21 \mathrm{~B} .4$
$1 \mathrm{A3} .3$

234.0

212.1

221.1

21109

\begin{tabular}{|c|c|}
\hline $\begin{array}{l}41.5 \\
38.4\end{array}$ & $\begin{array}{l}5.1 \\
2.9\end{array}$ \\
\hline $\begin{array}{l}38.4 \\
38.3\end{array}$ & $\begin{array}{r}2.9 \\
.5\end{array}$ \\
\hline 94.2 & 5.5 \\
\hline 44.6 & B. 2 \\
\hline 43.4 & $\therefore, 2$ \\
\hline 43.9 & 10.9 \\
\hline 55.1 & 12.9 \\
\hline 40.4 & 4.1 \\
\hline 42.1 & 5.5 \\
\hline 45.8 & 9.1 \\
\hline 15.1 & 5.0 \\
\hline 49.8 & 8.5 \\
\hline 51.1 & 12.0 \\
\hline 41.3 & 3.1 \\
\hline 46.1 & 6.1 \\
\hline 36.9 & .7 \\
\hline 51.2 & 13.0 \\
\hline 24.2 & -6.3 \\
\hline 36.3 & 2.6 \\
\hline $3 \Xi .7$ & -.6 \\
\hline 39.5 & -4.7 \\
\hline 3 ค. 0 & 1.3 \\
\hline 39.5 & 2.7 \\
\hline 37.2 & 1.1 \\
\hline 37.3 & 2.5 \\
\hline 34.6 & -.5 \\
\hline 36.1 & -5.4 \\
\hline 45.2 & 2.6 \\
\hline 25.3 & -10.3 \\
\hline 35.7 & .6 \\
\hline 36.5 & -.9 \\
\hline $4 f_{0} .9$ & 6.5 \\
\hline 42.9 & 4.9 \\
\hline 45.9 & A. 5 \\
\hline $4 \times .3$ & 9.1 \\
\hline 53.4 & 12.7 \\
\hline 47.6 & 10.6 \\
\hline 35.5 & -1.1 \\
\hline 33.9 & -1.2 \\
\hline 52.3 & 12.1 \\
\hline 37.4 & 3.7 \\
\hline 39.3 & 4.1 \\
\hline$\therefore 9.5$ & 3.2 \\
\hline 36.0 & -01 \\
\hline 31.7 & .7 \\
\hline 37.5 & 2.1 \\
\hline 36.2 & -.3 \\
\hline 34.81 & $-2 \cdot B$ \\
\hline 49.0 & 2.1 \\
\hline 51.3 & 5.0 \\
\hline 37.9 & 4.0 \\
\hline 37.6 & 1.3 \\
\hline 39.1 & 3.5 \\
\hline .55 .4 & .1 \\
\hline
\end{tabular}


WELL LOCATION

\begin{tabular}{|c|c|c|}
\hline SHLP-PANGE & I.UNGITUDE & L \\
\hline $2594248 C \cap C$ & $-102.93 A$ & 37.5360 \\
\hline $23942: 24000$ & -101.870 & 37.5068 \\
\hline $303330<0119$ & $-101.55 ?$ & 31.476 .2 \\
\hline ZCS3 3 W 1 ABHU & -101.6 .35 & 37.9454 \\
\hline $30.5334: 320 \mathrm{~A}$ & $-101 . t_{1} 01$ & 37.3945 \\
\hline $3083343 \in H C D$ & -101.536 & 37.3975 \\
\hline $315401240 \mathrm{CC}$ & -101.648 & 37.1190 \\
\hline $30540433 \mathrm{CCB}$ & -101.707 & 37.3917 \\
\hline $3054201<A C C$ & -101.861 & 37.4555 \\
\hline $\sin 4221601304$ & -101.920 & 37.1426 \\
\hline $30513: 1346418$ & -1.12 .017 & 31.4023 \\
\hline BSISIIEAAA & -1111.114 & 31.3587 \\
\hline$\therefore 15 S \mathrm{JU} 2 \mathrm{EOCC}$ & -101.094 & 37.3169 \\
\hline $31335420 \mathrm{co}$ & -101.204 & 37.3754 \\
\hline ISS3H1ABAA & -101.276 & 37.3592 \\
\hline $3133 \sin 2111 \mathrm{Cl}$ & -101.228 & 37.3264 \\
\hline 3153749606 & -191.355 & $37.3 \sin 3$ \\
\hline $.31331 \times 2211 \mathrm{CC}$ & -101.337 & 37.3392 \\
\hline 31539 N1CCA & -101.975 & 37.3436 \\
\hline $31.33912311 \mathrm{A13}$ & -101.536 & 37.3448 \\
\hline $32535420 \mathrm{ong}$ & -101.103 & 37.2733 \\
\hline $32: 351800 n$ & -101.140 & 37.2735 \\
\hline $34 \$ 36: 111000$ & -101.196 & 37.1865 \\
\hline $38537+170 \mathrm{cos}$ & -141.372 & 37.1819 \\
\hline ISSSG: GAABS & -101.996 & 37.2143 \\
\hline $23338+1 C A C C$ & -101.436 & 37.1940 \\
\hline $9 \sin 3 i+2$ CCCB & -101.468 & 37.1597 \\
\hline 3ะ538HZ5OCC & -101.395 & 37.1428 \\
\hline 31595 ' $711 \mathrm{cc}$ & -101.173 & 37.106 .5 \\
\hline S4535'N18BCA & -101.111 & 37.0937 \\
\hline sassind 2 cousts & -101.922 & 37.1157 \\
\hline $14534 \mathrm{WLCAD}$ & -101.546 & 37.0990 \\
\hline $353551 \mathrm{AnA}$ & -101.175 & $3 \% .0 \times 3$ \\
\hline $3: 333 d 100 A 1)$ & -101.545 & 37.0155 \\
\hline 3.543 .321160 & -142.026 & 37.1312 \\
\hline $20 \cos 14 \quad 3 A 113$ & -98.5 .26 & 34.3969 \\
\hline $3951141.5 \mathrm{~A}$ & -94.577 & 38.3005 \\
\hline 203114260014 & -98.503 & 38.2179 \\
\hline $2 ! 58 ? 4 \quad$ ic & $-98,6,195$ & 38.3368 \\
\hline $24312 \% 34$ & -18.642 & 34.3942 \\
\hline $26: 3124 \in A A C$ & -98.5387 & 38.3453 \\
\hline 205129901004 & -98.696 & 38.2458 \\
\hline $20.31 .545 \mathrm{CrH}$ & -48.810 & 34.2731 \\
\hline ?1513:914013 & -98.303 & 31.2596 \\
\hline$=1: 11111$ CAAA & -98.9 .49 & 39.3330 \\
\hline 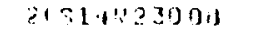 & -98.933 & 38.2731 \\
\hline 2031 1427CAC: & -48.56 .0 & 38.20104 \\
\hline 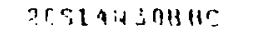 & -98.920 & 39.2877 \\
\hline $2 r, 81+32 H C A$ & -ун11. & $38.211 \%$ \\
\hline $24 S 1 / W 25 A A B$ & -99.133 & 37.9391 \\
\hline 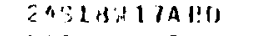 & -99.320 & $37.95 ? 1$ \\
\hline $24: 14596008$ & -94.744 & 37.3174 \\
\hline 369254 40148 & -49.085 & 37.71131 \\
\hline er & -99.011 & 37.8298 \\
\hline 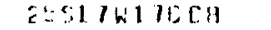 & .3 .209 & 37.91 \\
\hline
\end{tabular}

AC TUAL

TEIFF
DF GREES

\section{0}

17.0

18.0

17.0

17.5

18.0

17.5

11.5

19.0
17.0

17.0
17.0

19.0

18.5

19.0

19.0

19.0

18.13

18.0

19.0

18.0

18.0

$2 ? .0$

19.

19.0
19.0

19.0
19.0

19.0
19.0

19.0
19.0

20.0

20.1

20.0

2.1.

2.1 .0

20.0

13.

15.0

15.0
14.0

14.0
14.0

14.0
15.0

15.0
15.0

15.0

15.0

15.11

15

150

$1 \div, 0$

17.0

15.5
15.0

1500
15.0

14.0

$18 . n$

16.0
15.0
Ge OIHERMOMETER TEMPERATURES - DEGHEES C

QUARTZ CHALCEDONY NA-K

\section{7}

201.7 .91 .5

$207.0 \quad 43.8 \quad 1.7$

$211.3 \quad 39.2 \quad 4.2$

$\begin{array}{lll}197.1 & 40.9 & 3.9 \\ 203.9 & 49.3 & 9.2\end{array}$

$185.2 \quad 53.7 \quad 11.9$

$\begin{array}{rrr}190.1 & 99.0 & 9.4 \\ 210.8 & 51.5 & 12.7\end{array}$

$\begin{array}{lll}210.4 & 91.5 & 12.8 \\ 192.4 & 49.4 & 8.5\end{array}$

$222.5 \quad 39.1 \quad 2.1$

$\begin{array}{lll}154.9 & 47.0 & 6.1 \\ 198.2 & 50.2 & 8.3\end{array}$

$226.4 \quad 99.8 \quad 11.0$

$200.6 \quad 52.1 \quad 11.2$

192.8 $41.1 \quad 9.0$

$192.8 \quad 47.1 \quad 7.0$

$175.6 \quad 55.5$

$191.1 \quad 50.8 \quad 10.8$

$193.250 .4 \quad 11.6$

$237.0 \quad 48.3 \quad 9.9$

$\begin{array}{lll}197.5 & 48.7 & 13.4 \\ 206.5 & 11.0 & 3.0\end{array}$

$173.6 \quad 34.0 \quad-2.4$

$165.0 \quad 44.8 \quad 5.5$

$178.9 \quad 45.9$

$191.3 \quad 29.1 \quad-4.4$

$213.8 \quad 27.7 \quad-6.7$

$\begin{array}{lll}267.5 & 21.2 & -10.1 \\ 253.3 & 21.2 & -13.9\end{array}$

$196 . A \quad 31.8 \quad-4.2$

$322.6 \quad 20.3 \quad-15.4$

$276.6 \quad 30.1$

$205.5 \quad 20.5 \quad-13.0$

$\begin{array}{lll}105.4 & 30.2 & 11.1 \\ 103.3 & 41.3 & -1.1\end{array}$

$68.3 \quad 49.8$

$41.3 \quad 58.1 \quad 14.0$

$109.8 \quad 11.7 \quad-1.2$

$119.6 \quad 33.6 \quad-5$.

$197.1 \quad 31.1 \quad-7.0$

$104.7 \quad 36.8 \quad-4.5$

$157.1 \quad 3 . .5 \quad-5.7$

$139.9 \quad 36.3 \quad-2.2$

$122.38 .3 \quad-1.9$

$\begin{array}{rrr}122.1 & 33.3 & -4.8 \\ 95.1 & 36.8 & -3.0\end{array}$

$172.6 \quad 27.3 \quad-7.8$

$238.1 \quad 26.0 \quad-8.9$

$239.7 \quad 11.3 \quad-18.6$

$183.7 \quad 26.2 \quad-10.5$

$187.5 \quad 39.9 \quad-4.9$

$\begin{array}{rrr}238.3 & 21.8 & -12.5 \\ 246.5 & 23.5 & -8.5\end{array}$ 
HELL LOCATION

\begin{tabular}{|c|c|c|}
\hline WNSHIP-RANGE & LONG I TUDE & LATITUDE \\
\hline $25: 18$ d 5 & -49.309 & 37.9483 \\
\hline 253194$] 300 C$ & -39.308 & 37.8276 \\
\hline $25020139 B C 0$ & -99.495 & 37.8355 \\
\hline 2t.sifidesnou & -49.909 & 37.7551 \\
\hline $26.519+1 \angle A H C$ & -99.348 & 97.9093 \\
\hline $26320 \mathrm{~A} 40 \mathrm{CA}$ & -49.509 & 37.8152 \\
\hline ZASL BN2 $313 B C$ & -99.276 & 37.9594 \\
\hline 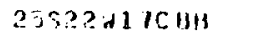 & -99.768 & 37.8774 \\
\hline $25523414 A D O$ & -3ร.Нด7 & 37.8794 \\
\hline $253 \geq 64 \geq 5 C O D$ & -100.128 & 37.8434 \\
\hline $25520 \% 2$ CABt & -100.217 & 37.8563 \\
\hline 2ES29H:20OA & -97.959 & 37.7425 \\
\hline $2652+4: 3 C 13 A$ & -99.950 & 31.7424 \\
\hline $26525 \mathrm{H} 34 \mathrm{BHU}$ & -100.0 .49 & 37.7535 \\
\hline 26526W1 2COAH & -100.118 & 37.8014 \\
\hline $2 \operatorname{cst} 6432100$ & -100.181 & 37.1481 \\
\hline $25535 \times 3 E D C C$ & -100.114 & 37.7405 \\
\hline $215236550 \mathrm{CC}$ & -99.791 & 37.6503 \\
\hline $23324: 4 \quad 43115$ & -99.957 & 37.7369 \\
\hline $2132412613 A A$ & -39.904 & 37.5720 \\
\hline 2132642109.1 & $-100 \cdot 162$ & 37.68171 \\
\hline 24521410000 & -99.594 & 31.6203 \\
\hline $21322 \mathrm{d3} 2 \mathrm{BABS}$ & -49.750 & 37.5739 \\
\hline $24534 \% 9 C \mathrm{HC}$ & -99.957 & 37.6261 \\
\hline 2FOPSU EAIHA & -100.095 & 37.6501 \\
\hline 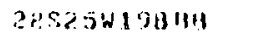 & -100.105 & 31.6058 \\
\hline 2NSTED EAHII & -100.206 & 37.6503 \\
\hline $25920 \%$ EHHH & -99.645 & 37.5592 \\
\hline $25<2.2117040$ & -94.738 & 31.5200 \\
\hline $2532.341 \in C A A$ & -99.839 & 37.5230 \\
\hline $2 C: 29418 B A A$ & -99.987 & 37.5323 \\
\hline acsosd sana & -100.033 & 37.3541 \\
\hline $2 \cos s \omega$ 1Cou & -100.116 & 37.5487 \\
\hline 250204 sentan & -100.123 & 37.1878 \\
\hline$=1: \quad 1: 3 A D C$ & -98.067 & 37.1307 \\
\hline 2389 'd EODIS & -98.340 & 37.1252 \\
\hline $21010+31 \mathrm{HCn}$ & -98.463 & 37.6510 \\
\hline $2 E=9 N$ lUCC & -98.263 & 37.542 .7 \\
\hline 21.318280 & -99.294 & 31.6879 \\
\hline $21013.17313 A$ & -99.426 & 37.8079 \\
\hline aรgow $25 A$ Ho & -99.471 & 37.6163 \\
\hline $28516 ! 2$ 2CCA & -99.046 & 37.6373 \\
\hline $28: 1 / n 2 C$ & -44.153 & 31.6342 \\
\hline $250172150 n+1$ & -99.161 & $37.6,579$ \\
\hline $2+51: 15 \mathrm{CCH}$ & -99.338 & 37.5930 \\
\hline PHSLYH EAAT. & -99.415 & 37.6467 \\
\hline 21317.4313401 & -99.446 & $37.5175_{3}$ \\
\hline $8.451+19313 i)$ & -99.553 & $37.6,013$ \\
\hline$\therefore 13150176 \mathrm{Ce}$ & -99.603 & 38.2186 \\
\hline $21 \% 1 ; 011000$ & $-98.95 A$ & $38 . ? 196$ \\
\hline$=(3151,2 E A 1:)$ & -99.028 & $3 A .2005$ \\
\hline 3:O1OAlOAAC. & -98.253 & 38.1571 \\
\hline 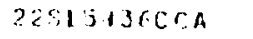 & -96.926 & 34.0900 \\
\hline$\because 213:$ : (140) & -97.613 & $3 H_{0} \cap 344$ \\
\hline $2: 19 ; 11$ cocca & $-131 .+64$ & 34.0509 \\
\hline
\end{tabular}

ACTUAL

DEGRECS C

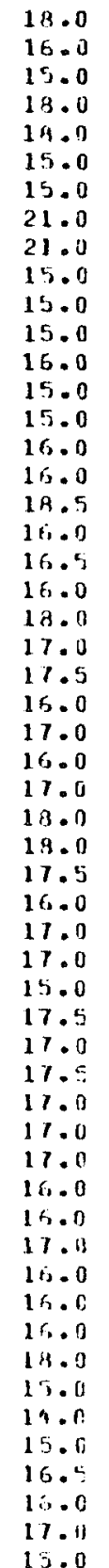

GE O THERMOMETER TEMPERATURES - DEgREES C

QUARTL CHALCEDONY

$M A-K$

220.7

19.0

21.2
36.8

110.1

298.9

242.0
106.5

211.7

176.2

295.3

374.0
343.1

343.1
112.6

143.3

97.1

342.4

276.6

263.6

266.0

196.3
291.6

244.6

243.6

352.6

251.5

255.0

342.4

331.2

308.8

257.

268.0

393.6

$409 . \mathrm{H}$

235.4

87. 0

118.5

144.0
151.5

151.5
303.5

307.3

239.1

316.3

282.5

276.6

259.1

266.2

259.1

272.1
91.1

91.1
89.0

89.0
135.9

90.

222.1

190.7

231.4
19.5

18.7

39.3

23.7

53.8
$5 \div .8$

53.8

42.1

15.7

45.6

98.9

98.9
37.4
35.5

36.0

3E.

32.8

36.4

6.1

28.3
27.2

27.2
22.8
30.1

29.1

31.5

35.8

7.4

33.9
35.9
33.2

33.2

27.8

23.8

33.7

3.6
16.9

16.9
10.5

10.5
11.3

21.6

25.4

14.0

26.8

22.0

23.9

22.5

22.6

25.

27.2
43.7

43.7
10.9

35.

48.

32.2

32.9
NA-K-CA LO

$-13.9$

$-10.4$

-4.4
-15.2

$-12.3$

$-6.4$

$-13.4$

15.5

$-1.2$

1. 2

$-5.4$

3.5

.1

$-3.8$

$-5.3$

$-2.7$

$-9.3$

-7.6
-10.9

$-12.9$

$-8.3$

$-7.1$

$-3.3$

$-2 \cdot 1$

-9.2
-9.5

$-2.6$

$-3.5$

10.5

$-10.5$

-3.1
-30.5
-11.54

$-17$.

$-23.8$

$-23.5$

$-14.3$

$-8.5$

$-1 \% .6$

$-10.2$

$-15.0$

-12.0
-15.0

$-15.0$

$-11$.

-12.3
-9.2

1.5
.6

-2 .

6.3

$-0.0$

$-5.8$ 
HELL LOCATION

\begin{tabular}{|c|c|c|}
\hline $3 H 16-$ & LOMGITUOE & LATITHOE \\
\hline $23015017 A I J C$ & -99.099 & 34.0519 \\
\hline 2f.s1?: 2AA'1 & -98.596 & 37.8246 \\
\hline ers12:1]CC. & $-98, f, t, 2$ & 37.7845 \\
\hline $25: 13315 C A C$ & -96.752 & 87.9011 \\
\hline 2691341530130 & -94.791 & 37.1790 \\
\hline$? 3314 W^{\prime} \quad 1$ & $-7 a . H 03$ & 37.8185 \\
\hline 2ES14K SII!n & -413.882 & 37.4232 \\
\hline 2ESIGULSAAC & -93.1334 & 37.7938 \\
\hline $26.814 .131 \mathrm{ACA}$ & -98.491 & 38.8479 \\
\hline $2 t .515 \mathrm{~W}$ :ISCA & -98.955 & 37.4214 \\
\hline $26515 \mathrm{~N} 13 \mathrm{CCA}$ & -48.918 & 37.7846 \\
\hline $2191141 \mathrm{eOHO}$ & -48.562 & 31.5918 \\
\hline 215134 EAAC & -98.779 & 37.7349 \\
\hline $2 / S 13 d 180011$ & -96.774 & 37.6964 \\
\hline 215154813400 & -48.992 & 31.7202 \\
\hline $215 L 5 \times 25 C C A$ & -98.992 & 37.6668 \\
\hline $2751543 E A D C$ & -98.905 & 37.5578 \\
\hline 2RSA1L QDAN & -98.542 & 37.5 .52 \\
\hline $24 S 13 W 12 H O A$ & $-9 H .694$ & 37.6304 \\
\hline $28 S 14: 300$ & -98.951 & 37.6220 \\
\hline $2 A 515+10 C C A$ & -98.955 & 37.6227 \\
\hline $225 \quad 6 U 17 C C A$ & -93.012 & 38.1329 \\
\hline $2257 \mathrm{~W} 2 \mathrm{C}$ & -98.066 & 38.1627 \\
\hline$z=57+1 / 0 \mathrm{cu}$ & -98.114 & $3 A .1326$ \\
\hline $2 ! 37: 4$ IAPA & -943.039 & 38.0857 \\
\hline $2 ! 591210018$ & -98.312 & 38.0311 \\
\hline $243 \quad 4131 \mathrm{AC}(1)$ & -47.799 & 37.9214 \\
\hline $2455 \% 16 A A$ & -37.869 & 37.9692 \\
\hline $24554 \angle C B \cap n$ & -97.300 & 37.9537 \\
\hline 245 on EOBill & -78.023 & 37.9913 \\
\hline 2155 inla & -97.936 & 31.981 .7 \\
\hline $24.5 \quad \mid W I 3 C \mathrm{CA}$ & $-9 A .04 B$ & 37.95386 \\
\hline $245 \quad 1: 28 A 1$ & -98.075 & 37.9395 \\
\hline $2 A 9,113] C B A$ & -38.139 & $37.918 \mathrm{~F}$ \\
\hline $21519340 \mathrm{AC}$ & $-911.1 \cap 2$ & $37.916,9$ \\
\hline 24591150031 & -98.348 & 37.9439 \\
\hline 24571344 & -98.294 & 37.7232 \\
\hline $215 i 0415 C A 11$ & -18.412 & 37.9619 \\
\hline $2 \cos 10: 150$ & -98.459 & 37.9117 \\
\hline $2 \because 54+14 \mathrm{HAC}$ & -97.733 & 37.81114 \\
\hline 2E: 41 PC.413 & $-1,08.233$ & 37.8497 \\
\hline $2558486(5)$ & -78.341 & 37.8550 \\
\hline$? E S 9426 C C A$ & $-\% A 1.341$ & 37.3569 \\
\hline 2581041111 & -93.950 & $37.87+3$ \\
\hline 255 SU1 SHANH & -97.930 & 37.1956 \\
\hline 2t.3 $6434 \mathrm{illl}$ & -97.771 & 37.74 .75 \\
\hline PESTH IIAA & -78.029 & 37.4343 \\
\hline $25 \% 7 ., 1302111$ & -14.031 & 37.7839 \\
\hline 2Es 1:210100 & -48.096 & 31.7674 \\
\hline 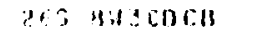 & -48.235 & 57.7547 \\
\hline 265 ynd000n & -48.286 & 37.1947 \\
\hline ?:592:10C11 & -98.345 & 37.7399 \\
\hline $25510 W .5245 A$ & -03.435 & 37.7470 \\
\hline $80 ; 10 \mathrm{~d} 3$ З & -78.376 & $38.26,2$ \\
\hline $215141 \mathrm{All}$ & $-98.13 n$ & 34.2277 \\
\hline
\end{tabular}

ACTUAL TEHP

DEGREES C

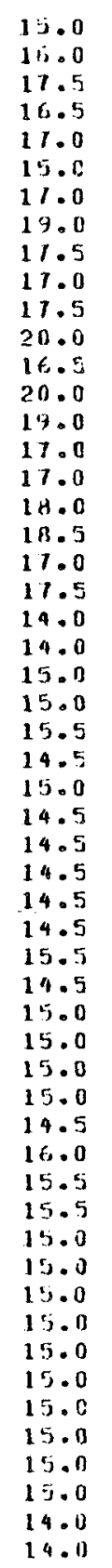

GE OIHERMOMETER TEMPERATURES - DEGACES C

\begin{tabular}{|c|c|c|c|c|}
\hline DUAUYZ & CHALCEDONY & $N A-K$ & $N A-K-C A$ & $N A-K-C A L O$ \\
\hline 61.3 & 33.3 & 160.9 & 35.8 & -2.4 \\
\hline $7: .8$ & 40.1 & 84.9 & 32.8 & -5.6 \\
\hline$r n . t$ & $\exists \in \ldots$ & 94.1 & 31.1 & -3.0 \\
\hline 73.4 & 90.1 & 209.1 & 24.9 & -12.6 \\
\hline 69.0 & 35.1 & 208.8 & 25.1 & -9.3 \\
\hline 72.3 & 38.5 & 224.7 & 24.9 & -8.9 \\
\hline 67.3 & 33.3 & 244.4 & 26.7 & -7.7 \\
\hline 67.3 & 93.3 & 207.1 & 26.0 & -9.2 \\
\hline 75.3 & 43.3 & 351.7 & 26.0 & -9.9 \\
\hline 61.3 & 33.3 & 255.5 & 23.0 & -11.4 \\
\hline $7 \leq .8$ & 40.1 & 338.6 & 17.0 & -13.8 \\
\hline 75.6 & 46.2 & 119.4 & 21.1 & -15.0 \\
\hline 69.0 & $3 \mathrm{s.1}$ & 214.7 & 33.8 & -4.0 \\
\hline $7 \leq .8$ & 40.1 & 115.8 & 33.1 & -4.2 \\
\hline $7 \epsilon .8$ & 43.3 & 295.3 & 24.4 & -10.5 \\
\hline 87.9 & 47.7 & 310.0 & 25.9 & -9.8 \\
\hline 78.2 & 44.8 & 250.9 & 29.5 & -9.3 \\
\hline $7 \in . B$ & 43.3 & 68.2 & 36.5 & -3.3 \\
\hline 75.3 & 41.7 & 183.3 & 19.5 & -18.9 \\
\hline $7 \div .6$ & 46.2 & 132.9 & 35.2 & -1.1 \\
\hline 75.3 & 11.7 & 299.5 & 29.7 & -9.0 \\
\hline 63.7 & 29.5 & 114.2 & 53.9 & 8.8 \\
\hline 67.3 & 33.3 & 14.2 & 30.4 & -12.2 \\
\hline 72.3 & 38.3 & 31.3 & 42.2 & -1.5 \\
\hline 55.9 & 25.4 & 31.4 & 45.9 & 3.1 \\
\hline 76.8 & 93.3 & 67.7 & 43.4 & 2.0 \\
\hline 73.8 & 40.1 & 128.3 & 22.7 & -17.1 \\
\hline 69.0 & 35.1 & 175.6 & 22.7 & -16.3 \\
\hline 73.8 & 40.2 & 112.9 & 18.0 & $-21,1$ \\
\hline 73.8 & 40.1 & 133.7 & 23.1 & -17.5 \\
\hline 72.3 & 30.5 & 110.9 & 31.2 & -4.9 \\
\hline $7 \therefore .8$ & 90.1 & 86.3 & $25 . A$ & -14.6 \\
\hline 70.1 & 35.4 & 118.5 & 10.5 & $-5,9$ \\
\hline 72.3 & 38.5 & 98.8 & 34.2 & -7.2 \\
\hline 76.8 & 43.3 & 179.2 & 48.6 & 2.8 \\
\hline 75.3 & 41.1 & 75.6 & 36.0 & -3.1 \\
\hline 73.6 & 46.2 & 128.5 & 28.2 & -10.8 \\
\hline 76.8 & 93.3 & 172.6 & 30.8 & -10.3 \\
\hline 78.2 & $A 9 . A$ & 80.8 & $3 A .6$ & -2.3 \\
\hline 76.8 & 43.3 & 125.0 & 21.6 & -17.3 \\
\hline $7 \mathrm{~K} . \mathrm{B}$ & 43.3 & 72.1 & 33.3 & -5.5 \\
\hline$t \leq .3$ & 41.7 & 83.6 & 32.6 & -5.5 \\
\hline 75.3 & 91.7 & 85.6 & 42.6 & -.5 \\
\hline 76.8 & 13.3 & 103.1 & 21.7 & -16.1 \\
\hline 72.3 & 38.3 & 117.9 & 17.2 & -1 A. 6 \\
\hline$B 4.8$ & 51.0 & 133.3 & 14.2 & -22.4 \\
\hline 82.3 & 49.1 & $166 . ?$ & 19.6 & -20.1 \\
\hline 8.2.3 & 49.1 & 130.3 & 12.8 & -26.6 \\
\hline 16.4 & 43.5 & 179.1 & 9.3 & -20.9 \\
\hline $7 E .9$ & 43.5 & 115.2 & 17.6 & -18.7 \\
\hline $7 \geq .3$ & 40.1 & 76.7 & 35.5 & -4.4 \\
\hline $75 . f_{.}$ & 16.2 & 106.2 & 15.5 & -10.8 \\
\hline 72.3 & 3 H.S & 152.8 & 2.5 & -27.3 \\
\hline 69.0 & 35.1 & 58.4 & 45.1 & . 6 \\
\hline 79.3 & 19.5 & 121.8 & 3.7 & $-4 \cdot 3$ \\
\hline
\end{tabular}


WELL LOCATION

\begin{tabular}{|c|c|c|}
\hline NIOE & UDE & LATITUT \\
\hline $2151+250$ & -38.111 & .38 .1915 \\
\hline ?1: $94.4 \mathrm{~A}$ & -31.347 & $3 A .2420$ \\
\hline 2199142801811 & -98.311 & 39.1906 \\
\hline $2139431 \mathrm{AAC}$ & -78.348 & 38.1849 \\
\hline ?ISI?H EHAC & -98.669 & 38.2439 \\
\hline 21512416 1 & -98.632 & 38.2392 \\
\hline 21513251380 & -98.798 & 38.2587 \\
\hline $2151+4 \in A$ & -98.899 & 38.2578 \\
\hline Z1S14H lOCn & -98.998 & 38.2 .351 \\
\hline 215144250013 & -48.1105 & 38.1915 \\
\hline$? 151+432 H A C$ & -98.489 & 38.1861 \\
\hline 22511496130 & -98.543 & 38.1581 \\
\hline $2 z 511: 27 A$ & -98.514 & 38.1118 \\
\hline CZS1 3H1 $2 C A C$ & -98.705 & 38.1197 \\
\hline $2.2513: 140 P C$ & $-98 . .392$ & 38.1353 \\
\hline $23013 ; 210011$ & -911.751 & 38.1189 \\
\hline 255134$] \operatorname{CoC} C$ & -98.799 & 37.8431 \\
\hline $2251407 A A C$ & $-98.89 \mathrm{H}$ & 38.1571 \\
\hline$\angle Z S I 4 \sqrt{2} 14 C C A$ & -98.1835 & 38.1335 \\
\hline $225111253 \mathrm{HA}$ & -911.891 & 39.1154 \\
\hline $23511144 C 0 A$ & -98.529 & 38.0746 \\
\hline $2231 \geqslant 422 \mathrm{BCC}$ & -98.636 & 34.0359 \\
\hline $29812.25 \mathrm{CDC}$ & -98.595 & 38.0150 \\
\hline $24: 2 \geq 434 a n:$ & -96.621 & 37.72 .45 \\
\hline $24 S 13 H$ GACH & -98.792 & 37.9955 \\
\hline 245134151340 & -98.794 & 37.9683 \\
\hline 24513615401 & -98.199 & 37.9538 \\
\hline $24 S 13 U 2 \operatorname{SAAI}$ & -38.711 & 37.9393 \\
\hline $21313 \mathrm{H} 31 \mathrm{CCH}$ & -98.1901 & 31.9157 \\
\hline $21314 \mathrm{~W} 5000$ & $-98.8 B 2$ & 37.9702 \\
\hline 213154320160 & -98.994 & 37.9176 \\
\hline $\operatorname{css12} w=C$ & $-48.6,32$ & 37.9014 \\
\hline $25 \$ 12 W 240 D H$ & $-9 B .585$ & 37.8572 \\
\hline 23513 if $30 A 12$ & -31.730 & 37.90 .30 \\
\hline $25013416 \mathrm{EAC}$ & -38.760 & 31.8812 \\
\hline $255134344 A C$ & -98.732 & 37.8317 \\
\hline $2: 514 N 17 C D H$ & -98.1389 & 37.8722 \\
\hline $205142270 \mathrm{AC}$ & -38.0152 & 37.8950 \\
\hline $25515+30 A B C$ & -09.013 & 37.13524 \\
\hline $215,1422 \mathrm{HBH}$ & $-9 A, A 56$ & 31.9557 \\
\hline 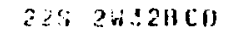 & -97.570 & 38.0755 \\
\hline $2283 \div 20$ & -97.624 & 38.1635 \\
\hline 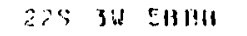 & -97.683 & 38.17 .33 \\
\hline 22031 CAIBA & $-97 \cdot 6,90$ & 38.173 .3 \\
\hline $295 \times 800$ & -47.681 & 3.4 .1505 \\
\hline 225 . SW 5C & -97.661 & 38.1489 \\
\hline $2 ?: 3 A I I A I) C$ & $-1 ; 7.613$ & 39.1535 \\
\hline$z \therefore=3+100$, & -47.8181 & 34.1351 \\
\hline$? \because \quad 1 W 1 \& C A C$ & -97.475 & 58.091 \\
\hline $2 \because 516210 \mathrm{cr}$ & -97.443 & 34.0370 \\
\hline $2951 \mathrm{~W} 34 \mathrm{Cb}$ (3) & -97.425 & $90_{0} 0031$ \\
\hline $2 ! 5 \quad 2040414$ & $-47 . \$ 144$ & 39.0130 \\
\hline 235245004 & $-97 \cdot 2\left(r^{n}\right)$ & 38.0173 \\
\hline$\because \because 2 d \quad 5 B A A$ & -97.547 & $3 B .11714$ \\
\hline $2 \because 2 \quad 2 d 1 \% A B C$ & -91.524 & 39.015 \\
\hline
\end{tabular}

ACIUAL

TEMP

DEGREES C

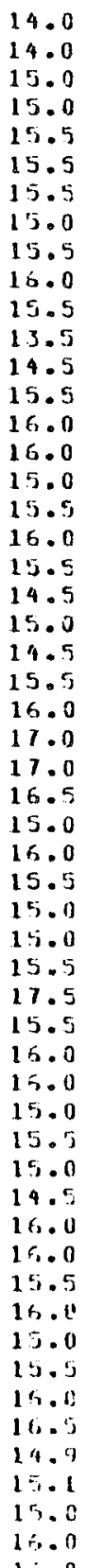

Ge OTHERMOMETER TEMPERATURES - UEgREes C

QUARTZ CHALCEDONY NA-K NA-K-CA NA-K-CALO

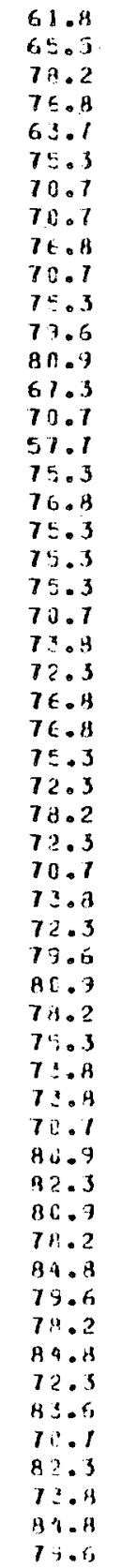

113.8
125.7

96.1

61.6

141.6

130.5

$21 \mathrm{~A} .5$

152.1

146.4

160 .

209.2

180.2

85.9

211.6

260.3

260.3

263.

263.5

201.

235.8

215.3
123.3

131.0

97.3
-187.5

187.5

145.9

66.1

178.3

B0. 6

126.9

217.5

230 .

51.2

123.3

103.9

100.5

198.4

167.5

152.3

184.6

211.5

102.7

119.0

190.9

177.5
220.7

164.0

164.0
123.3

118.1

120.0

59.

67.1

109.2

125.7

105.2

106.2

\begin{tabular}{|c|c|}
\hline $\begin{array}{l}32.1 \\
39.5\end{array}$ & $\begin{array}{l}-8.1 \\
-2.9\end{array}$ \\
\hline 27.9 & $-11 \cdot 2$ \\
\hline $3 \leqslant .4$ & -3.9 \\
\hline 38.5 & $-1 \cdot A$ \\
\hline 93.6 & 9.8 \\
\hline 32.7 & -8.0 \\
\hline 31.1 & -7.1 \\
\hline 24.8 & -10.9 \\
\hline 37.7 & -2.1 \\
\hline 35.5 & -4.1 \\
\hline 18.1 & -10.9 \\
\hline 30.6 & $-2 \cdot 8$ \\
\hline 37.7 & -3.5 \\
\hline 31.0 & -2.9 \\
\hline 32.5 & $-5 . A$ \\
\hline 22.7 & -19.7 \\
\hline 35.9 & -5.1 \\
\hline 33.1 & -5.5 \\
\hline 32.5 & -5.2 \\
\hline 24.4 & -15.1 \\
\hline 29.0 & -9.11 \\
\hline $3 r_{1} \cdot 4$ & -4.5 \\
\hline 47.4 & 5.4 \\
\hline 32.0 & $-E .6$ \\
\hline 29.9 & -1.4 \\
\hline 26.7 & -12.0 \\
\hline 39.5 & of \\
\hline 23.8 & -14.1 \\
\hline 35.5 & -1.5 \\
\hline 33.0 & -5.0 \\
\hline 15.8 & 3.2 \\
\hline 23.7 & -13.4 \\
\hline 34.1 & -3.0 \\
\hline 42.2 & 2.9 \\
\hline 27.6 & -11.1 \\
\hline 29.8 & -6.5 \\
\hline 36.8 & -.7 \\
\hline 31.0 & $-6 \cdot 3$ \\
\hline 34.9 & -4.9 \\
\hline 10.8 & -27.5 \\
\hline 11.1 & -25.8 \\
\hline 23.1 & -18.0 \\
\hline 21.9 & -19.4 \\
\hline 27.1 & -15.3 \\
\hline 17.5 & -20.1 \\
\hline 9.6 & -25.2 \\
\hline 49.9 & -1.0 \\
\hline 3.9 & -29.3 \\
\hline 9.6 & -25.9 \\
\hline 9.9 & -28.1 \\
\hline 9.5 & -26.1 \\
\hline 22.9 & -9.9 \\
\hline 10.3 & -23.8 \\
\hline 5.1 & -27.1 \\
\hline
\end{tabular}


WELL LOCAIION

IOHNSHIF-RANGE LONGITUDC LATITUDE

\begin{tabular}{|c|c|}
\hline$\$ 1.496$ & 38.018 \\
\hline 97.567 & 38.0265 \\
\hline-97.6 .50 & 38.0464 \\
\hline-47.650 & 38.0374 \\
\hline-97.634 & $38.033 \mathrm{~A}$ \\
\hline-91.599 & 38.0121 \\
\hline$-97.44 A$ & 37.9152 \\
\hline-97.425 & 37.961 \\
\hline-97.418 & 37.958 \\
\hline-97.462 & 37.9381 \\
\hline-97.556 & $31.9 A B$ \\
\hline-47.579 & 31.9902 \\
\hline$-97.47 \mathrm{~A}$ & 37.9716 \\
\hline-97.565 & 37.962 \\
\hline-97.517 & 37.953 \\
\hline-97.512 & 31.9490 \\
\hline-97.532 & 37.931 \\
\hline$-97.55 A$ & 37.930 \\
\hline-97.673 & 37.993 \\
\hline-98.67 .3 & 37.977 \\
\hline-97.634 & 37.957 \\
\hline-97.680 & 37.953 \\
\hline-97.046 & 37.948 \\
\hline-97.605 & 37.945 \\
\hline-47.624 & 37.939 \\
\hline-97.679 & 37.921 \\
\hline-47.592 & 37.926 \\
\hline-97.101 & 38.447 \\
\hline-97.666 & 38.435 \\
\hline-97.778 & 38.959 \\
\hline-47.451 & 39.351 \\
\hline-97.680 & $3 H .403$ \\
\hline-47.598 & 38.362 \\
\hline-97.737 & 38.400 \\
\hline-7.125 & 39.404 \\
\hline-97.740 & $3 n .511$ \\
\hline-97.426 & 38.304 \\
\hline-97.700 & 33.301 \\
\hline-91.143 & $38.3 ? 4$ \\
\hline-97.108 & 39.331 \\
\hline-41.726 & 38.303 \\
\hline-37.623 & 38.258 \\
\hline-97.699 & 39.242 \\
\hline-37.561 & 38.243 \\
\hline-97.677 & $3 H .215$ \\
\hline-97.676 & 34.200 \\
\hline$-71.6,83$ & .38 .133 \\
\hline-97.571 & 3.174 \\
\hline-47.668 & 38.145 \\
\hline-97.618 & $3 B .1 A 2$ \\
\hline-97.707 & $39.2 ? 1$ \\
\hline-97.123 & $38.19 ! 1$ \\
\hline-47.714 & 38.112 \\
\hline-97 & 58.127 \\
\hline & \\
\hline
\end{tabular}

ACTUAL

DEGREES C

15.8
15.9
16.1
16.0
16.3
15.2
17.5
15.1
15.3
15.4
15.9
15.0
15.1
14.8
15.1
14.8
15.0
15.6
14.5
14.0
14.0
11.0
11.2
19.8
19.8
15.0
14.5
16.0
15.1
15.8
14.5
15.1
15.0
15.8
15.6
15.6
14.4
15.4
15.0
15.0
15.0
15.0
15.3
15.0
15.0
16.0
15.3
15.5
13.5
11.13
16.0
16.0
15.5
15.0
17.0

GE OTHERMOMETER TEMPERATURES - DEGREES C

DUARTZ CHALCCUONY NA-X NA-K-CA NA-K-CA LI

\begin{tabular}{|c|c|}
\hline 75.3 & 41.1 \\
\hline $7: A$ & 90.1 \\
\hline 70.7 & 36.8 \\
\hline 70.7 & 36.8 \\
\hline 76.8 & 43.3 \\
\hline 72.3 & 38.5 \\
\hline 94.0 & 51.5 \\
\hline 82.3 & 45.1 \\
\hline 63.0 & 35.1 \\
\hline 69.0 & 35.1 \\
\hline 73. A & 40.1 \\
\hline 69.0 & 35.1 \\
\hline 63.0 & 35.1 \\
\hline 62.7 & 29.5 \\
\hline 65.5 & 31.4 \\
\hline $6 \therefore .7$ & 29.5 \\
\hline 12.3 & 38.0 \\
\hline $6 \% .5$ & 23.3 \\
\hline 55.5 & 21.93 \\
\hline $59 . A$ & 25.4 \\
\hline$S S . A$ & 25.4 \\
\hline 51.7 & $23 . ?$ \\
\hline 59.8 & 25.9 \\
\hline $4=-5$ & 10.7 \\
\hline 55.5 & 21.0 \\
\hline $6: .7$ & 29.5 \\
\hline 57.7 & 23.2 \\
\hline 90.7 & 58.1 \\
\hline 90.7 & 58.1 \\
\hline 73.8 & 10.1 \\
\hline 79.6 & 46.2 \\
\hline 90.7 & 58.1 \\
\hline 83.6 & 50.5 \\
\hline 70.7 & 36.8 \\
\hline 75.6 & 46.2 \\
\hline 67.3 & 3.3 .3 \\
\hline 78.2 & 44.8 \\
\hline 80.9 & 47.7 \\
\hline 67.3 & 33.3 \\
\hline ค0. & 97.7 \\
\hline 76.8 & 43.3 \\
\hline 85.5 & 55.7 \\
\hline 78.2 & 44.18 \\
\hline 78.2 & $14 . \mu$ \\
\hline 7503 & 11.1 \\
\hline 75.5 & $4 E .2$ \\
\hline $1 \therefore .8$ & 40.1 \\
\hline 72.3 & '3.9. \\
\hline $7 \because .8$ & 40.1 \\
\hline 76.8 & 43.3 \\
\hline 76.8 & 43.3 \\
\hline 92.3 & 49.1 \\
\hline 82.3 & 47.1 \\
\hline 83.6 & 50.5 \\
\hline $5 \% .8$ & .25 .4 \\
\hline
\end{tabular}

\begin{tabular}{|c|c|c|}
\hline 136.0 & 5.3 & -26.7 \\
\hline 149.1 & 30.3 & -1.7 \\
\hline 115.8 & 29.7 & -11.2 \\
\hline 198.1 & 45.7 & 3.0 \\
\hline 155.2 & 31.2 & -9.1 \\
\hline 187.5 & 33.5 & $-8 \cdot B$ \\
\hline 168.7 & 3.8 & -32.1 \\
\hline 87.3 & 7.5 & $-27 \cdot 6$ \\
\hline 114.7 & 30.7 & -9.8 \\
\hline 98.1 & 38.0 & -5.2 \\
\hline 115.8 & 31.5 & -8.4 \\
\hline 167.5 & 31.9 & -4.6 \\
\hline 112.2 & 30.8 & -11.3 \\
\hline 115.1 & 38.4 & -5.8 \\
\hline 173.9 & 32.4 & -10.9 \\
\hline $196 \cdot 6$ & 36.5 & -7.0 \\
\hline 117.4 & 32.4 & -10.1 \\
\hline 114.6 & 35.5 & -4.1 \\
\hline 127.5 & 40.1 & 1.0 \\
\hline 107.1 & 38.7 & -3.4 \\
\hline 128.0 & 43.1 & -3.5 \\
\hline 111.6 & 39.3 & -3.5 \\
\hline 105.1 & 41.5 & .3 \\
\hline 123.3 & 30.2 & -5.8 \\
\hline 110.8 & 34.0 & -3.8 \\
\hline 85.9 & 94.7 & 1.1 \\
\hline 103.0 & 31.3 & -8.8 \\
\hline $202 \cdot 1$ & 17.1 & -22.0 \\
\hline 132.2 & 26.0 & -19.1 \\
\hline 204.7 & 12.8 & -23.9 \\
\hline 12.4 .8 & 21.1 & -19.7 \\
\hline $203 \cdot 1$ & 15.2 & -21.5 \\
\hline 187.5 & 7.1 & -26.7 \\
\hline 159.6 & 15.2 & -23.2 \\
\hline 214.1 & 16.4 & -20.4 \\
\hline 151.3 & 23.5 & -19.2 \\
\hline 100.9 & 9.5 & -27.4 \\
\hline 225.3 & 17.7 & -19.3 \\
\hline 162.1 & 23.1 & -18.5 \\
\hline 199.6 & 18.5 & $-13 . ?$ \\
\hline 202.4 & 20.1 & -18.4 \\
\hline $155 \cdot 6$ & 9.8 & $-2 \varepsilon_{0} ?$ \\
\hline 202.4 & 21.3 & -15.0 \\
\hline 113.5 & 35.9 & $-h .0$ \\
\hline 170.2 & 17.0 & -19.4 \\
\hline 198.8 & 25.0 & -14.5 \\
\hline 178.3 & 22.6 & -17.5 \\
\hline 194.4 & 30.7 & -12.5 \\
\hline 170.5 & 23.7 & -16.4 \\
\hline 112.6 & 12.2 & -23.8 \\
\hline 177.5 & 22.3 & -16.7 \\
\hline 203.5 & 21.0 & -16.8 \\
\hline 199.2 & 21.3 & $-19 . t$ \\
\hline 114.6 & 31.4 & $-12 \cdot 0$ \\
\hline 96.1 & 51.5 & 5.2 \\
\hline
\end{tabular}




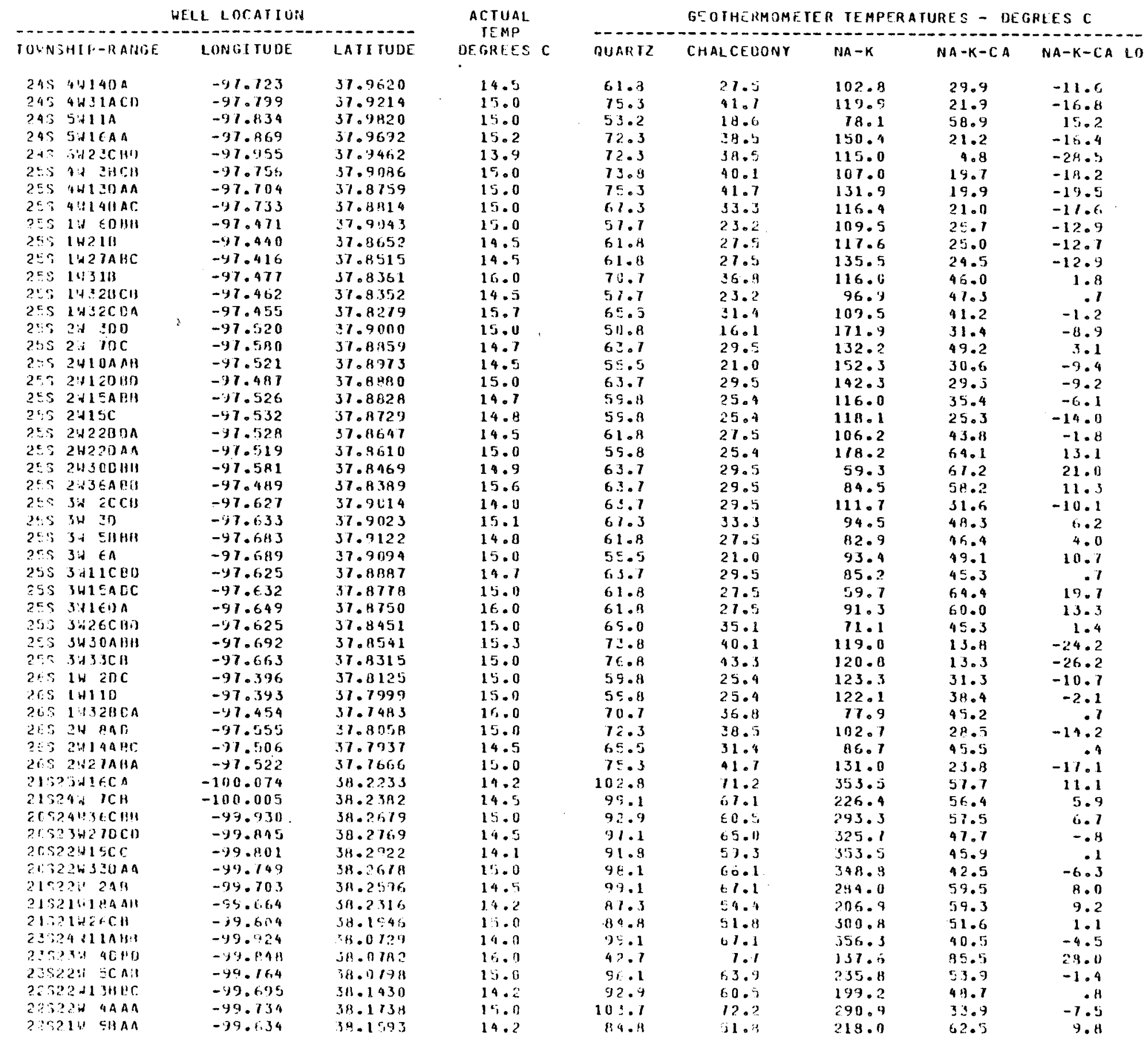




\begin{tabular}{|c|c|c|c|c|c|c|c|c|}
\hline \multicolumn{3}{|c|}{ WELL LOCATION } & $\begin{array}{l}\text { ACTUAL } \\
\text { TEMP }\end{array}$ & \multicolumn{5}{|c|}{ GEOTHERMOMETER TEMPERATURES - DEGAEES C } \\
\hline TOANSHIF-RANEE & LONGI TUDE & LAIITUDE & DEGREES C & QUARTL & CHALCEOONY & $N A-K$ & $N A-K-C A$ & NA-K-CA LO \\
\hline $215 \geq 011254$ & -99.547 & 38.2000 & 14.5 & 90.7 & 58.1 & 279.7 & 50.8 & 1.8 \\
\hline $21520 \div 1300$ & -99.467 & 34.2216 & 15.0 & 20.7 & 58.1 & 276.5 & 19.7 & $-2 B .7$ \\
\hline $215 \sin 360 \operatorname{la}$ & -99.575 & 30.1810 & 14.5 & B१. $A$ & $\leq 1.8$ & 247.1 & 35.9 & -8.1 \\
\hline $21 S 19 \backsim 2 E 4 \mathrm{CA}$ & -59.370 & 34.1995 & 15.0 & 50.9 & 16.1 & 177.2 & 59.4 & 1.0 \\
\hline 21SIBH3 3 BCB & -99.313 & 38.1948 & 14.0 & 81.3 & 54.4 & 251.7 & 33.7 & -9.5 \\
\hline 2शSL & -99.311 & 38.1576 & 14.3 & $7 E .8$ & 43.5 & 126.9 & 61.9 & 11.0 \\
\hline $27519+114418$ & -99.267 & 38.1573 & 14.3 & $7: .2$ & 94.18 & 148.0 & $5 E .4$ & 6.4 \\
\hline 2.S1IHIJABE & -99.217 & 38.1426 & 15.0 & $6 \subseteq .7$ & 29.3 & 120.9 & 45.7 & 3.8 \\
\hline $21917: 311109$ & -99.233 & $3 H .1361$ & 14.2 & 75.3 & 11.7 & 179.1 & 34.1 & -8.7 \\
\hline $22511: 4 B H$ & -99.203 & 38.1154 & 15.0 & 89.6 & 56.9 & 147.9 & 62.5 & 10.9 \\
\hline $20,15 \mathrm{~W} \in \mathbb{R}$ & -99.127 & 38.1707 & 13.8 & 65.5 & 31.4 & 92.7 & 49.8 & -3.3 \\
\hline $1351 \sin 2000$ & -98.722 & 38.3544 & 14.2 & 59.8 & 25.4 & 126.6 & 19.3 & 3.5 \\
\hline $18 S 144 \leq 515$ & -96.832 & 38.4943 & 15.2 & 81.3 & 54.4 & 201.4 & 38.5 & -6.9 \\
\hline $19 S 19,61311$ & -38.919 & 38.4336 & 14.2 & $7 E \cdot 8$ & 43.3 & 122.2 & 42.5 & $-7 . c$ \\
\hline IPS154: 29111 & -48.993 & $34.96,26$ & 14.2 & 85.0 & 53.1 & 202.4 & 34.5 & $-12 \cdot 0$ \\
\hline 19516!11401! & -99.053 & 38.9808 & 14.3 & 84.8 & 51.8 & 220.7 & 10.8 & -7.4 \\
\hline 1Psildacon & -99.154 & 38.4911 & 14.0 & 79.6 & 46.2 & 171.1 & 16.5 & -2.6 \\
\hline 1RS1BA27CC & -99.305 & 38.4522 & 14.2 & 80.9 & 47.1 & 183.3 & 59.9 & 6.5 \\
\hline $1 P=\{A 42 S C 1\}$ & -99.268 & 38.4556 & 14.0 & 8.3 .6 & 50.5 & 211.0 & 49.3 & -5.7 \\
\hline $1091 / 4254 A$ & -99.218 & 30.1529 & 14.2 & $7 \varepsilon .8$ & 43.3 & 199.8 & 36.9 & -10.1 \\
\hline 1FSLOU $3000 \mathrm{C}$ & $-99.34 A$ & $3 A .4514$ & 14.2 & 80.9 & 47.1 & 194.4 & 39.2 & -8.6 \\
\hline 1मSIMU2TAAA & -99.401 & 38.4639 & 14.0 & B..8 & 51.8 & 249.3 & 36.7 & -9.6 \\
\hline IESIGULACCr. & -99.472 & 38.4199 & 14.2 & 96.1 & 66.1 & 246.1 & 42.8 & -6.1 \\
\hline 195204170CC & -99.556 & 38.4796 & 14.0 & $9 \div .0$ & $\epsilon 2.8$ & 233.6 & 38.8 & -4.9 \\
\hline 1F:22UउGAH & -49.701 & 38.4480 & 14.3 & 102.8 & 11.2 & 256.6 & 51.0 & 3.3 \\
\hline 1452343000 & -99.838 & $3 R_{0} 4515$ & 15.0 & 92.9 & 60.5 & 349.6 & 63.1 & 13.9 \\
\hline 1FS24W2SBHA & -99.930 & 38.4633 & 14.0 & 100.0 & 68.2 & 265.7 & 45.1 & -1.5 \\
\hline $119 \$ 24: 1226 C$ & -59.967 & 38.4661 & 14.0 & 85.6 & 56.9 & 220.1 & 65.7 & 13.2 \\
\hline IFSIT'150AB & -99.187 & 38.4156 & 14.5 & 79.6 & 46.2 & 234.5 & 56.0 & 3.8 \\
\hline D!nITHIJCBC & -99.159 & 38.4838 & 14.3 & 78.2 & 14.8 & 199.2 & 41.0 & -1.5 \\
\hline I8sisid 7Co & -99.126 & 38.4956 & 14.0 & 98.1 & $E G .1$ & 205.0 & 49.1 & -1.2 \\
\hline IHS1GH22BMU & -99.086 & 38.4782 & 14.0 & $7 E . B$ & 43.3 & 217.5 & 46.3 & .1 \\
\hline $1451501300 \mathrm{C}$ & -99.021 & 38.4517 & 14.0 & 85.6 & 56.9 & 227.8 & 30.1 & -11.6 \\
\hline $1 R 915112306$ & $-9 B .956$ & 38.4662 & 15.0 & 98.1 & 66.1 & 269.2 & 28.4 & -13.5 \\
\hline 105194220 & -98.899 & 38.4462 & 14.0 & 91.8 & 59.3 & 226.3 & 35.7 & -7.5 \\
\hline IGS14U2OAAII & -98.888 & 38.3910 & 15.0 & 73.8 & 90.1 & 93.4 & 38.8 & -5.9 \\
\hline ZCS19N PHBO & -98.900 & 38.3312 & 14.3 & 75.3 & 41.7 & 135.4 & 45.7 & -2.9 \\
\hline 2R31541 $3 A 00$ & -78.922 & 38.3131 & 14.0 & $7 n .2$ & 44.4 & 152.7 & 46.7 & -.6 \\
\hline 15s13:1 oc & -98.170 & 38.4078 & 14.0 & 16.8 & 43.5 & 150.9 & 45.4 & -2.1 \\
\hline
\end{tabular}

